\title{
SUPPORTING INFORMATION Benchmarking Hybrid Atomistic/Coarse-Grained Schemes for Proteins with an Atomistic Water Layer
}

\author{
Annick Renevey, Sereina Riniker* \\ [*] Laboratory of Physical Chemistry, ETH Zurich, Vladimir-Prelog-Weg 2, 8093 Zurich, Switzerland \\ Email: sriniker@ethz.ch
}

\section{Contents}

1 Additional Tables $\quad$ S2

2 Summary: Analyses Proteins $\quad \mathbf{S 1 2}$

2.1 Protein 1aki . . . . . . . . . . . . . . . . . . . . . . . . S12

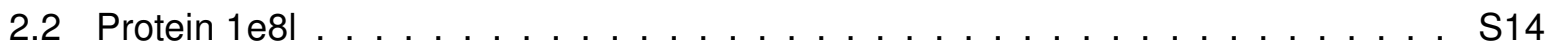

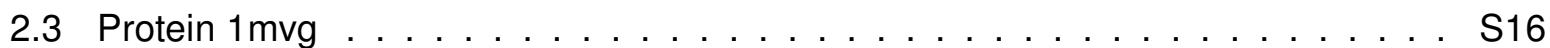

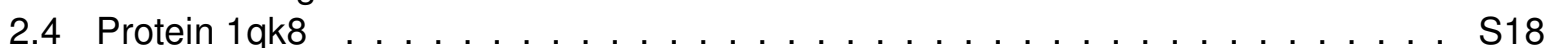

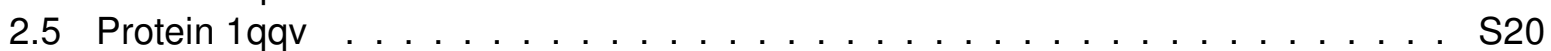

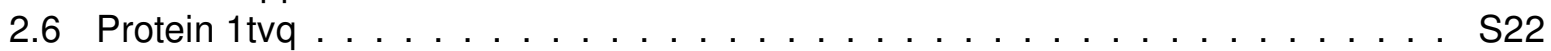

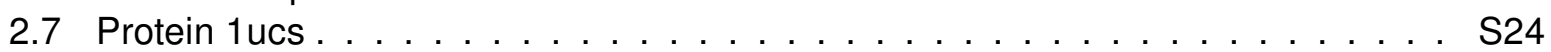

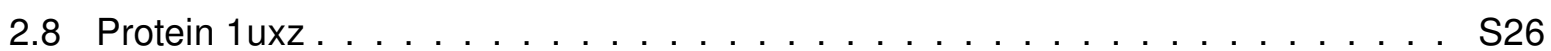

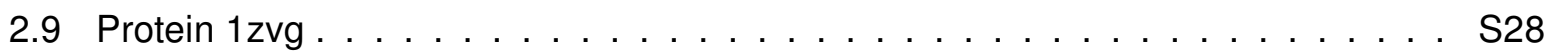

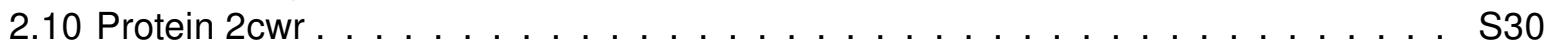

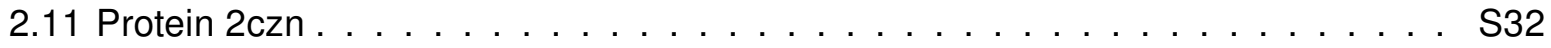

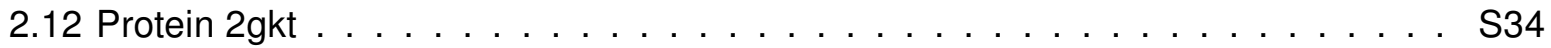

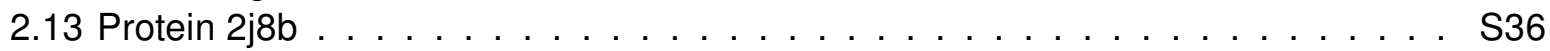

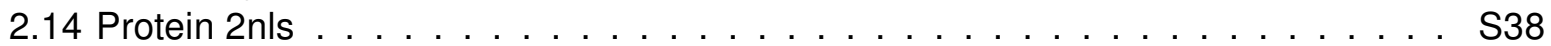

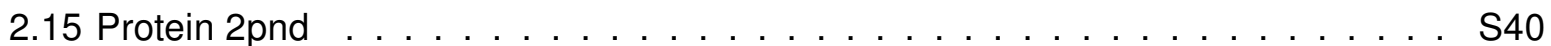

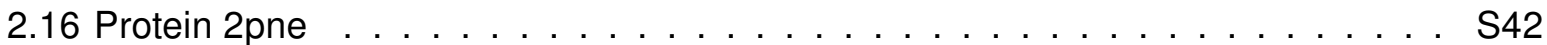

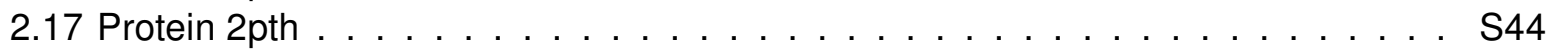

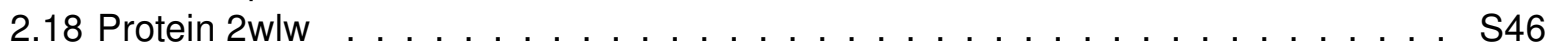

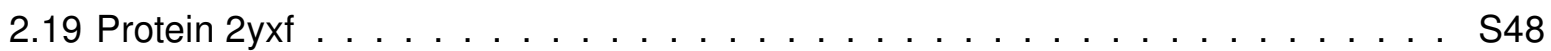

2.20 Protein $3 \mathrm{ca} 7 \ldots \ldots \ldots \ldots \ldots \ldots \ldots \ldots \ldots$

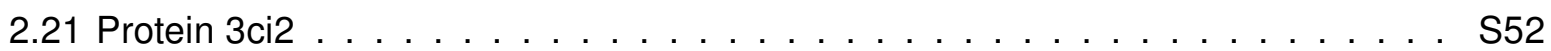

2.22 Protein 3 eye $\ldots \ldots \ldots \ldots \ldots \ldots \ldots$. . . . . . . . . . . . . . . . . . . . . . . . . . . . . .

3 Additional Figures $\quad$ S56 


\section{Additional Tables}

Table S1: Average backbone atom-positional root-mean-square deviation (RMSD) over the last $5 \mathrm{~ns}$ of simulation for the 3-4 replicas and averaged over the replicas. For the individual replicas, the uncertainty estimate is the standard deviation over the frames in the $5 \mathrm{~ns}$. For the average over replicas, the uncertainty estimate is the standard deviation over the averages of the individual replicas.

\begin{tabular}{|c|c|c|c|c|c|c|}
\hline Protein & Method & Rep. 1 & Rep. 2 & Rep. 3 & Rep. 4 & Average \\
\hline \multirow[t]{3}{*}{ 1aki } & Full AT & $0.279 \pm 0.010$ & $0.308 \pm 0.012$ & $0.244 \pm 0.017$ & $0.222 \pm 0.012$ & $0.263 \pm 0.033$ \\
\hline & Full CG & $0.391 \pm 0.019$ & $0.235 \pm 0.010$ & $0.412 \pm 0.009$ & & $0.346 \pm 0.079$ \\
\hline & Layer Sch. 2 & $0.224 \pm 0.009$ & $0.230 \pm 0.010$ & $0.241 \pm 0.009$ & & $0.232 \pm 0.007$ \\
\hline \multirow[t]{3}{*}{$1 \mathrm{e} 8 \mathrm{I}$} & Full AT & $0.276 \pm 0.011$ & $0.325 \pm 0.015$ & $0.265 \pm 0.010$ & $0.402 \pm 0.017$ & $0.319 \pm 0.054$ \\
\hline & Full CG & $0.366 \pm 0.011$ & $0.279 \pm 0.007$ & $0.244 \pm 0.014$ & & $0.296 \pm 0.052$ \\
\hline & Layer Sch. 2 & $0.252 \pm 0.010$ & $0.217 \pm 0.011$ & $0.229 \pm 0.014$ & & $0.233 \pm 0.015$ \\
\hline \multirow[t]{3}{*}{$1 \mathrm{mvg}$} & Full AT & $0.328 \pm 0.012$ & $0.281 \pm 0.011$ & $0.564 \pm 0.025$ & $0.301 \pm 0.030$ & $0.369 \pm 0.114$ \\
\hline & Full CG & $0.377 \pm 0.009$ & $0.301 \pm 0.012$ & $0.247 \pm 0.009$ & & $0.308 \pm 0.054$ \\
\hline & Layer Sch. 2 & $0.232 \pm 0.013$ & $0.222 \pm 0.013$ & $0.197 \pm 0.010$ & & $0.217 \pm 0.015$ \\
\hline \multirow[t]{3}{*}{$1 \mathrm{qk} 8$} & Full AT & $0.225 \pm 0.009$ & $0.453 \pm 0.011$ & $0.270 \pm 0.012$ & $0.276 \pm 0.020$ & $0.306 \pm 0.087$ \\
\hline & Full CG & $0.283 \pm 0.019$ & $0.383 \pm 0.009$ & $0.277 \pm 0.009$ & & $0.314 \pm 0.049$ \\
\hline & Layer Sch. 2 & $0.308 \pm 0.013$ & $0.256 \pm 0.009$ & $0.275 \pm 0.009$ & & $0.280 \pm 0.022$ \\
\hline \multirow[t]{3}{*}{ 1qqv } & Full AT & $0.506 \pm 0.035$ & $0.763 \pm 0.017$ & $0.774 \pm 0.015$ & $0.722 \pm 0.017$ & $0.691 \pm 0.109$ \\
\hline & Full CG & $0.558 \pm 0.042$ & $0.791 \pm 0.034$ & $0.798 \pm 0.033$ & & $0.716 \pm 0.112$ \\
\hline & Layer Sch. 2 & $0.280 \pm 0.021$ & $0.393 \pm 0.025$ & $0.304 \pm 0.030$ & & $0.326 \pm 0.049$ \\
\hline \multirow[t]{3}{*}{1 tvq } & Full AT & $0.243 \pm 0.017$ & $0.368 \pm 0.012$ & $0.269 \pm 0.023$ & $0.257 \pm 0.009$ & $0.285 \pm 0.049$ \\
\hline & Full CG & $0.239 \pm 0.010$ & $0.228 \pm 0.010$ & $0.295 \pm 0.009$ & & $0.254 \pm 0.030$ \\
\hline & Layer Sch. 2 & $0.272 \pm 0.023$ & $0.302 \pm 0.017$ & $0.262 \pm 0.021$ & & $0.278 \pm 0.017$ \\
\hline \multirow[t]{3}{*}{1 ucs } & Full AT & $0.094 \pm 0.012$ & $0.148 \pm 0.016$ & $0.235 \pm 0.011$ & $0.265 \pm 0.012$ & $0.185 \pm 0.068$ \\
\hline & Full CG & $0.124 \pm 0.009$ & $0.167 \pm 0.011$ & $0.250 \pm 0.009$ & & $0.180 \pm 0.052$ \\
\hline & Layer Sch. 2 & $0.123 \pm 0.009$ & $0.216 \pm 0.011$ & $0.221 \pm 0.012$ & & $0.186 \pm 0.045$ \\
\hline \multirow[t]{3}{*}{$1 \mathrm{uxz}$} & Full AT & $0.184 \pm 0.020$ & $0.242 \pm 0.015$ & $0.217 \pm 0.015$ & $0.253 \pm 0.013$ & $0.224 \pm 0.027$ \\
\hline & Full CG & $0.235 \pm 0.016$ & $0.294 \pm 0.031$ & $0.163 \pm 0.009$ & & $0.231 \pm 0.053$ \\
\hline & Layer Sch. 2 & $0.174 \pm 0.010$ & $0.158 \pm 0.007$ & $0.151 \pm 0.009$ & & $0.161 \pm 0.010$ \\
\hline \multirow[t]{3}{*}{$1 z v g$} & Full AT & $0.199 \pm 0.011$ & $0.194 \pm 0.011$ & $0.121 \pm 0.010$ & $0.190 \pm 0.023$ & $0.176 \pm 0.032$ \\
\hline & Full CG & $0.184 \pm 0.010$ & $0.181 \pm 0.016$ & $0.239 \pm 0.023$ & & $0.201 \pm 0.026$ \\
\hline & Layer Sch. 2 & $0.147 \pm 0.012$ & $0.151 \pm 0.011$ & $0.239 \pm 0.018$ & & $0.179 \pm 0.042$ \\
\hline \multirow[t]{3}{*}{$2 c w r$} & Full AT & $0.120 \pm 0.010$ & $0.225 \pm 0.031$ & $0.440 \pm 0.010$ & $0.365 \pm 0.011$ & $0.287 \pm 0.124$ \\
\hline & Full CG & $0.264 \pm 0.015$ & $0.226 \pm 0.011$ & $0.239 \pm 0.010$ & & $0.243 \pm 0.016$ \\
\hline & Layer Sch. 2 & $0.223 \pm 0.010$ & $0.139 \pm 0.014$ & $0.140 \pm 0.009$ & & $0.167 \pm 0.039$ \\
\hline \multirow[t]{3}{*}{$2 c z n$} & Full AT & $0.394 \pm 0.009$ & $0.547 \pm 0.017$ & $0.379 \pm 0.008$ & $0.363 \pm 0.008$ & $0.421 \pm 0.074$ \\
\hline & Full CG & $0.386 \pm 0.010$ & $0.473 \pm 0.010$ & $0.461 \pm 0.010$ & & $0.440 \pm 0.039$ \\
\hline & Layer Sch. 2 & $0.298 \pm 0.007$ & $0.291 \pm 0.011$ & $0.286 \pm 0.009$ & & $0.291 \pm 0.005$ \\
\hline \multirow[t]{3}{*}{$2 \mathrm{gkt}$} & Full AT & $0.477 \pm 0.016$ & $0.407 \pm 0.023$ & $0.216 \pm 0.020$ & $0.457 \pm 0.019$ & $0.389 \pm 0.103$ \\
\hline & Full CG & $0.344 \pm 0.014$ & $0.301 \pm 0.015$ & $0.251 \pm 0.023$ & & $0.299 \pm 0.038$ \\
\hline & Layer Sch. 2 & $0.367 \pm 0.013$ & $0.447 \pm 0.018$ & $0.254 \pm 0.012$ & & $0.356 \pm 0.079$ \\
\hline \multirow[t]{3}{*}{$2 \mathrm{j} 8 \mathrm{~b}$} & Full AT & $0.187 \pm 0.030$ & $0.370 \pm 0.038$ & $0.310 \pm 0.010$ & $0.261 \pm 0.013$ & $0.282 \pm 0.067$ \\
\hline & Full CG & $0.346 \pm 0.017$ & $0.310 \pm 0.019$ & $0.430 \pm 0.024$ & & $0.362 \pm 0.050$ \\
\hline & Layer Sch. 2 & $0.400 \pm 0.015$ & $0.288 \pm 0.018$ & $0.213 \pm 0.018$ & & $0.300 \pm 0.077$ \\
\hline \multirow[t]{3}{*}{ 2nls } & Full AT & $0.250 \pm 0.018$ & $0.170 \pm 0.018$ & $0.417 \pm 0.009$ & $0.153 \pm 0.017$ & $0.248 \pm 0.104$ \\
\hline & Full CG & $0.178 \pm 0.011$ & $0.187 \pm 0.018$ & $0.290 \pm 0.013$ & & $0.218 \pm 0.051$ \\
\hline & Layer Sch. 2 & $0.168 \pm 0.011$ & $0.176 \pm 0.010$ & $0.159 \pm 0.026$ & & $0.168 \pm 0.007$ \\
\hline \multirow[t]{3}{*}{ 2pnd } & Full AT & $0.198 \pm 0.012$ & $0.204 \pm 0.013$ & $0.175 \pm 0.013$ & $0.196 \pm 0.012$ & $0.193 \pm 0.011$ \\
\hline & Full CG & $0.204 \pm 0.008$ & $0.236 \pm 0.011$ & $0.222 \pm 0.010$ & & $0.221 \pm 0.013$ \\
\hline & Layer Sch. 2 & $0.159 \pm 0.008$ & $0.159 \pm 0.010$ & $0.169 \pm 0.009$ & & $0.162 \pm 0.005$ \\
\hline
\end{tabular}




\begin{tabular}{lllllll}
\hline 2pne & Full AT & $0.133 \pm 0.020$ & $0.127 \pm 0.022$ & $0.173 \pm 0.017$ & $0.133 \pm 0.020$ & $0.141 \pm 0.018$ \\
& Full CG & $0.239 \pm 0.019$ & $0.235 \pm 0.019$ & $0.283 \pm 0.019$ & & $0.252 \pm 0.022$ \\
& Layer Sch. 2 & $0.151 \pm 0.015$ & $0.149 \pm 0.017$ & $0.157 \pm 0.011$ & & $0.152 \pm 0.003$ \\
\hline 2pth & Full AT & $0.233 \pm 0.010$ & $0.257 \pm 0.015$ & $0.387 \pm 0.009$ & $0.236 \pm 0.011$ & $0.278 \pm 0.063$ \\
& Full CG & $0.424 \pm 0.024$ & $0.487 \pm 0.016$ & $0.732 \pm 0.039$ & & $0.548 \pm 0.133$ \\
& Layer Sch. 2 & $0.251 \pm 0.011$ & $0.241 \pm 0.016$ & $0.204 \pm 0.017$ & & $0.232 \pm 0.020$ \\
\hline 2wlw & Full AT & $0.301 \pm 0.016$ & $0.216 \pm 0.010$ & $0.211 \pm 0.008$ & $0.189 \pm 0.011$ & $0.229 \pm 0.042$ \\
& Full CG & $0.306 \pm 0.008$ & $0.318 \pm 0.008$ & $0.323 \pm 0.008$ & & $0.319 \pm 0.011$ \\
& Layer Sch. 2 & $0.224 \pm 0.008$ & $0.166 \pm 0.007$ & $0.226 \pm 0.012$ & & $0.205 \pm 0.028$ \\
\hline 2yxf & Full AT & $0.148 \pm 0.010$ & $0.208 \pm 0.020$ & $0.284 \pm 0.013$ & $0.162 \pm 0.019$ & $0.200 \pm 0.053$ \\
& Full CG & $0.230 \pm 0.016$ & $0.296 \pm 0.010$ & $0.357 \pm 0.018$ & & $0.294 \pm 0.052$ \\
& Layer Sch. 2 & $0.230 \pm 0.022$ & $0.162 \pm 0.014$ & $0.176 \pm 0.014$ & & $0.189 \pm 0.029$ \\
\hline 3ca7 & Full AT & $0.187 \pm 0.025$ & $0.226 \pm 0.017$ & $0.291 \pm 0.039$ & $0.343 \pm 0.060$ & $0.262 \pm 0.060$ \\
& Full CG & $0.209 \pm 0.012$ & $0.262 \pm 0.035$ & $0.189 \pm 0.011$ & & $0.220 \pm 0.031$ \\
& Layer Sch. 2 & $0.450 \pm 0.034$ & $0.646 \pm 0.010$ & $0.460 \pm 0.022$ & & $0.519 \pm 0.090$ \\
\hline 3ci2 & Full AT & $0.295 \pm 0.008$ & $0.185 \pm 0.012$ & $0.174 \pm 0.013$ & $0.176 \pm 0.019$ & $0.208 \pm 0.051$ \\
& Full CG & $0.323 \pm 0.009$ & $0.482 \pm 0.020$ & $0.314 \pm 0.012$ & & $0.373 \pm 0.077$ \\
& Layer Sch. 2 & $0.314 \pm 0.008$ & $0.304 \pm 0.007$ & $0.300 \pm 0.007$ & & $0.306 \pm 0.006$ \\
\hline 3eye & Full AT & $0.274 \pm 0.018$ & $0.267 \pm 0.012$ & $0.312 \pm 0.023$ & $0.346 \pm 0.013$ & $0.300 \pm 0.032$ \\
& Full CG & $0.345 \pm 0.022$ & $0.366 \pm 0.018$ & $0.326 \pm 0.015$ & & $0.349 \pm 0.013$ \\
& Layer Sch. 2 & $0.243 \pm 0.010$ & $0.183 \pm 0.012$ & $0.220 \pm 0.008$ & & $0.215 \pm 0.025$ \\
\hline
\end{tabular}


Table S2: Average radius of gyration (RGYR) over the last 5 ns of simulation for the 3-4 replicas and averaged over the replicas. For the individual replicas, the uncertainty estimate is the standard deviation over the frames in the $5 \mathrm{~ns}$. For the average over replicas, the uncertainty estimate is the standard deviation over the averages of the individual replicas.

\begin{tabular}{|c|c|c|c|c|c|c|}
\hline Protein & Method & Rep. 1 & Rep. 2 & Rep. 3 & Rep. 4 & Average \\
\hline \multirow[t]{3}{*}{ 1aki } & Full AT & $1.348 \pm 0.011$ & $1.354 \pm 0.008$ & $1.350 \pm 0.009$ & $1.365 \pm 0.008$ & $1.354 \pm 0.006$ \\
\hline & Full CG & $1.384 \pm 0.008$ & $1.441 \pm 0.006$ & $1.447 \pm 0.007$ & & $1.424 \pm 0.029$ \\
\hline & Layer Sch. 2 & $1.347 \pm 0.007$ & $1.347 \pm 0.007$ & $1.355 \pm 0.006$ & & $1.349 \pm 0.004$ \\
\hline \multirow[t]{3}{*}{$1 \mathrm{e} 8 \mathrm{I}$} & Full AT & $1.367 \pm 0.010$ & $1.401 \pm 0.007$ & $1.324 \pm 0.006$ & $1.375 \pm 0.008$ & $1.367 \pm 0.028$ \\
\hline & Full CG & $1.361 \pm 0.009$ & $1.420 \pm 0.006$ & $1.448 \pm 0.008$ & & $1.410 \pm 0.036$ \\
\hline & Layer Sch. 2 & $1.341 \pm 0.007$ & $1.359 \pm 0.006$ & $1.347 \pm 0.007$ & & $1.349 \pm 0.008$ \\
\hline \multirow[t]{3}{*}{$1 \mathrm{mvg}$} & Full AT & $1.366 \pm 0.006$ & $1.345 \pm 0.007$ & $1.421 \pm 0.010$ & $1.370 \pm 0.008$ & $1.375 \pm 0.028$ \\
\hline & Full CG & $1.360 \pm 0.007$ & $1.417 \pm 0.006$ & $1.425 \pm 0.007$ & & $1.401 \pm 0.029$ \\
\hline & Layer Sch. 2 & $1.356 \pm 0.007$ & $1.350 \pm 0.010$ & $1.342 \pm 0.006$ & & $1.349 \pm 0.006$ \\
\hline \multirow[t]{3}{*}{$1 \mathrm{qk} 8$} & Full AT & $1.389 \pm 0.006$ & $1.411 \pm 0.011$ & $1.396 \pm 0.007$ & $1.381 \pm 0.006$ & $1.394 \pm 0.011$ \\
\hline & Full CG & $1.386 \pm 0.007$ & $1.452 \pm 0.005$ & $1.434 \pm 0.005$ & & $1.424 \pm 0.028$ \\
\hline & Layer Sch. 2 & $1.407 \pm 0.006$ & $1.409 \pm 0.006$ & $1.395 \pm 0.006$ & & $1.404 \pm 0.006$ \\
\hline \multirow[t]{3}{*}{ 1qqv } & Full AT & $1.121 \pm 0.010$ & $1.113 \pm 0.015$ & $1.068 \pm 0.009$ & $1.081 \pm 0.010$ & $1.096 \pm 0.022$ \\
\hline & Full CG & $1.182 \pm 0.015$ & $1.386 \pm 0.017$ & $1.342 \pm 0.017$ & & $1.303 \pm 0.088$ \\
\hline & Layer Sch. 2 & $1.165 \pm 0.012$ & $1.152 \pm 0.016$ & $1.190 \pm 0.016$ & & $1.169 \pm 0.016$ \\
\hline \multirow[t]{3}{*}{$1 \mathrm{tvq}$} & Full AT & $1.357 \pm 0.007$ & $1.370 \pm 0.007$ & $1.362 \pm 0.009$ & $1.316 \pm 0.005$ & $1.351 \pm 0.021$ \\
\hline & Full CG & $1.332 \pm 0.006$ & $1.391 \pm 0.005$ & $1.382 \pm 0.005$ & & $1.368 \pm 0.026$ \\
\hline & Layer Sch. 2 & $1.404 \pm 0.009$ & $1.398 \pm 0.007$ & $1.389 \pm 0.010$ & & $1.397 \pm 0.006$ \\
\hline \multirow[t]{3}{*}{1 ucs } & Full AT & $1.007 \pm 0.008$ & $1.023 \pm 0.007$ & $1.018 \pm 0.007$ & $1.033 \pm 0.008$ & $1.020 \pm 0.009$ \\
\hline & Full CG & $0.999 \pm 0.006$ & $1.094 \pm 0.010$ & $1.110 \pm 0.007$ & & $1.067 \pm 0.049$ \\
\hline & Layer Sch. 2 & $0.995 \pm 0.005$ & $0.997 \pm 0.008$ & $1.006 \pm 0.007$ & & $0.999 \pm 0.005$ \\
\hline \multirow[t]{3}{*}{$1 \mathrm{uxz}$} & Full AT & $1.351 \pm 0.009$ & $1.383 \pm 0.009$ & $1.356 \pm 0.007$ & $1.359 \pm 0.006$ & $1.362 \pm 0.012$ \\
\hline & Full CG & $1.352 \pm 0.008$ & $1.408 \pm 0.009$ & $1.368 \pm 0.006$ & & $1.376 \pm 0.024$ \\
\hline & Layer Sch. 2 & $1.335 \pm 0.005$ & $1.336 \pm 0.006$ & $1.342 \pm 0.006$ & & $1.338 \pm 0.003$ \\
\hline \multirow[t]{3}{*}{$1 \mathrm{zvg}$} & Full AT & $1.036 \pm 0.006$ & $1.034 \pm 0.005$ & $1.048 \pm 0.006$ & $1.063 \pm 0.008$ & $1.045 \pm 0.012$ \\
\hline & Full CG & $1.036 \pm 0.007$ & $1.154 \pm 0.011$ & $1.176 \pm 0.009$ & & $1.122 \pm 0.061$ \\
\hline & Layer Sch. 2 & $1.063 \pm 0.007$ & $1.041 \pm 0.008$ & $1.068 \pm 0.008$ & & $1.057 \pm 0.011$ \\
\hline \multirow[t]{3}{*}{$2 c w r$} & Full AT & $1.274 \pm 0.007$ & $1.304 \pm 0.010$ & $1.269 \pm 0.007$ & $1.305 \pm 0.010$ & $1.288 \pm 0.017$ \\
\hline & Full CG & $1.286 \pm 0.008$ & $1.305 \pm 0.007$ & $1.314 \pm 0.007$ & & $1.301 \pm 0.012$ \\
\hline & Layer Sch. 2 & $1.286 \pm 0.007$ & $1.287 \pm 0.009$ & $1.279 \pm 0.007$ & & $1.284 \pm 0.004$ \\
\hline \multirow[t]{3}{*}{$2 c z n$} & Full AT & $1.286 \pm 0.007$ & $1.300 \pm 0.011$ & $1.288 \pm 0.007$ & $1.298 \pm 0.007$ & $1.293 \pm 0.006$ \\
\hline & Full CG & $1.260 \pm 0.006$ & $1.304 \pm 0.007$ & $1.305 \pm 0.006$ & & $1.290 \pm 0.021$ \\
\hline & Layer Sch. 2 & $1.284 \pm 0.006$ & $1.286 \pm 0.006$ & $1.279 \pm 0.007$ & & $1.283 \pm 0.003$ \\
\hline \multirow[t]{3}{*}{$2 \mathrm{gkt}$} & Full AT & $1.018 \pm 0.009$ & $1.045 \pm 0.012$ & $1.008 \pm 0.013$ & $0.971 \pm 0.013$ & $1.010 \pm 0.027$ \\
\hline & Full CG & $0.996 \pm 0.010$ & $1.060 \pm 0.008$ & $1.072 \pm 0.009$ & & $1.043 \pm 0.034$ \\
\hline & Layer Sch. 2 & $0.981 \pm 0.007$ & $1.062 \pm 0.011$ & $1.040 \pm 0.010$ & & $1.028 \pm 0.034$ \\
\hline \multirow[t]{3}{*}{$2 \mathrm{j} 8 \mathrm{~b}$} & Full AT & $1.123 \pm 0.014$ & $1.162 \pm 0.012$ & $1.135 \pm 0.010$ & $1.104 \pm 0.005$ & $1.131 \pm 0.021$ \\
\hline & Full CG & $1.128 \pm 0.007$ & $1.200 \pm 0.010$ & $1.265 \pm 0.010$ & & $1.198 \pm 0.056$ \\
\hline & Layer Sch. 2 & $1.139 \pm 0.010$ & $1.134 \pm 0.006$ & $1.116 \pm 0.008$ & & $1.130 \pm 0.010$ \\
\hline \multirow[t]{3}{*}{ 2nls } & Full AT & $0.815 \pm 0.008$ & $0.848 \pm 0.007$ & $0.827 \pm 0.008$ & $0.836 \pm 0.007$ & $0.832 \pm 0.012$ \\
\hline & Full CG & $0.819 \pm 0.006$ & $0.984 \pm 0.010$ & $0.943 \pm 0.010$ & & $0.915 \pm 0.070$ \\
\hline & Layer Sch. 2 & $0.811 \pm 0.008$ & $0.817 \pm 0.009$ & $0.829 \pm 0.007$ & & $0.819 \pm 0.007$ \\
\hline \multirow[t]{3}{*}{ 2pnd } & Full AT & $1.412 \pm 0.007$ & $1.421 \pm 0.008$ & $1.412 \pm 0.007$ & $1.410 \pm 0.007$ & $1.414 \pm 0.004$ \\
\hline & Full CG & $1.390 \pm 0.005$ & $1.478 \pm 0.007$ & $1.470 \pm 0.006$ & & $1.446 \pm 0.039$ \\
\hline & Layer Sch. 2 & $1.419 \pm 0.006$ & $1.421 \pm 0.007$ & $1.412 \pm 0.006$ & & $1.417 \pm 0.004$ \\
\hline \multirow[t]{3}{*}{$2 p n e$} & Full AT & $1.327 \pm 0.006$ & $1.328 \pm 0.007$ & $1.322 \pm 0.007$ & $1.329 \pm 0.007$ & $1.326 \pm 0.003$ \\
\hline & Full CG & $1.311 \pm 0.008$ & $1.366 \pm 0.008$ & $1.356 \pm 0.009$ & & $1.344 \pm 0.024$ \\
\hline & Layer Sch. 2 & $1.333 \pm 0.007$ & $1.336 \pm 0.008$ & $1.318 \pm 0.007$ & & $1.329 \pm 0.008$ \\
\hline 2pth & Full AT & $1.544 \pm 0.005$ & $1.537 \pm 0.006$ & $1.529 \pm 0.006$ & $1.542 \pm 0.005$ & $1.538 \pm 0.006$ \\
\hline
\end{tabular}




\begin{tabular}{|c|c|c|c|c|c|c|}
\hline & Full CG & $1.579 \pm 0.008$ & $1.675 \pm 0.007$ & $1.805 \pm 0.014$ & & $1.687 \pm 0.093$ \\
\hline & Layer Sch. 2 & $1.538 \pm 0.006$ & $1.566 \pm 0.008$ & $1.543 \pm 0.009$ & & $1.549 \pm 0.012$ \\
\hline \multirow[t]{3}{*}{2 wlw } & Full AT & $1.402 \pm 0.005$ & $1.427 \pm 0.006$ & $1.406 \pm 0.005$ & \multirow[t]{3}{*}{$1.413 \pm 0.005$} & $1.412 \pm 0.010$ \\
\hline & Full CG & $1.399 \pm 0.004$ & $1.472 \pm 0.005$ & $1.480 \pm 0.007$ & & $1.450 \pm 0.036$ \\
\hline & Layer Sch. 2 & $1.417 \pm 0.005$ & $1.412 \pm 0.004$ & $1.418 \pm 0.004$ & & $1.415 \pm 0.003$ \\
\hline \multirow[t]{3}{*}{$2 y x f$} & Full AT & $1.376 \pm 0.010$ & $1.376 \pm 0.009$ & $1.411 \pm 0.007$ & \multirow[t]{3}{*}{$1.377 \pm 0.012$} & $1.385 \pm 0.015$ \\
\hline & Full CG & $1.385 \pm 0.008$ & $1.420 \pm 0.007$ & $1.405 \pm 0.007$ & & $1.403 \pm 0.015$ \\
\hline & Layer Sch. 2 & $1.347 \pm 0.009$ & $1.376 \pm 0.009$ & $1.373 \pm 0.009$ & & $1.365 \pm 0.013$ \\
\hline \multirow[t]{3}{*}{ 3ca7 } & Full AT & $1.109 \pm 0.016$ & $1.081 \pm 0.012$ & $1.052 \pm 0.013$ & \multirow[t]{3}{*}{$1.025 \pm 0.029$} & $1.067 \pm 0.031$ \\
\hline & Full CG & $1.068 \pm 0.010$ & $1.149 \pm 0.013$ & $1.173 \pm 0.010$ & & $1.130 \pm 0.045$ \\
\hline & Layer Sch. 2 & $1.005 \pm 0.013$ & $0.954 \pm 0.008$ & $1.001 \pm 0.012$ & & $0.987 \pm 0.023$ \\
\hline \multirow[t]{3}{*}{$3 c i 2$} & Full AT & $1.054 \pm 0.007$ & $1.049 \pm 0.007$ & $1.059 \pm 0.008$ & \multirow[t]{3}{*}{$1.044 \pm 0.007$} & $1.052 \pm 0.005$ \\
\hline & Full CG & $1.048 \pm 0.007$ & $1.211 \pm 0.013$ & $1.146 \pm 0.009$ & & $1.135 \pm 0.067$ \\
\hline & Layer Sch. 2 & $1.027 \pm 0.007$ & $1.027 \pm 0.006$ & $1.032 \pm 0.007$ & & $1.029 \pm 0.002$ \\
\hline \multirow[t]{3}{*}{ 3eye } & Full AT & $1.437 \pm 0.010$ & $1.459 \pm 0.006$ & $1.441 \pm 0.006$ & \multirow[t]{3}{*}{$1.454 \pm 0.007$} & $1.448 \pm 0.009$ \\
\hline & Full CG & $1.472 \pm 0.007$ & $1.535 \pm 0.006$ & $1.551 \pm 0.009$ & & $1.519 \pm 0.034$ \\
\hline & Layer Sch. 2 & $1.447 \pm 0.007$ & $1.443 \pm 0.006$ & $1.446 \pm 0.006$ & & $1.445 \pm 0.001$ \\
\hline
\end{tabular}


Table S3: Average total number of backbone-backbone hydrogen bonds over the last 5 ns of simulation for the 3-4 replicas and averaged over the replicas. For the individual replicas, the uncertainty estimate is the standard deviation over the frames in the $5 \mathrm{~ns}$. For the average over replicas, the uncertainty estimate is the standard deviation over the averages of the individual replicas.

\begin{tabular}{|c|c|c|c|c|c|c|}
\hline Protein & Method & Rep. 1 & Rep. 2 & Rep. 3 & Rep. 4 & Average \\
\hline \multirow[t]{3}{*}{ 1aki } & Full AT & 90 & 87 & 83 & 87 & $86.8 \pm 2.5$ \\
\hline & Full CG & 106 & 96 & 92 & - & $98.0 \pm 5.9$ \\
\hline & Layer Sch. 2 & 93 & 96 & 86 & - & $91.7 \pm 4.2$ \\
\hline \multirow[t]{3}{*}{$1 \mathrm{e} 8 \mathrm{I}$} & Full AT & 80 & 81 & 82 & 81 & $81.0 \pm 0.7$ \\
\hline & Full CG & 93 & 97 & 100 & - & $96.7 \pm 2.9$ \\
\hline & Layer Sch. 2 & 93 & 99 & 89 & - & $93.7 \pm 4.1$ \\
\hline \multirow[t]{3}{*}{$1 \mathrm{mvg}$} & Full AT & 86 & 85 & 79 & 90 & $85.0 \pm 3.9$ \\
\hline & Full CG & 102 & 101 & 87 & - & $96.7 \pm 6.8$ \\
\hline & Layer Sch. 2 & 88 & 97 & 90 & - & $91.7 \pm 3.9$ \\
\hline \multirow[t]{3}{*}{$1 q k 8$} & Full AT & 90 & 93 & 83 & 93 & $89.8 \pm 4.1$ \\
\hline & Full CG & 103 & 107 & 107 & - & $105.7 \pm 1.9$ \\
\hline & Layer Sch. 2 & 94 & 92 & 91 & - & $92.3 \pm 1.2$ \\
\hline \multirow[t]{3}{*}{ 1qqv } & Full AT & 38 & 37 & 37 & 32 & $36.0 \pm 2.3$ \\
\hline & Full CG & 46 & 42 & 50 & - & $46.0 \pm 3.3$ \\
\hline & Layer Sch. 2 & 43 & 44 & 39 & - & $42.0 \pm 2.2$ \\
\hline \multirow[t]{3}{*}{1 tvq } & Full AT & 82 & 84 & 82 & 88 & $84.0 \pm 2.4$ \\
\hline & Full CG & 106 & 92 & 84 & - & $94.0 \pm 9.1$ \\
\hline & Layer Sch. 2 & 95 & 96 & 84 & - & $91.7 \pm 5.4$ \\
\hline \multirow[t]{3}{*}{ 1ucs } & Full AT & 37 & 37 & 35 & 37 & $36.5 \pm 0.9$ \\
\hline & Full CG & 39 & 37 & 42 & - & $39.3 \pm 2.1$ \\
\hline & Layer Sch. 2 & 38 & 45 & 41 & - & $41.3 \pm 2.9$ \\
\hline \multirow[t]{3}{*}{$1 \mathrm{uxz}$} & Full AT & 85 & 82 & 79 & 74 & $80.0 \pm 4.1$ \\
\hline & Full CG & 84 & 91 & 90 & - & $88.3 \pm 3.1$ \\
\hline & Layer Sch. 2 & 82 & 88 & 83 & - & $84.3 \pm 2.6$ \\
\hline \multirow[t]{3}{*}{$1 z v g$} & Full AT & 33 & 32 & 33 & 34 & $33.0 \pm 0.7$ \\
\hline & Full CG & 46 & 41 & 45 & - & $44.0 \pm 2.2$ \\
\hline & Layer Sch. 2 & 42 & 38 & 50 & - & $43.3 \pm 5.0$ \\
\hline \multirow[t]{3}{*}{$2 c w r$} & Full AT & 58 & 55 & 59 & 63 & $58.8 \pm 2.9$ \\
\hline & Full CG & 55 & 67 & 58 & - & $60.0 \pm 5.1$ \\
\hline & Layer Sch. 2 & 61 & 66 & 65 & - & $64.0 \pm 2.2$ \\
\hline \multirow[t]{3}{*}{$2 c z n$} & Full AT & 61 & 66 & 60 & 65 & $63.0 \pm 2.5$ \\
\hline & Full CG & 63 & 65 & 64 & - & $64.0 \pm 0.8$ \\
\hline & Layer Sch. 2 & 69 & 75 & 67 & - & $70.3 \pm 3.4$ \\
\hline \multirow[t]{3}{*}{$2 \mathrm{gkt}$} & Full AT & 25 & 21 & 17 & 30 & $23.2 \pm 4.8$ \\
\hline & Full CG & 27 & 23 & 28 & - & $26.0 \pm 2.2$ \\
\hline & Layer Sch. 2 & 30 & 30 & 30 & - & $30.0 \pm 0.0$ \\
\hline \multirow[t]{3}{*}{$2 \mathrm{j} 8 \mathrm{~b}$} & Full AT & 45 & 38 & 39 & 36 & $39.5 \pm 3.4$ \\
\hline & Full CG & 51 & 51 & 54 & - & $52.0 \pm 1.4$ \\
\hline & Layer Sch. 2 & 51 & 37 & 48 & - & $45.3 \pm 6.0$ \\
\hline \multirow[t]{3}{*}{$2 \mathrm{nls}$} & Full AT & 22 & 21 & 22 & 21 & $21.5 \pm 0.5$ \\
\hline & Full CG & 23 & 18 & 20 & - & $20.3 \pm 2.1$ \\
\hline & Layer Sch. 2 & 24 & 22 & 24 & - & $23.3 \pm 0.9$ \\
\hline \multirow[t]{3}{*}{ 2pnd } & Full AT & 73 & 73 & 73 & 76 & $73.8 \pm 1.3$ \\
\hline & Full CG & 73 & 76 & 78 & - & $75.7 \pm 2.1$ \\
\hline & Layer Sch. 2 & 71 & 73 & 69 & - & $71.0 \pm 1.6$ \\
\hline \multirow[t]{2}{*}{ 2pne } & Full AT & 42 & 41 & 41 & 42 & $41.5 \pm 0.5$ \\
\hline & Full CG & 47 & 46 & 45 & - & $46.0 \pm 0.8$ \\
\hline
\end{tabular}




\begin{tabular}{lllllll} 
& Layer Sch. 2 & 41 & 39 & 36 & - & $38.7 \pm 2.1$ \\
\hline 2pth & Full AT & 133 & 135 & 128 & 137 & $133.2 \pm 3.3$ \\
& Full CG & 154 & 143 & 145 & - & $147.3 \pm 4.8$ \\
& Layer Sch. 2 & 140 & 151 & 151 & - & $147.3 \pm 5.2$ \\
\hline \multirow{2}{*}{ wlw } & Full AT & 111 & 104 & 108 & 106 & $107.2 \pm 2.6$ \\
& Full CG & 127 & 117 & 122 & - & $122.0 \pm 4.1$ \\
& Layer Sch. 2 & 116 & 114 & 116 & - & $115.3 \pm 0.9$ \\
\hline 2yxf & Full AT & 51 & 57 & 47 & 53 & $52.0 \pm 3.6$ \\
& Full CG & 59 & 59 & 56 & - & $58.0 \pm 1.4$ \\
& Layer Sch. 2 & 60 & 59 & 64 & - & $61.0 \pm 2.2$ \\
\hline \multirow{3}{3}{ ca7 } & Full AT & 27 & 25 & 25 & 27 & $26.0 \pm 1.0$ \\
& Full CG & 29 & 32 & 33 & - & $31.3 \pm 1.7$ \\
& Layer Sch. 2 & 29 & 26 & 26 & - & $27.0 \pm 1.4$ \\
\hline \multirow{3}{3}{ ci2 } & Full AT & 40 & 36 & 38 & 38 & $38.0 \pm 1.4$ \\
& Full CG & 43 & 36 & 45 & - & $41.3 \pm 3.9$ \\
& Layer Sch. 2 & 42 & 37 & 39 & - & $39.3 \pm 2.1$ \\
\hline 3eye & Full AT & 112 & 101 & 107 & 108 & $107.0 \pm 3.9$ \\
& Full CG & 114 & 111 & 113 & - & $112.7 \pm 1.2$ \\
& Layer Sch. 2 & 103 & 109 & 105 & - & $105.7 \pm 2.5$ \\
\hline
\end{tabular}


Table S4: Average total number of backbone-side chain hydrogen bonds over the last 5 ns of simulation for the 3-4 replicas and averaged over the replicas. For the individual replicas, the uncertainty estimate is the standard deviation over the frames in the $5 \mathrm{~ns}$. For the average over replicas, the uncertainty estimate is the standard deviation over the averages of the individual replicas.

\begin{tabular}{|c|c|c|c|c|c|c|}
\hline Protein & Method & Rep. 1 & Rep. 2 & Rep. 3 & Rep. 4 & Average \\
\hline \multirow[t]{3}{*}{ 1aki } & Full AT & 55 & 59 & 54 & 61 & $57.2 \pm 2.9$ \\
\hline & Full CG & 89 & 69 & 72 & - & $76.7 \pm 8.8$ \\
\hline & Layer Sch. 2 & 57 & 60 & 47 & - & $54.7 \pm 5.6$ \\
\hline \multirow[t]{3}{*}{$1 \mathrm{e} 8 \mathrm{I}$} & Full AT & 52 & 68 & 63 & 47 & $57.5 \pm 8.4$ \\
\hline & Full CG & 75 & 63 & 67 & - & $68.3 \pm 5.0$ \\
\hline & Layer Sch. 2 & 54 & 51 & 48 & - & $51.0 \pm 2.4$ \\
\hline \multirow[t]{3}{*}{$1 \mathrm{mvg}$} & Full AT & 48 & 45 & 52 & 48 & $48.2 \pm 2.5$ \\
\hline & Full CG & 61 & 68 & 69 & - & $66.0 \pm 3.6$ \\
\hline & Layer Sch. 2 & 48 & 43 & 37 & - & $42.7 \pm 4.5$ \\
\hline \multirow[t]{3}{*}{$19 k 8$} & Full AT & 65 & 74 & 71 & 63 & $68.2 \pm 4.4$ \\
\hline & Full CG & 68 & 65 & 78 & - & $70.3 \pm 5.6$ \\
\hline & Layer Sch. 2 & 58 & 59 & 72 & - & $63.0 \pm 6.4$ \\
\hline \multirow[t]{3}{*}{ 1qqv } & Full AT & 41 & 32 & 45 & 37 & $38.8 \pm 4.8$ \\
\hline & Full CG & 30 & 41 & 36 & - & $35.7 \pm 4.5$ \\
\hline & Layer Sch. 2 & 36 & 26 & 22 & - & $28.0 \pm 5.9$ \\
\hline \multirow[t]{3}{*}{1 tvq } & Full AT & 42 & 37 & 39 & 40 & $39.5 \pm 1.8$ \\
\hline & Full CG & 59 & 87 & 92 & - & $79.3 \pm 14.5$ \\
\hline & Layer Sch. 2 & 35 & 44 & 46 & - & $41.7 \pm 4.8$ \\
\hline \multirow[t]{3}{*}{ 1ucs } & Full AT & 17 & 20 & 14 & 21 & $18.0 \pm 2.7$ \\
\hline & Full CG & 25 & 32 & 33 & - & $30.0 \pm 3.6$ \\
\hline & Layer Sch. 2 & 20 & 22 & 21 & - & $21.0 \pm 0.8$ \\
\hline \multirow[t]{3}{*}{$1 \mathrm{uxz}$} & Full AT & 50 & 52 & 53 & 52 & $51.8 \pm 1.1$ \\
\hline & Full CG & 61 & 63 & 62 & - & $62.0 \pm 0.8$ \\
\hline & Layer Sch. 2 & 65 & 65 & 65 & - & $65.0 \pm 0.0$ \\
\hline \multirow[t]{3}{*}{$1 z v g$} & Full AT & 25 & 29 & 24 & 27 & $26.2 \pm 1.9$ \\
\hline & Full CG & 23 & 34 & 30 & - & $29.0 \pm 4.5$ \\
\hline & Layer Sch. 2 & 25 & 38 & 29 & - & $30.7 \pm 5.4$ \\
\hline \multirow[t]{3}{*}{$2 c w r$} & Full AT & 28 & 28 & 25 & 23 & $26.0 \pm 2.1$ \\
\hline & Full CG & 70 & 42 & 51 & - & $54.3 \pm 11.7$ \\
\hline & Layer Sch. 2 & 33 & 32 & 36 & - & $33.7 \pm 1.7$ \\
\hline \multirow[t]{3}{*}{$2 c z n$} & Full AT & 29 & 20 & 28 & 28 & $26.2 \pm 3.6$ \\
\hline & Full CG & 55 & 44 & 58 & - & $52.3 \pm 6.0$ \\
\hline & Layer Sch. 2 & 32 & 36 & 31 & - & $33.0 \pm 2.2$ \\
\hline \multirow[t]{3}{*}{$2 \mathrm{gkt}$} & Full AT & 28 & 14 & 27 & 22 & $22.8 \pm 5.5$ \\
\hline & Full CG & 27 & 30 & 27 & - & $28.0 \pm 1.4$ \\
\hline & Layer Sch. 2 & 25 & 21 & 17 & - & $21.0 \pm 3.3$ \\
\hline \multirow[t]{3}{*}{$2 \mathrm{j} 8 \mathrm{~b}$} & Full AT & 33 & 41 & 36 & 43 & $38.2 \pm 4.0$ \\
\hline & Full CG & 48 & 51 & 51 & - & $50.0 \pm 1.4$ \\
\hline & Layer Sch. 2 & 43 & 38 & 43 & - & $41.3 \pm 2.4$ \\
\hline \multirow[t]{3}{*}{$2 \mathrm{nls}$} & Full AT & 13 & 11 & 11 & 7 & $10.5 \pm 2.2$ \\
\hline & Full CG & 25 & 31 & 35 & - & $30.3 \pm 4.1$ \\
\hline & Layer Sch. 2 & 14 & 18 & 18 & - & $16.7 \pm 1.9$ \\
\hline \multirow[t]{3}{*}{ 2pnd } & Full AT & 53 & 49 & 40 & 52 & $48.5 \pm 5.1$ \\
\hline & Full CG & 72 & 70 & 76 & - & $72.7 \pm 2.5$ \\
\hline & Layer Sch. 2 & 45 & 46 & 48 & - & $46.3 \pm 1.2$ \\
\hline \multirow[t]{2}{*}{ 2pne } & Full AT & 15 & 17 & 15 & 16 & $15.8 \pm 0.8$ \\
\hline & Full CG & 17 & 17 & 14 & - & $16.0 \pm 1.4$ \\
\hline
\end{tabular}




\begin{tabular}{lllllll} 
& Layer Sch. 2 & 18 & 14 & 26 & - & $19.3 \pm 5.0$ \\
\hline 2pth & Full AT & 59 & 60 & 64 & 63 & $61.5 \pm 2.1$ \\
& Full CG & 99 & 101 & 108 & - & $102.7 \pm 3.9$ \\
& Layer Sch. 2 & 69 & 68 & 66 & - & $67.7 \pm 1.2$ \\
\hline \multirow{2}{*}{ wlw } & Full AT & 71 & 57 & 70 & 70 & $67.0 \pm 5.8$ \\
& Full CG & 67 & 82 & 84 & - & $77.7 \pm 7.6$ \\
& Layer Sch. 2 & 67 & 72 & 54 & - & $64.3 \pm 7.6$ \\
\hline 2yxf & Full AT & 29 & 41 & 49 & 44 & $40.8 \pm 7.4$ \\
& Full CG & 47 & 56 & 41 & - & $48.0 \pm 6.2$ \\
& Layer Sch. 2 & 33 & 34 & 37 & - & $34.7 \pm 1.7$ \\
\hline 3ca7 & Full AT & 18 & 19 & 20 & 15 & $18.0 \pm 1.9$ \\
& Full CG & 11 & 16 & 12 & - & $13.0 \pm 2.2$ \\
& Layer Sch. 2 & 15 & 14 & 13 & - & $14.0 \pm 0.8$ \\
\hline \multirow{3}{3}{ 3ci2 } & Full AT & 19 & 29 & 27 & 23 & $24.5 \pm 3.8$ \\
& Full CG & 34 & 37 & 42 & - & $37.7 \pm 3.3$ \\
& Layer Sch. 2 & 22 & 29 & 23 & - & $24.7 \pm 3.1$ \\
\hline \multirow{2}{*}{ 3eye } & Full AT & 77 & 79 & 86 & 75 & $79.2 \pm 4.1$ \\
& Full CG & 87 & 87 & 90 & - & $88.0 \pm 1.4$ \\
& Layer Sch. 2 & 71 & 64 & 75 & - & $70.0 \pm 4.5$ \\
\hline
\end{tabular}


Table S5: Average total number of side chain-side chain hydrogen bonds over the last 5 ns of simulation for the 3-4 replicas and averaged over the replicas. For the individual replicas, the uncertainty estimate is the standard deviation over the frames in the $5 \mathrm{~ns}$. For the average over replicas, the uncertainty estimate is the standard deviation over the averages of the individual replicas.

\begin{tabular}{|c|c|c|c|c|c|c|}
\hline Protein & Method & Rep. 1 & Rep. 2 & Rep. 3 & Rep. 4 & Average \\
\hline \multirow[t]{3}{*}{ 1aki } & Full AT & 34 & 22 & 30 & 31 & $29.2 \pm 4.4$ \\
\hline & Full CG & 36 & 38 & 31 & - & $35.0 \pm 2.9$ \\
\hline & Layer Sch. 2 & 35 & 38 & 35 & - & $36.0 \pm 1.4$ \\
\hline \multirow[t]{3}{*}{$1 \mathrm{e} 8 \mathrm{I}$} & Full AT & 26 & 35 & 37 & 25 & $30.8 \pm 5.3$ \\
\hline & Full CG & 47 & 37 & 33 & - & $39.0 \pm 5.9$ \\
\hline & Layer Sch. 2 & 23 & 38 & 33 & - & $31.3 \pm 6.2$ \\
\hline \multirow[t]{3}{*}{$1 \mathrm{mvg}$} & Full AT & 25 & 38 & 25 & 38 & $31.5 \pm 6.5$ \\
\hline & Full CG & 40 & 51 & 45 & - & $45.3 \pm 4.5$ \\
\hline & Layer Sch. 2 & 27 & 27 & 34 & - & $29.3 \pm 3.3$ \\
\hline \multirow[t]{3}{*}{ 1qk8 } & Full AT & 29 & 28 & 14 & 25 & $24.0 \pm 6.0$ \\
\hline & Full CG & 31 & 39 & 26 & - & $32.0 \pm 5.4$ \\
\hline & Layer Sch. 2 & 18 & 27 & 24 & - & $23.0 \pm 3.7$ \\
\hline \multirow[t]{3}{*}{ 1qqv } & Full AT & 16 & 11 & 7 & 12 & $11.5 \pm 3.2$ \\
\hline & Full CG & 18 & 15 & 16 & - & $16.3 \pm 1.2$ \\
\hline & Layer Sch. 2 & 17 & 12 & 11 & - & $13.3 \pm 2.6$ \\
\hline \multirow[t]{3}{*}{1 tvq } & Full AT & 36 & 29 & 40 & 32 & $34.2 \pm 4.1$ \\
\hline & Full CG & 61 & 70 & 63 & - & $64.7 \pm 3.9$ \\
\hline & Layer Sch. 2 & 28 & 38 & 27 & - & $31.0 \pm 5.0$ \\
\hline \multirow[t]{3}{*}{ 1ucs } & Full AT & 4 & 4 & 4 & 5 & $4.2 \pm 0.4$ \\
\hline & Full CG & 26 & 16 & 17 & - & $19.7 \pm 4.5$ \\
\hline & Layer Sch. 2 & 10 & 13 & 13 & - & $12.0 \pm 1.4$ \\
\hline \multirow[t]{3}{*}{$1 \mathrm{uxz}$} & Full AT & 41 & 34 & 28 & 37 & $35.0 \pm 4.7$ \\
\hline & Full CG & 39 & 40 & 56 & - & $45.0 \pm 7.8$ \\
\hline & Layer Sch. 2 & 37 & 38 & 36 & - & $37.0 \pm 0.8$ \\
\hline \multirow[t]{3}{*}{$1 z v g$} & Full AT & 13 & 10 & 13 & 14 & $12.5 \pm 1.5$ \\
\hline & Full CG & 11 & 22 & 22 & - & $18.3 \pm 5.2$ \\
\hline & Layer Sch. 2 & 12 & 13 & 15 & - & $13.3 \pm 1.2$ \\
\hline \multirow[t]{3}{*}{$2 \mathrm{cwr}$} & Full AT & 20 & 25 & 27 & 29 & $25.2 \pm 3.3$ \\
\hline & Full CG & 21 & 21 & 18 & - & $20.0 \pm 1.4$ \\
\hline & Layer Sch. 2 & 26 & 25 & 26 & - & $25.7 \pm 0.5$ \\
\hline \multirow[t]{3}{*}{$2 c z n$} & Full AT & 24 & 20 & 17 & 18 & $19.8 \pm 2.7$ \\
\hline & Full CG & 19 & 24 & 22 & - & $21.7 \pm 2.1$ \\
\hline & Layer Sch. 2 & 11 & 16 & 10 & - & $12.3 \pm 2.6$ \\
\hline \multirow[t]{3}{*}{$2 \mathrm{gkt}$} & Full AT & 17 & 16 & 8 & 13 & $13.5 \pm 3.5$ \\
\hline & Full CG & 13 & 7 & 9 & - & $9.7 \pm 2.5$ \\
\hline & Layer Sch. 2 & 11 & 9 & 9 & - & $9.7 \pm 0.9$ \\
\hline \multirow[t]{3}{*}{$2 \mathrm{j} 8 \mathrm{~b}$} & Full AT & 18 & 18 & 15 & 18 & $17.2 \pm 1.3$ \\
\hline & Full CG & 16 & 18 & 18 & - & $17.3 \pm 0.9$ \\
\hline & Layer Sch. 2 & 20 & 21 & 14 & - & $18.3 \pm 3.1$ \\
\hline \multirow[t]{3}{*}{$2 n l s$} & Full AT & 12 & 10 & 8 & 8 & $9.5 \pm 1.7$ \\
\hline & Full CG & 11 & 9 & 6 & - & $8.7 \pm 2.1$ \\
\hline & Layer Sch. 2 & 10 & 11 & 8 & - & $9.7 \pm 1.2$ \\
\hline \multirow[t]{3}{*}{ 2pnd } & Full AT & 50 & 50 & 44 & 46 & $47.5 \pm 2.6$ \\
\hline & Full CG & 44 & 53 & 55 & - & $50.7 \pm 4.8$ \\
\hline & Layer Sch. 2 & 35 & 40 & 35 & - & $36.7 \pm 2.4$ \\
\hline \multirow[t]{2}{*}{ 2pne } & Full AT & 6 & 6 & 4 & 4 & $5.0 \pm 1.0$ \\
\hline & Full CG & 3 & 7 & 7 & - & $5.7 \pm 1.9$ \\
\hline
\end{tabular}




\begin{tabular}{lllllll} 
& Layer Sch. 2 & 11 & 6 & 9 & - & $8.7 \pm 2.1$ \\
\hline 2pth & Full AT & 44 & 61 & 49 & 48 & $50.5 \pm 6.3$ \\
& Full CG & 32 & 32 & 29 & - & $31.0 \pm 1.4$ \\
& Layer Sch. 2 & 35 & 27 & 29 & - & $30.3 \pm 3.4$ \\
\hline 2wlw & Full AT & 34 & 34 & 36 & 37 & $35.2 \pm 1.3$ \\
& Full CG & 42 & 39 & 45 & - & $42.0 \pm 2.4$ \\
& Layer Sch. 2 & 44 & 37 & 35 & - & $38.7 \pm 3.9$ \\
\hline 2yxf & Full AT & 28 & 29 & 26 & 34 & $29.2 \pm 2.9$ \\
& Full CG & 30 & 36 & 27 & - & $31.0 \pm 3.7$ \\
& Layer Sch. 2 & 24 & 28 & 22 & - & $24.7 \pm 2.5$ \\
\hline \multirow{3}{*}{ ca7 } & Full AT & 4 & 3 & 8 & 5 & $5.0 \pm 1.9$ \\
& Full CG & 11 & 9 & 10 & - & $10.0 \pm 0.8$ \\
& Layer Sch. 2 & 5 & 5 & 2 & - & $4.0 \pm 1.4$ \\
\hline 3ci2 & Full AT & 12 & 8 & 11 & 10 & $10.2 \pm 1.5$ \\
& Full CG & 11 & 14 & 10 & - & $11.7 \pm 1.7$ \\
& Layer Sch. 2 & 22 & 19 & 12 & - & $17.7 \pm 4.2$ \\
\hline \multirow{2}{*}{ 3eye } & Full AT & 37 & 33 & 32 & 39 & $35.2 \pm 2.9$ \\
& Full CG & 45 & 48 & 42 & - & $45.0 \pm 2.4$ \\
& Layer Sch. 2 & 38 & 41 & 43 & - & $40.7 \pm 2.1$ \\
\hline
\end{tabular}




\section{Summary: Analyses Proteins}

\subsection{Protein 1aki}
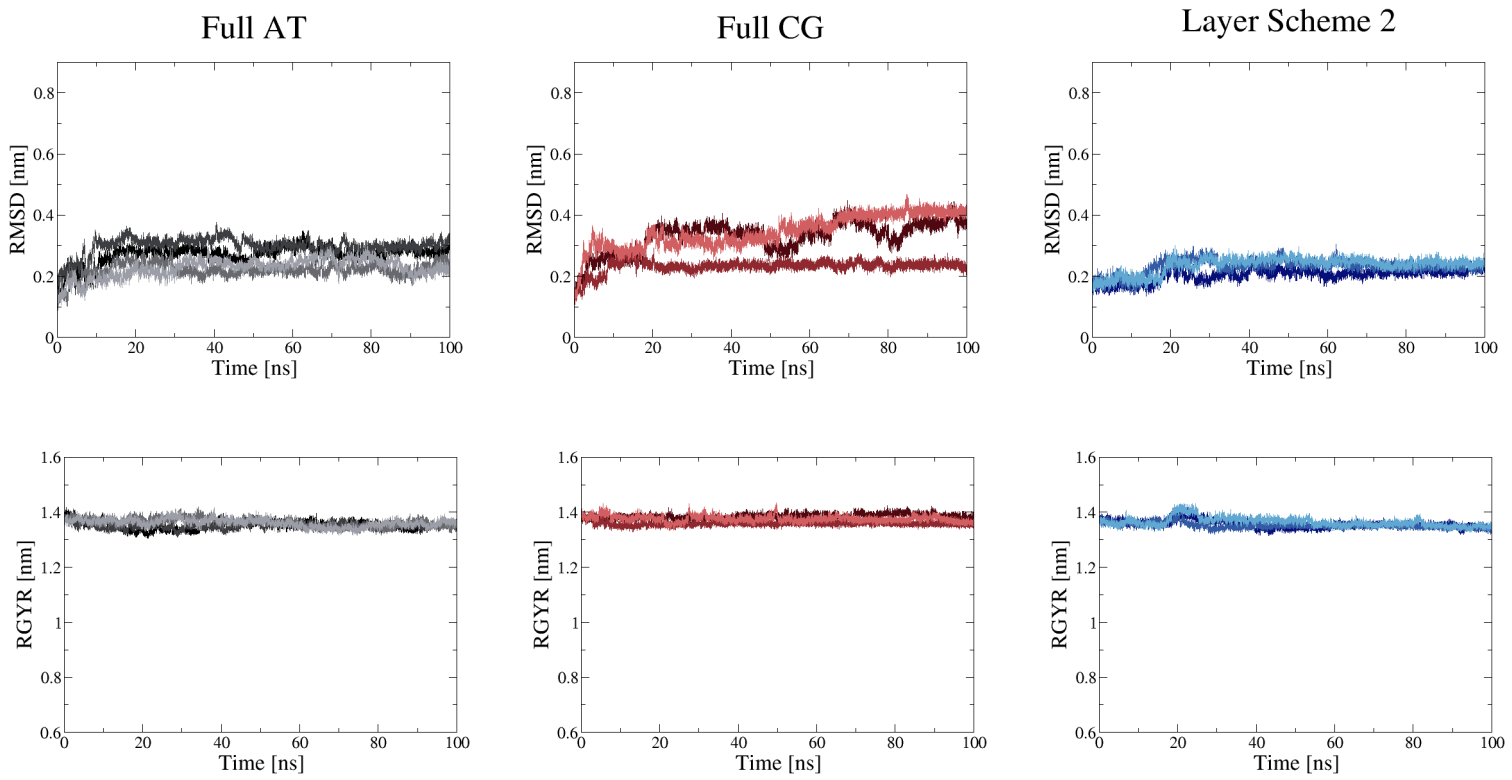

Figure S1: Time series of the backbone atom-positional root-mean-square deviation (RMSD) (top) and the radius of gyration (RGYR) (bottom) of the protein in AT water (black, left), CG water (red, middle), and AT/CG water with layer scheme 2 (blue, right). The replicas are shown in color gradients.
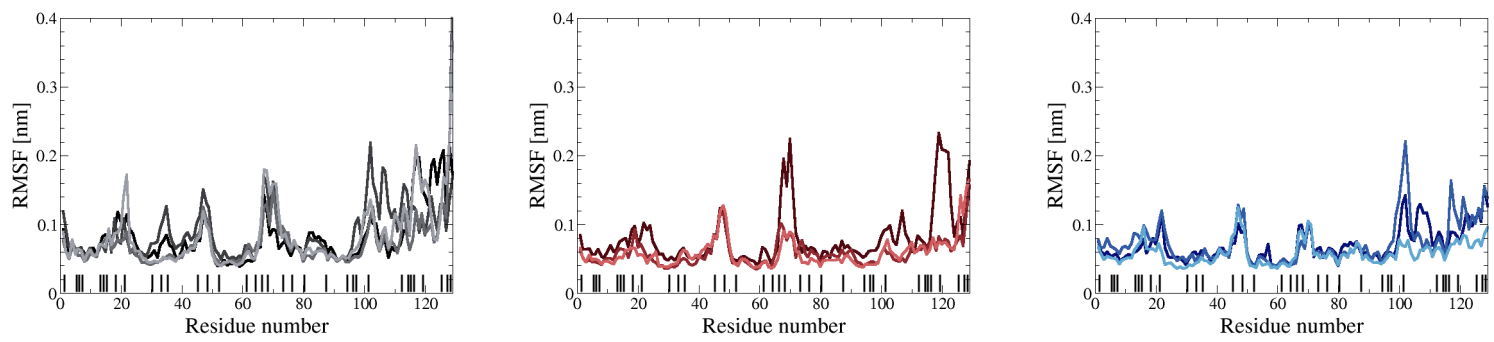

Figure S2: Root-mean-square fluctuation (RMSF) of the $C_{\alpha}$ atoms of the protein in AT water (black, left), CG water (red, middle), and AT/CG water with layer scheme 2 (blue, right). The replicas are shown in color gradients. The positions of the charged residues are indicated with a black dash on the $x$-axis. 
Full AT / Replica 1
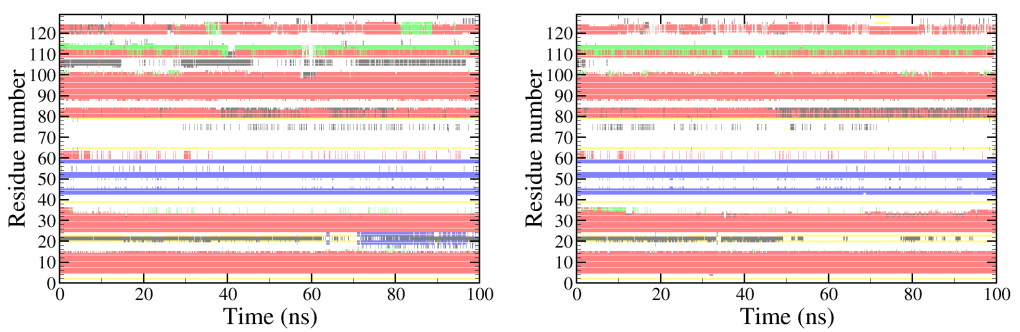

Full AT / Replica 4

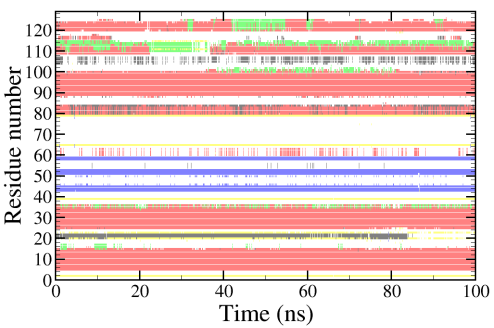

Full CG / Replica 1

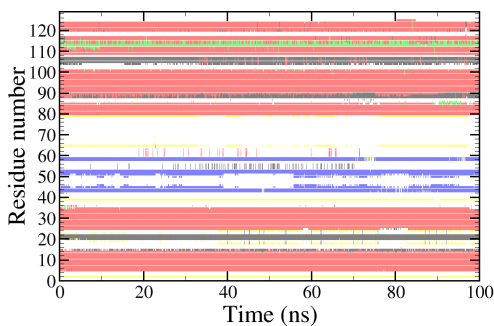

Layer Scheme 2 / Replica 1

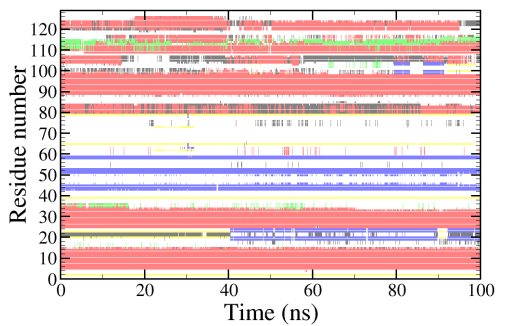

Full CG / Replica 2

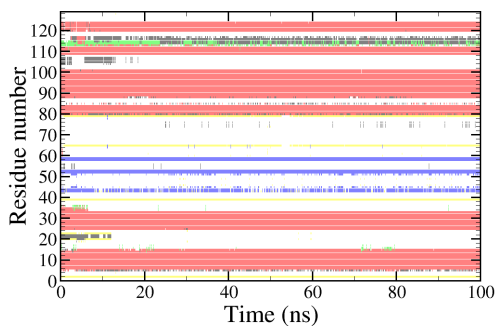

Layer Scheme 2 / Replica 2

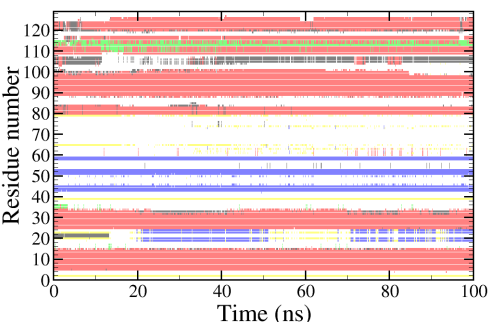

Full AT / Replica 3

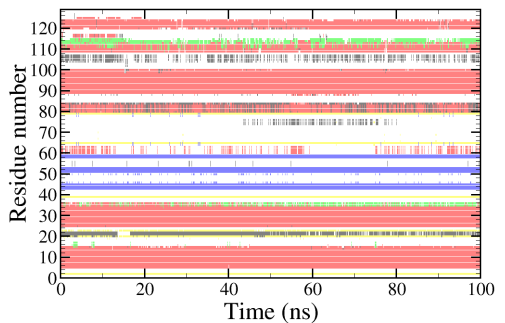

.

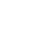




\subsection{Protein 1e8I}

Full AT
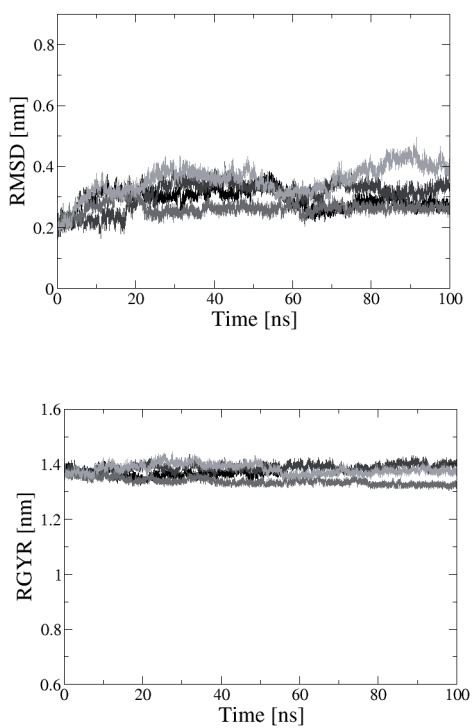

Full CG
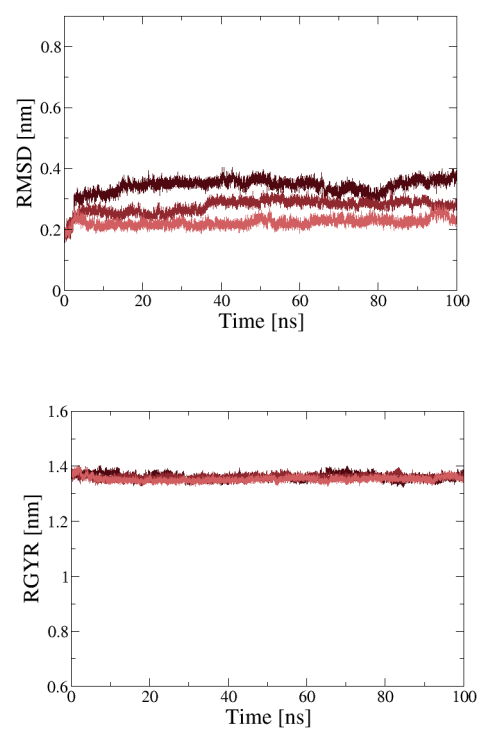

Layer Scheme 2
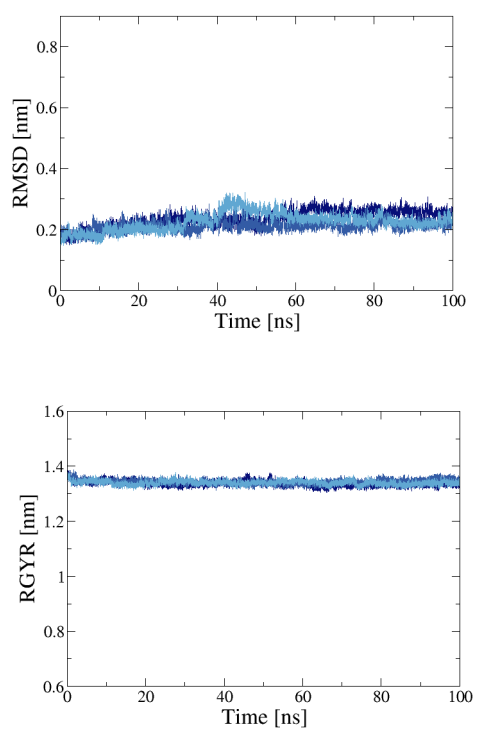

Figure S4: Time series of the backbone atom-positional root-mean-square deviation (RMSD) (top) and the radius of gyration (RGYR) (bottom) of the protein in AT water (black, left), CG water (red, middle), and AT/CG water with layer scheme 2 (blue, right). The replicas are shown in color gradients.
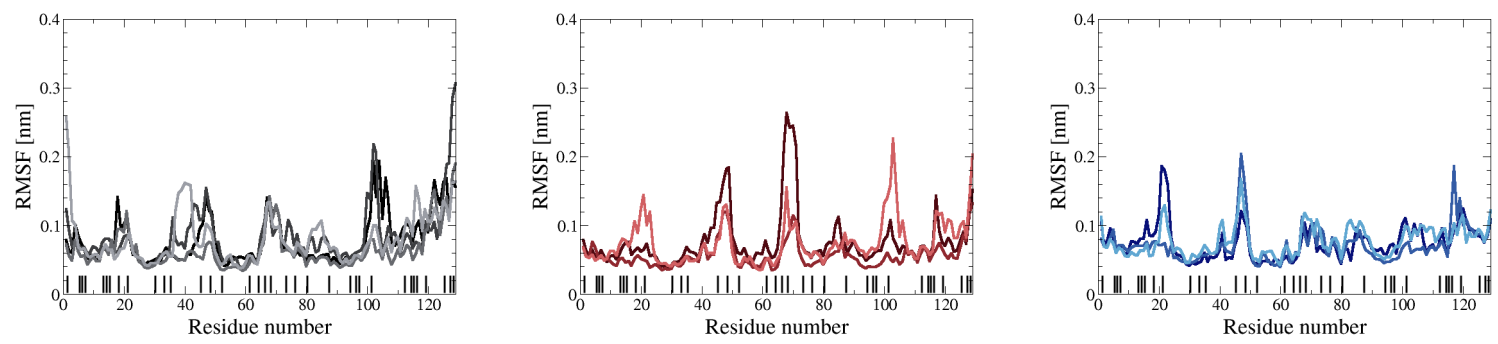

Figure S5: Root-mean-square fluctuation (RMSF) of the $C_{\alpha}$ atoms of the protein in AT water (black, left), CG water (red, middle), and AT/CG water with layer scheme 2 (blue, right). The replicas are shown in color gradients. The positions of the charged residues are indicated with a black dash on the $x$-axis. 
Full AT / Replica 1

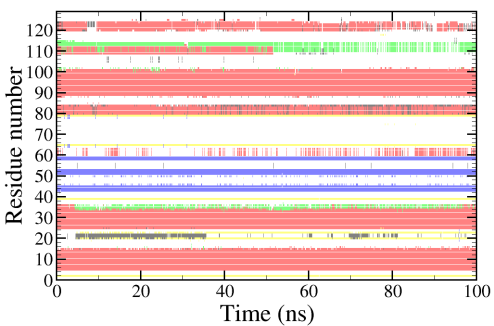

Full AT / Replica 2

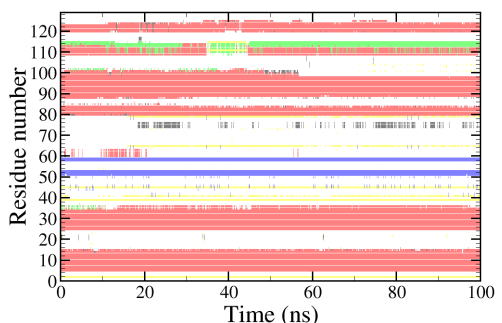

Full AT / Replica 4

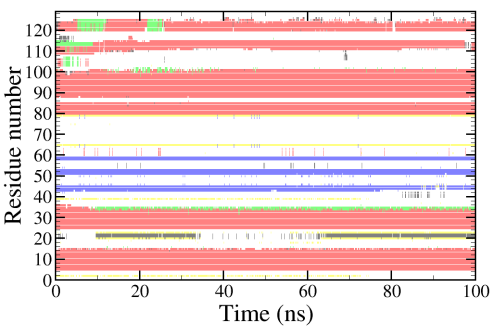

Full CG / Replica 1

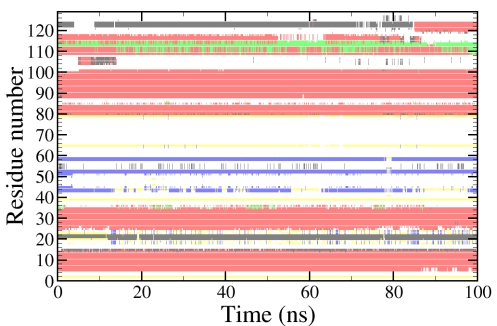

Layer Scheme 2 / Replica 1

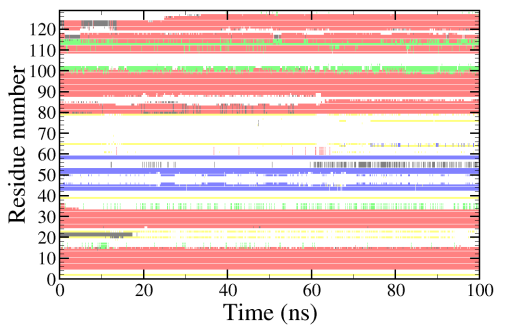

Full CG / Replica 2

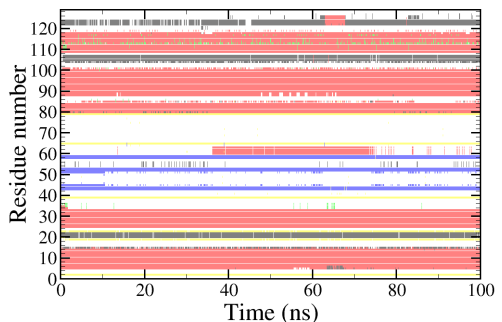

Layer Scheme 2 / Replica 2

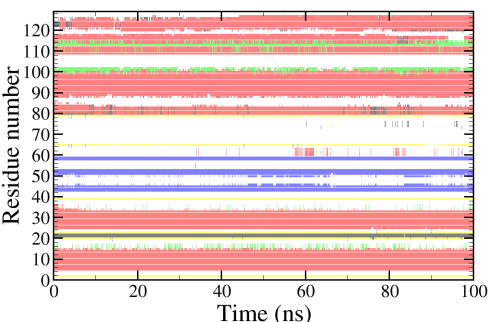

Full AT / Replica 3

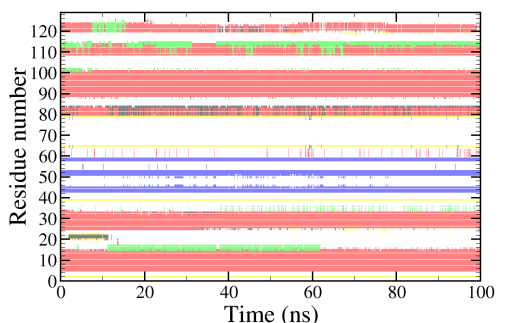

ime (ns)
Full CG / Replica 3

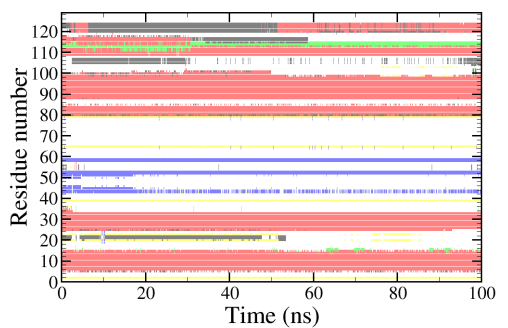

Layer Scheme 2 / Replica 3

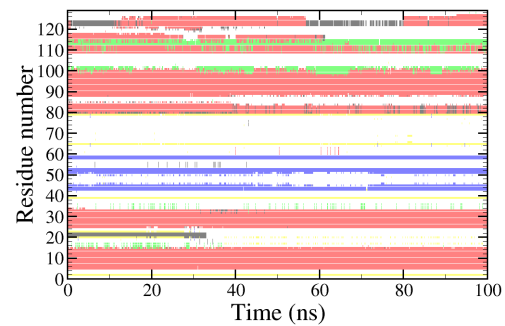

Figure S6: Time series of the secondary structure motifs of the protein in AT water (top), CG water (middle), and AT/CG water with layer scheme 2 (bottom). $\beta$-strands are shown in blue, $\beta$-bridges in yellow, $3_{10}$-helices in black, $\alpha$-helices in red, and $\pi$-helices in green. 


\subsection{Protein $1 \mathrm{mvg}$}

Full AT
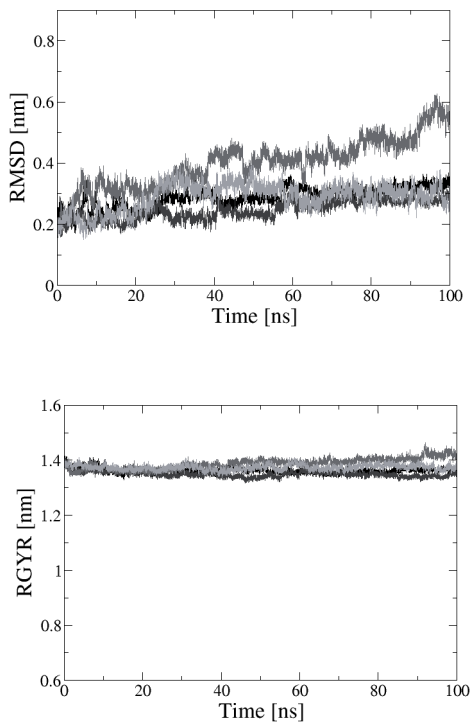

Full CG
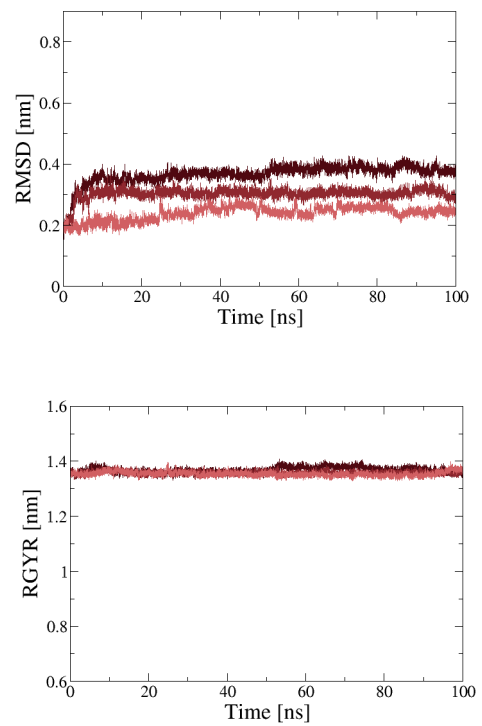

Layer Scheme 2
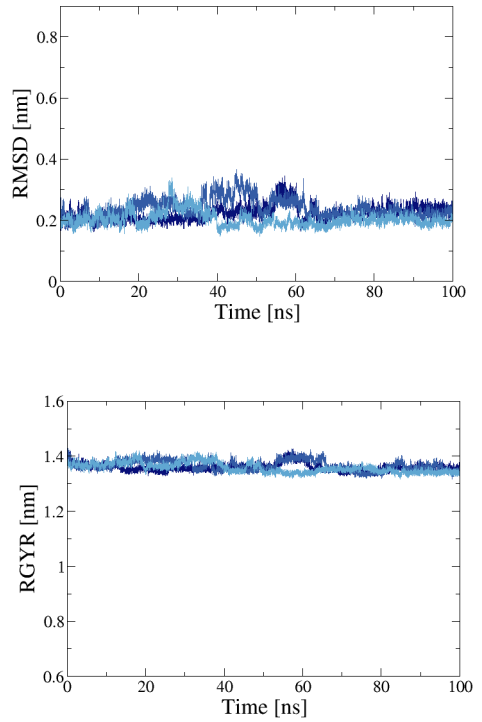

Figure S7: Time series of the backbone atom-positional root-mean-square deviation (RMSD) (top) and the radius of gyration (RGYR) (bottom) of the protein in AT water (black, left), CG water (red, middle), and AT/CG water with layer scheme 2 (blue, right). The replicas are shown in color gradients.
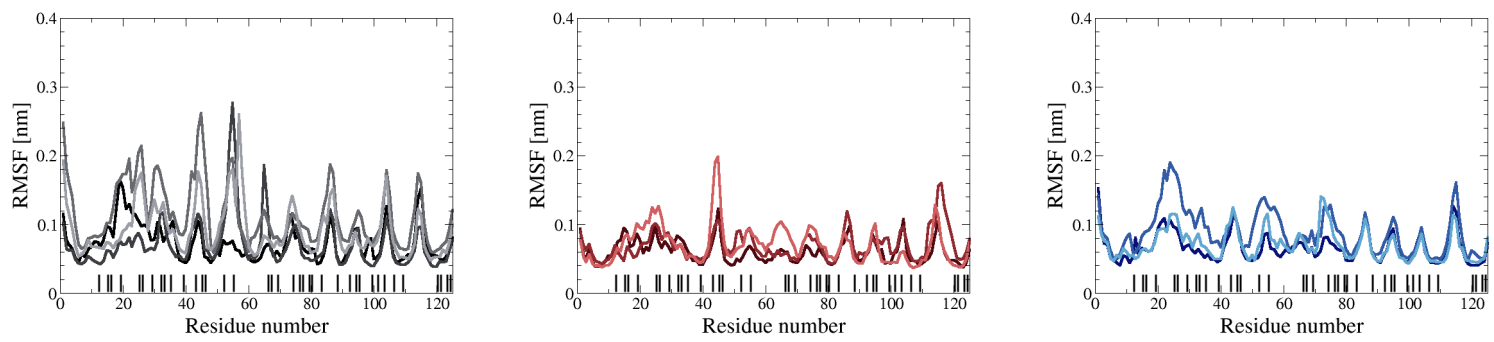

Figure S8: Root-mean-square fluctuation (RMSF) of the $C_{\alpha}$ atoms of the protein in AT water (black, left), CG water (red, middle), and AT/CG water with layer scheme 2 (blue, right). The replicas are shown in color gradients. The positions of the charged residues are indicated with a black dash on the $x$-axis. 
Full AT / Replica 1

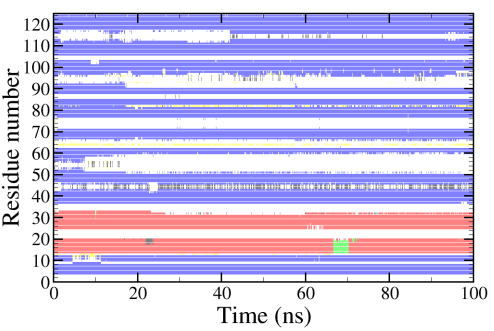

Full AT / Replica 2

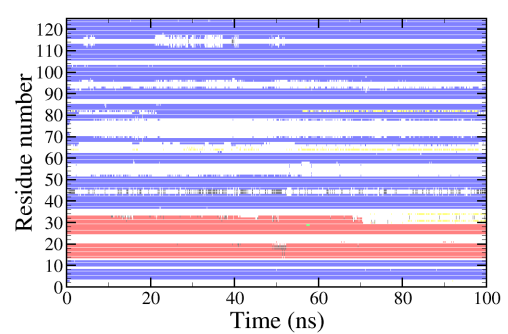

Full AT / Replica 4

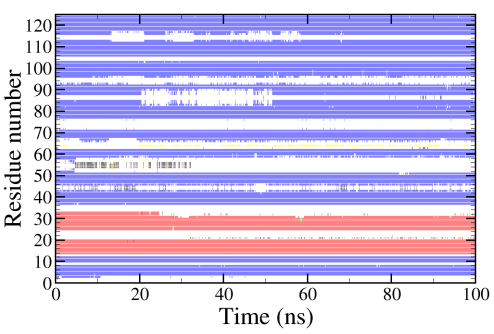

Full CG / Replica 1

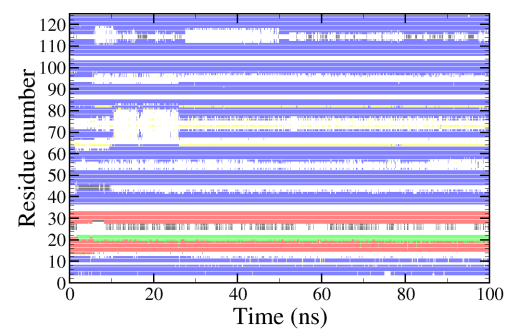

Layer Scheme 2 / Replica 1

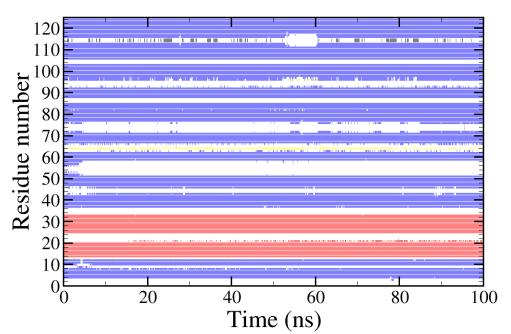

Full CG / Replica 2

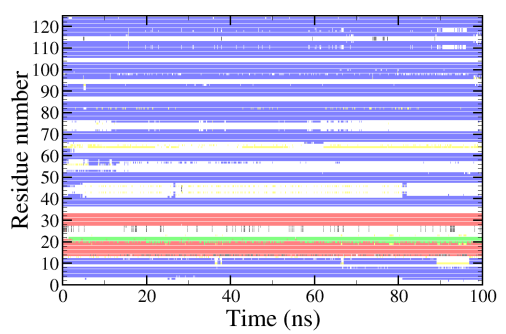

Layer Scheme 2 / Replica 2

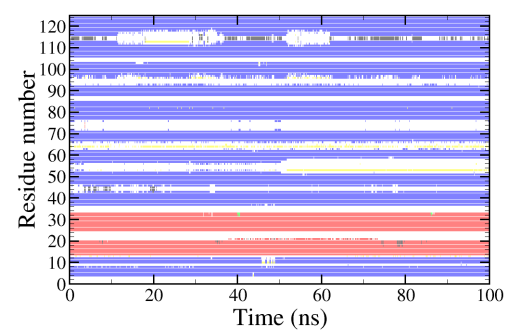

Full AT / Replica 3

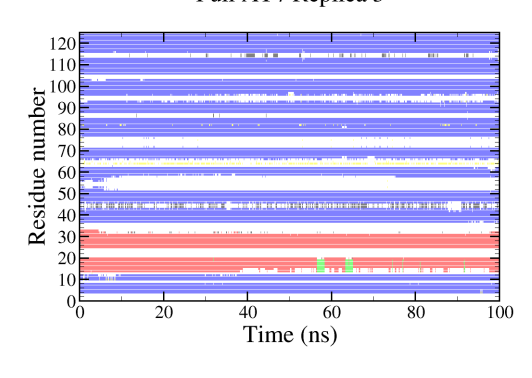

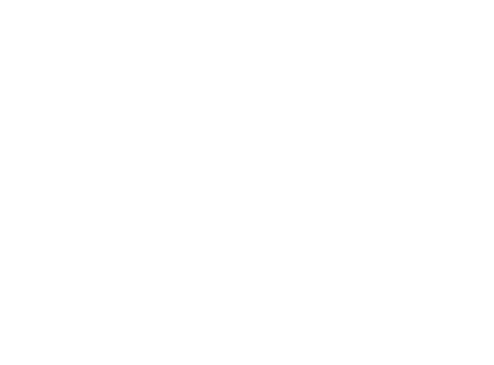

Full CG / Replica 3

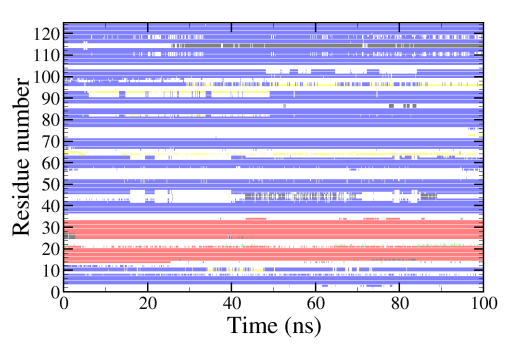

Layer Scheme 2 / Replica 3

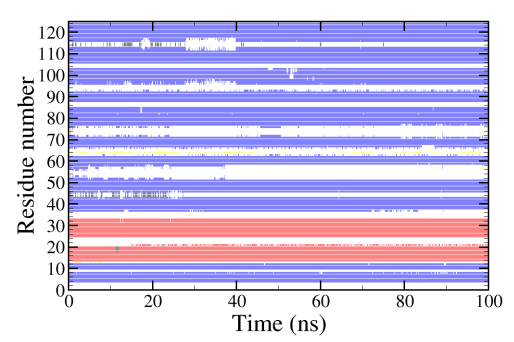

Figure S9: Time series of the secondary structure motifs of the protein in AT water (top), CG water (middle), and AT/CG water with layer scheme 2 (bottom). $\beta$-strands are shown in blue, $\beta$-bridges in yellow, $3_{10}$-helices in black, $\alpha$-helices in red, and $\pi$-helices in green. 


\subsection{Protein 1qk8}

Full AT
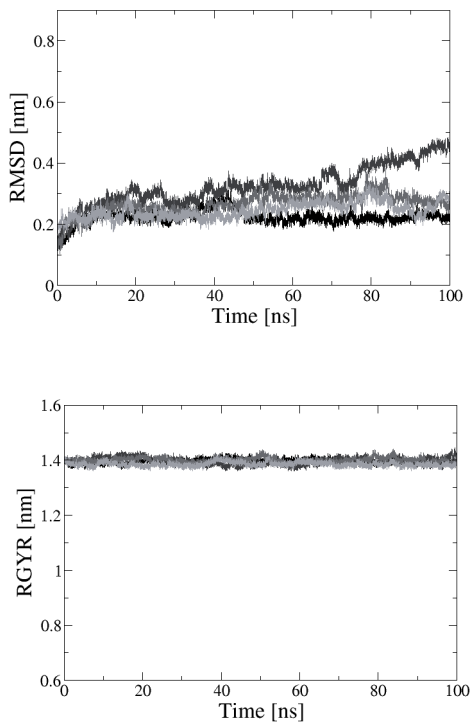

Full CG
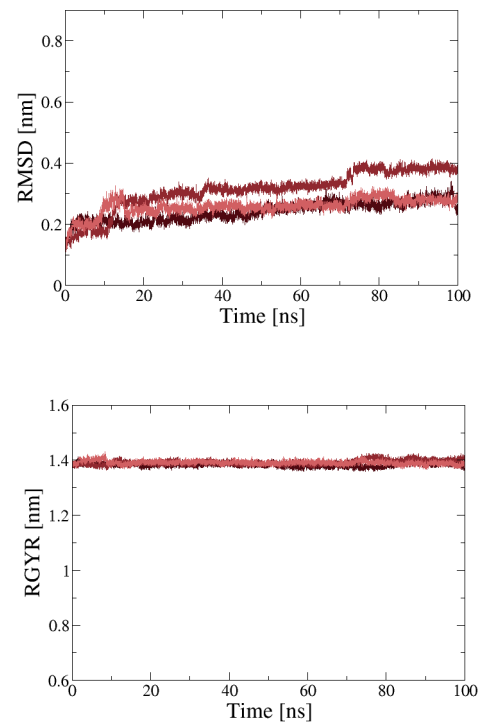

Layer Scheme 2
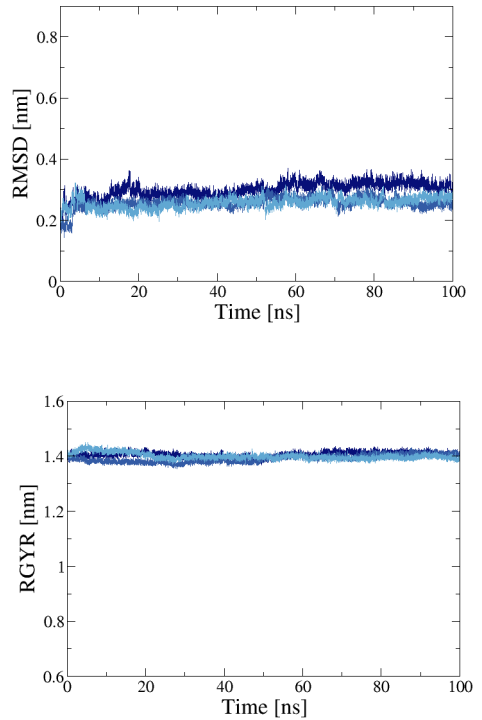

Figure S10: Time series of the backbone atom-positional root-mean-square deviation (RMSD) (top) and the radius of gyration (RGYR) (bottom) of the protein in AT water (black, left), CG water (red, middle), and AT/CG water with layer scheme 2 (blue, right). The replicas are shown in color gradients.
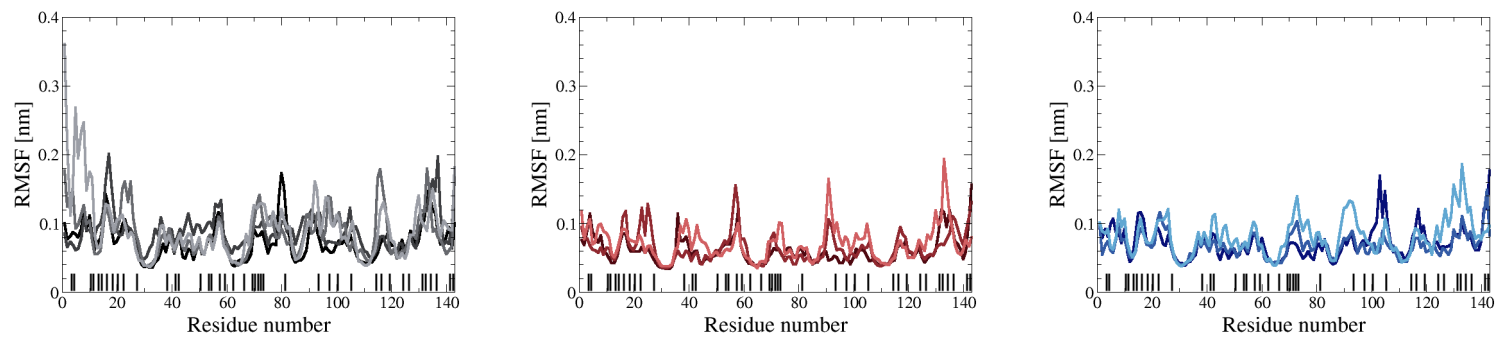

Figure S11: Root-mean-square fluctuation (RMSF) of the $C_{\alpha}$ atoms of the protein in AT water (black, left), CG water (red, middle), and AT/CG water with layer scheme 2 (blue, right). The replicas are shown in color gradients. The positions of the charged residues are indicated with a black dash on the $x$-axis. 
Full AT / Replica 1

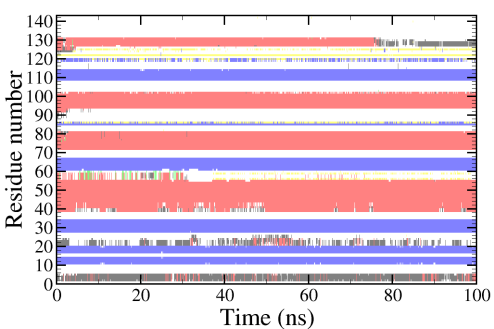

Full AT / Replica 2

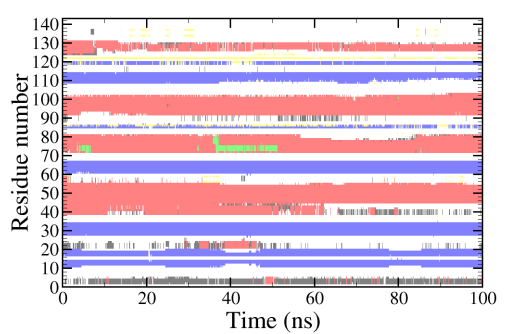

Full AT / Replica 4

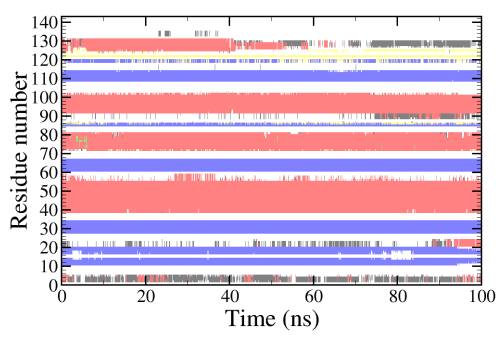

Full CG / Replica 1

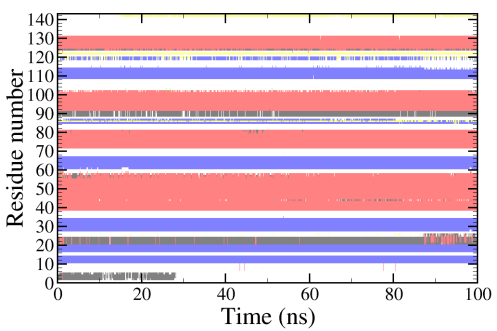

Layer Scheme 2 / Replica 1

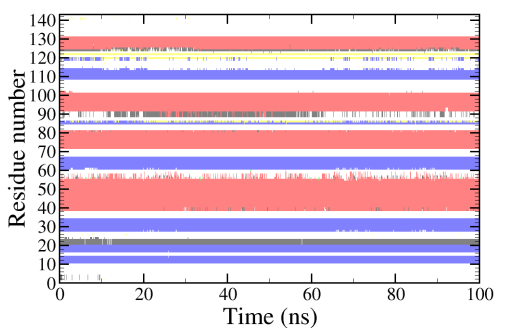

Full CG / Replica 2

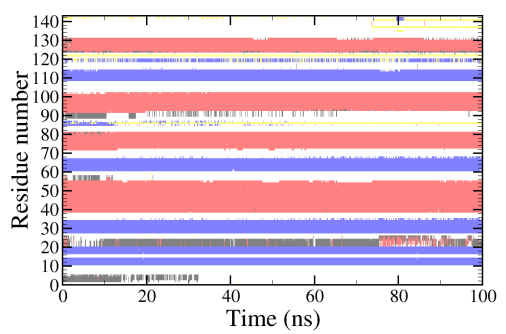

Layer Scheme 2 / Replica 2

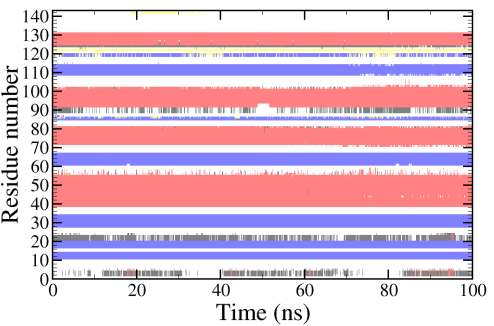

Full AT / Replica 3

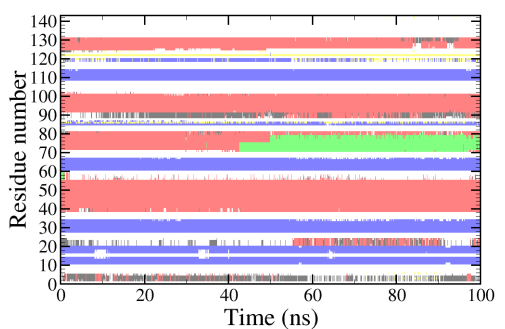

Full CG / Replica 3

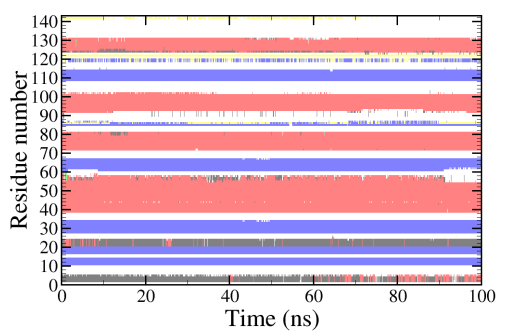

Layer Scheme 2 / Replica 3

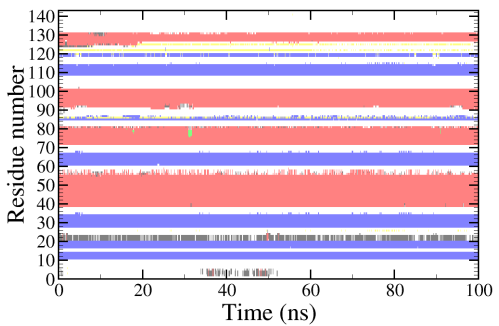

Figure S12: Time series of the secondary structure motifs of the protein in AT water (top), CG water (middle), and AT/CG water with layer scheme 2 (bottom). $\beta$-strands are shown in blue, $\beta$-bridges in yellow, $3_{10}$-helices in black, $\alpha$-helices in red, and $\pi$-helices in green. 


\subsection{Protein 1qqv}

Full AT
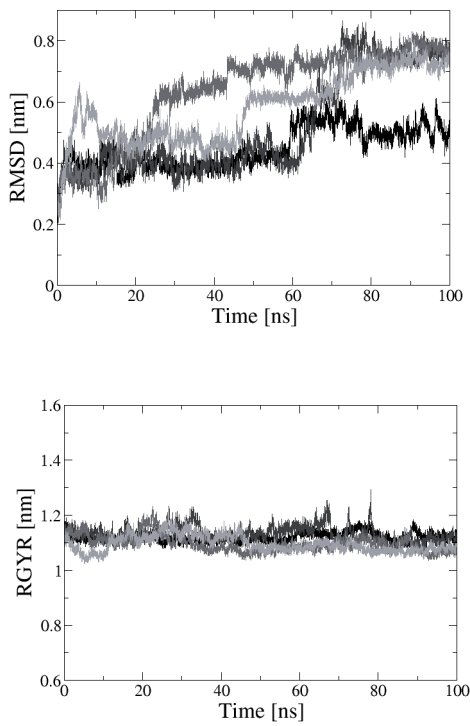

Full CG
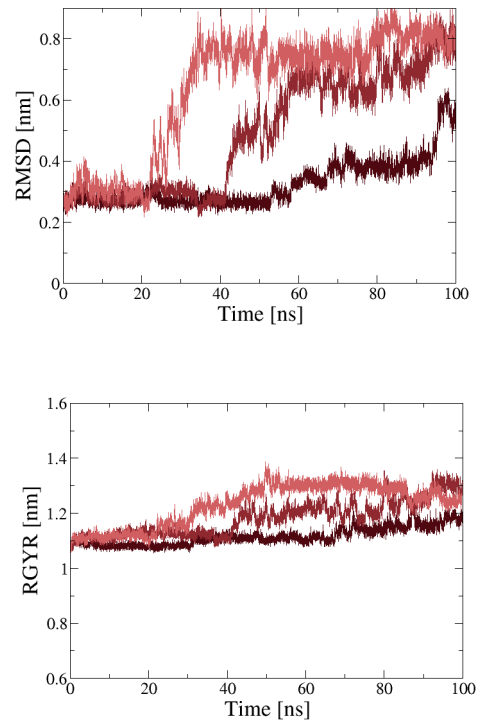

Layer Scheme 2
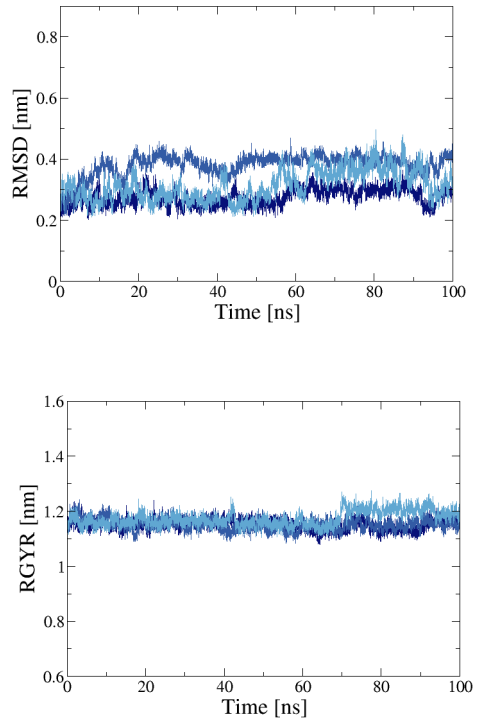

Figure S13: Time series of the backbone atom-positional root-mean-square deviation (RMSD) (top) and the radius of gyration (RGYR) (bottom) of the protein in AT water (black, left), CG water (red, middle), and AT/CG water with layer scheme 2 (blue, right). The replicas are shown in color gradients.
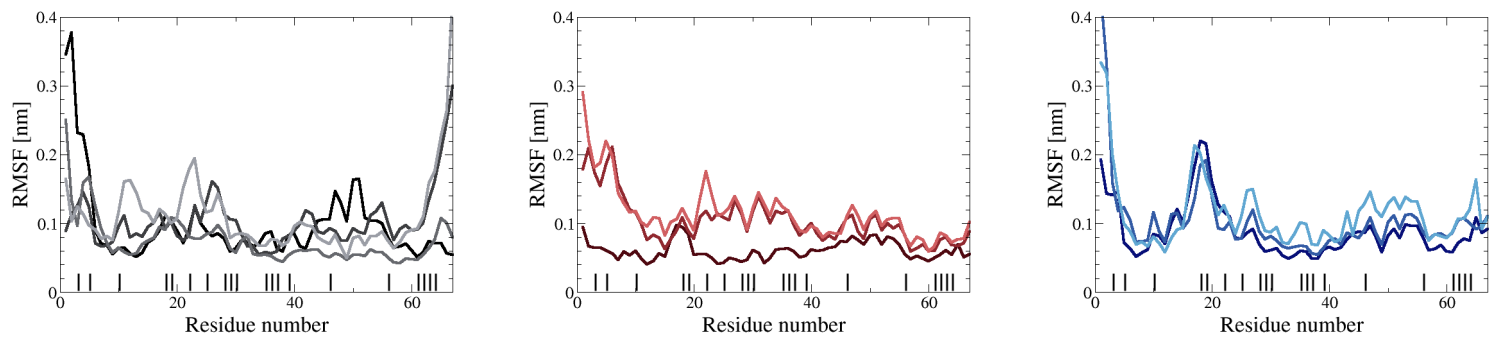

Figure S14: Root-mean-square fluctuation (RMSF) of the $C_{\alpha}$ atoms of the protein in AT water (black, left), CG water (red, middle), and AT/CG water with layer scheme 2 (blue, right). The replicas are shown in color gradients. The positions of the charged residues are indicated with a black dash on the $x$-axis. 
Full AT / Replica 1

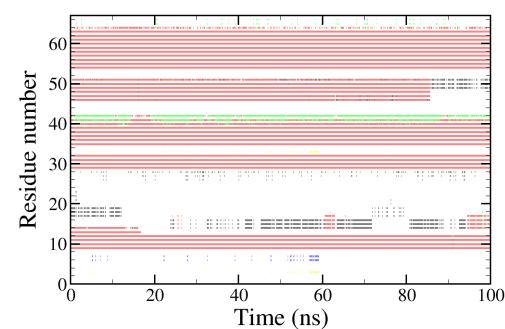

Full AT / Replica 2

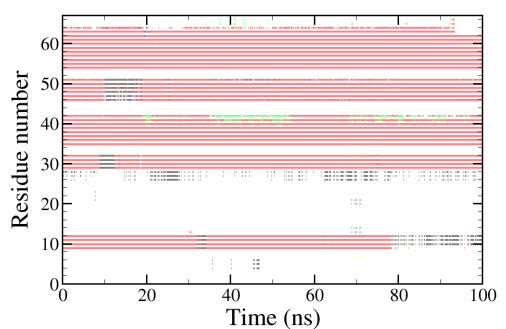

Full AT / Replica 4

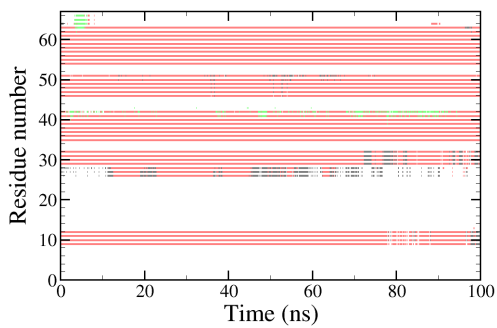

Full CG / Replica 1

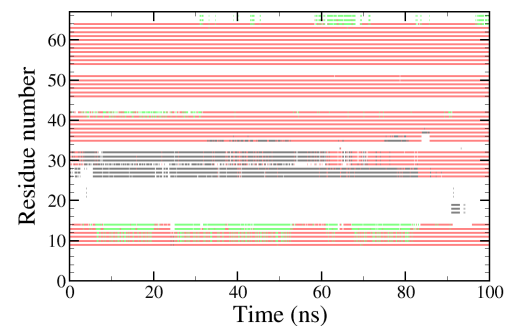

Layer Scheme 2 / Replica 1

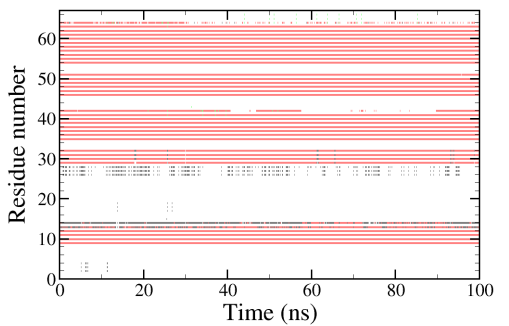

Full CG / Replica 2

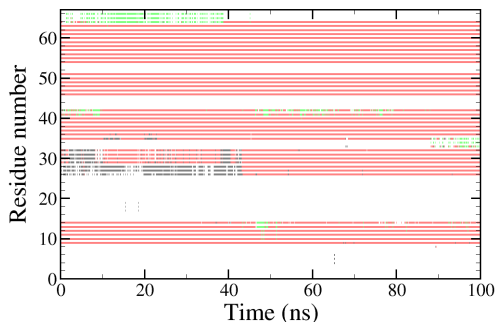

Layer Scheme 2 / Replica 2

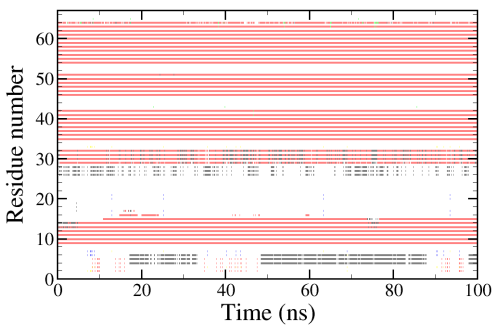

Full AT / Replica 3

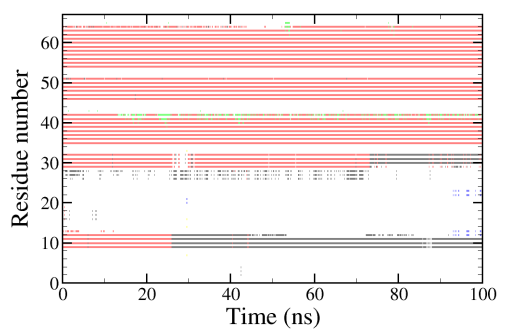

Full CG / Replica 3

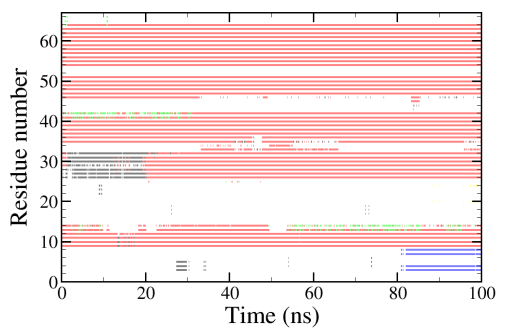

Layer Scheme 2 / Replica 3

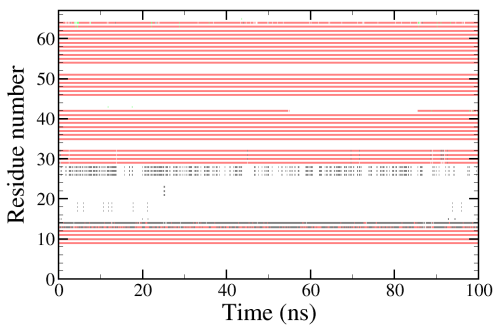

Figure S15: Time series of the secondary structure motifs of the protein in AT water (top), CG water (middle), and AT/CG water with layer scheme 2 (bottom). $\beta$-strands are shown in blue, $\beta$-bridges in yellow, $3_{10}$-helices in black, $\alpha$-helices in red, and $\pi$-helices in green. 


\subsection{Protein 1tvq}

Full AT
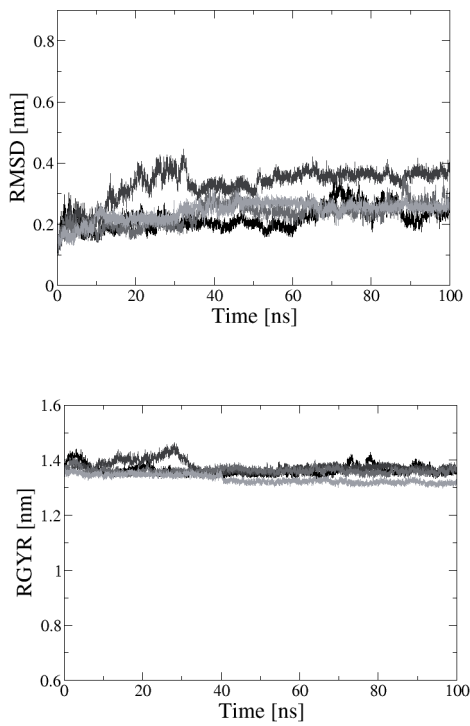

Full CG
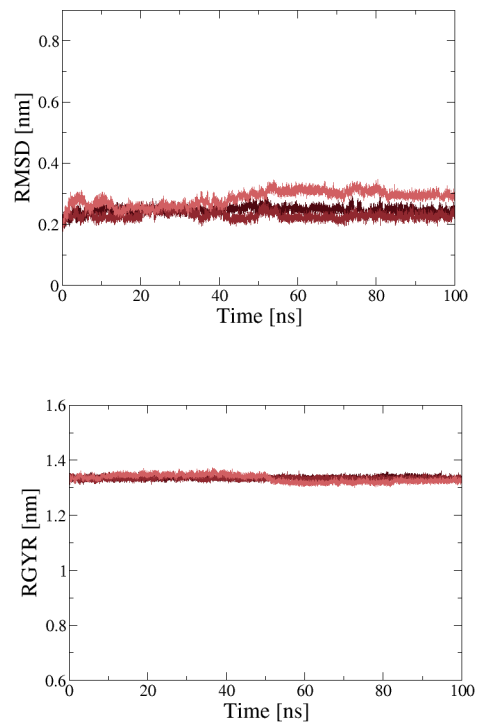

Layer Scheme 2
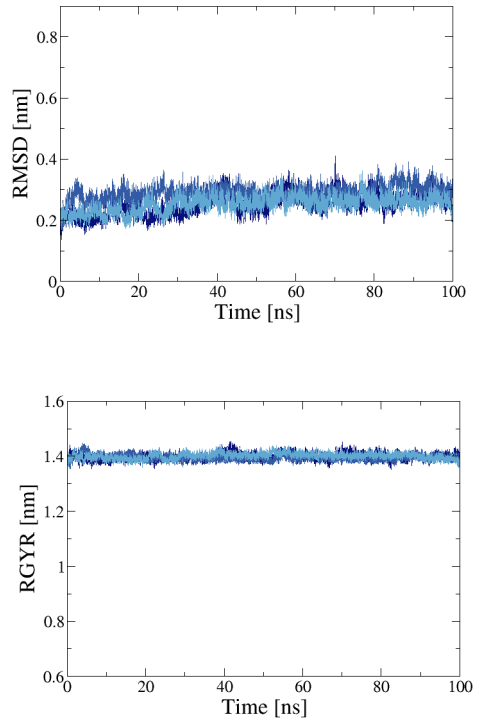

Figure S16: Time series of the backbone atom-positional root-mean-square deviation (RMSD) (top) and the radius of gyration (RGYR) (bottom) of the protein in AT water (black, left), CG water (red, middle), and AT/CG water with layer scheme 2 (blue, right). The replicas are shown in color gradients.
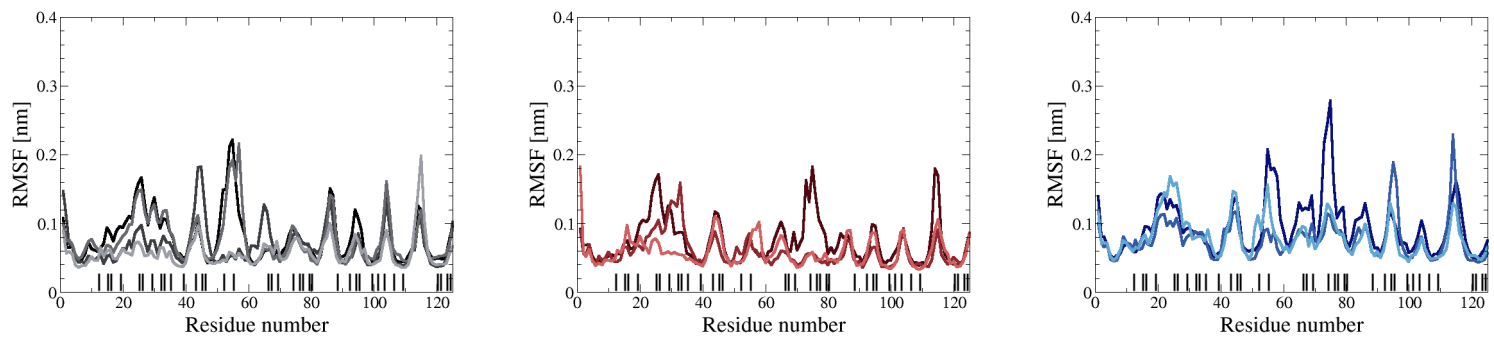

Figure S17: Root-mean-square fluctuation (RMSF) of the $C_{\alpha}$ atoms of the protein in AT water (black, left), CG water (red, middle), and AT/CG water with layer scheme 2 (blue, right). The replicas are shown in color gradients. The positions of the charged residues are indicated with a black dash on the $x$-axis. 
Full AT / Replica 1

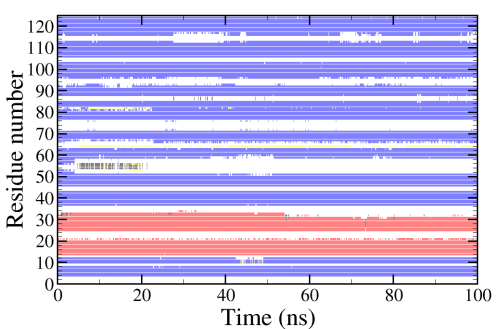

Full AT / Replica 2

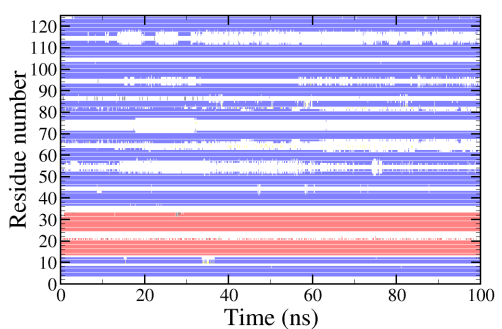

Full AT / Replica 4

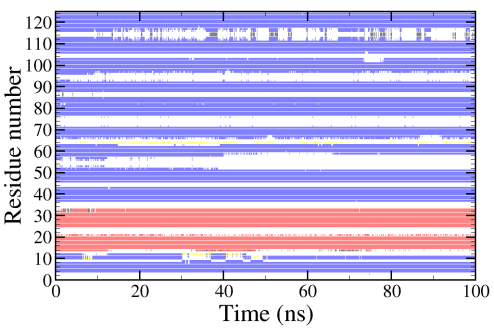

Full CG / Replica 1

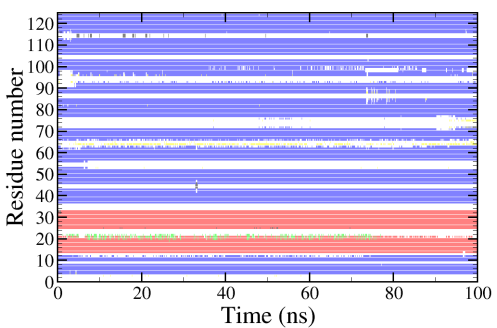

Layer Scheme 2 / Replica 1

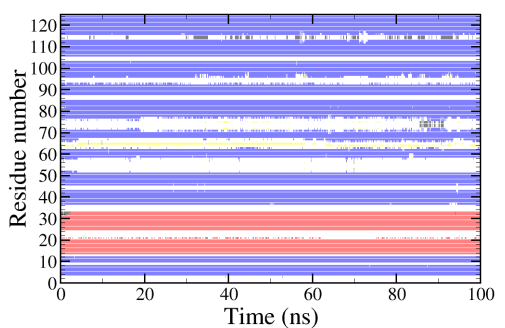

Full CG / Replica 2

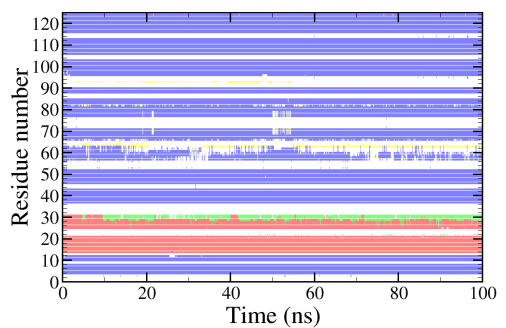

Layer Scheme 2 / Replica 2

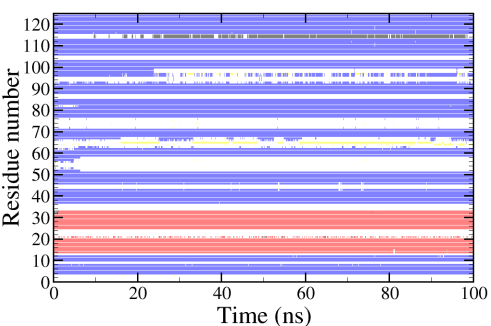

Full AT / Replica 3

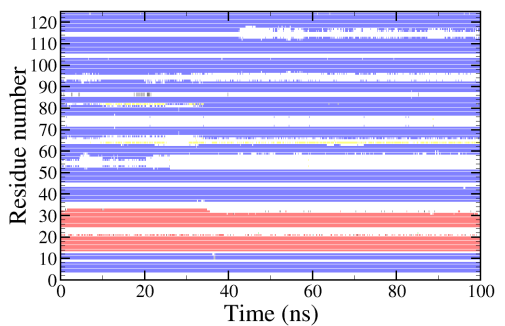

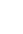




\subsection{Protein 1ucs}

Full AT
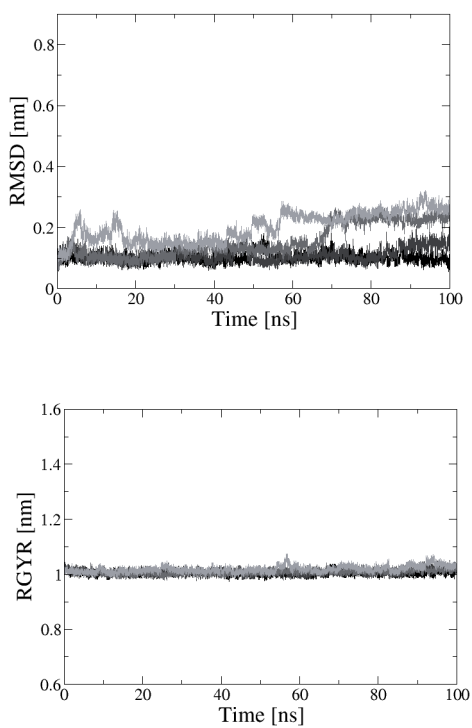

Full CG
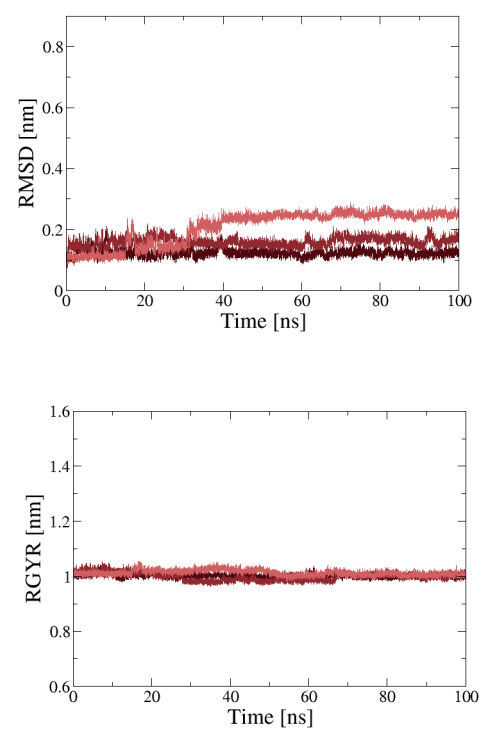

Layer Scheme 2
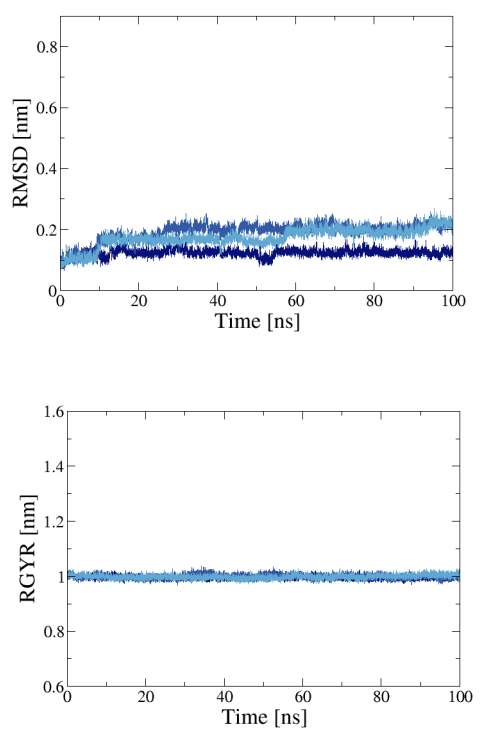

Figure S19: Time series of the backbone atom-positional root-mean-square deviation (RMSD) (top) and the radius of gyration (RGYR) (bottom) of the protein in AT water (black, left), CG water (red, middle), and AT/CG water with layer scheme 2 (blue, right). The replicas are shown in color gradients.
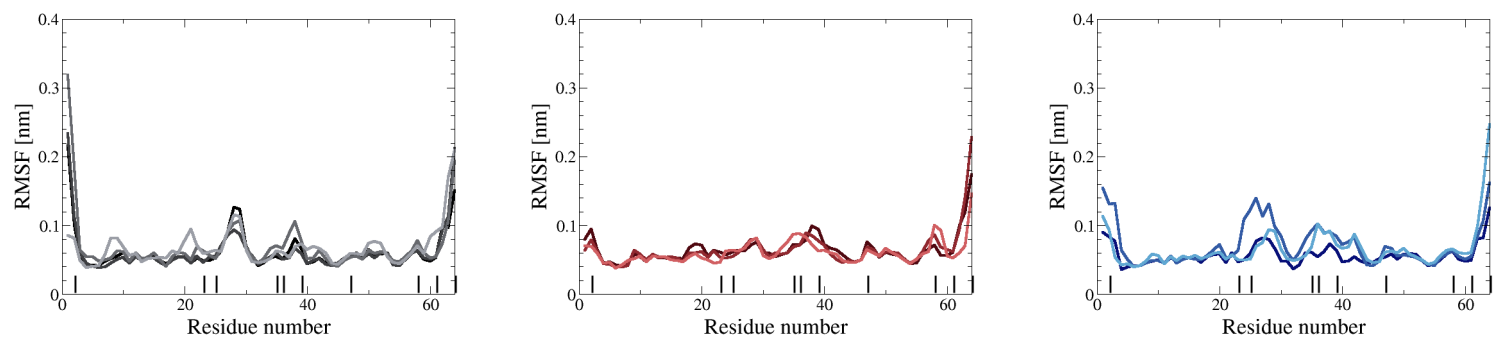

Figure S20: Root-mean-square fluctuation (RMSF) of the $C_{\alpha}$ atoms of the protein in AT water (black, left), CG water (red, middle), and AT/CG water with layer scheme 2 (blue, right). The replicas are shown in color gradients. The positions of the charged residues are indicated with a black dash on the $x$-axis. 
Full AT / Replica 1

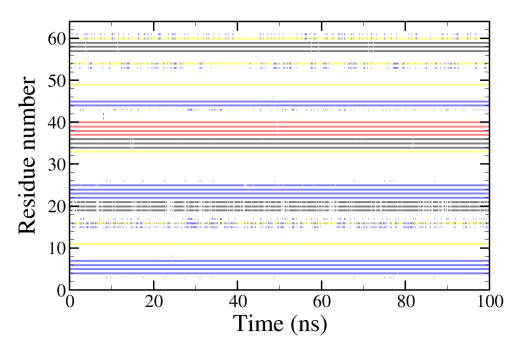

Full AT / Replica 2

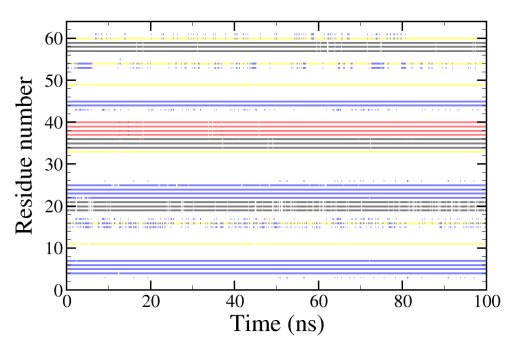

Full AT / Replica 4

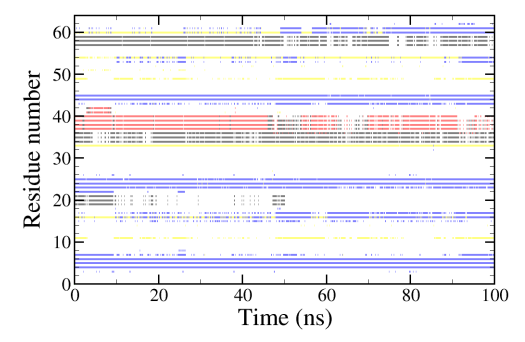

Full CG / Replica 1

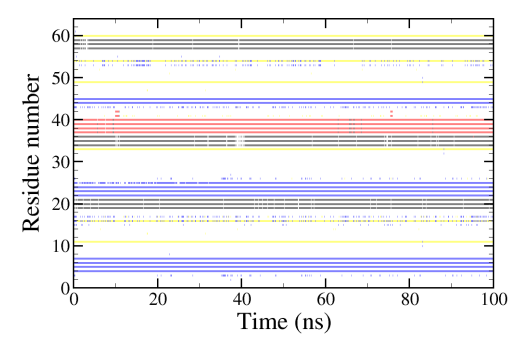

Layer Scheme 2 / Replica 1

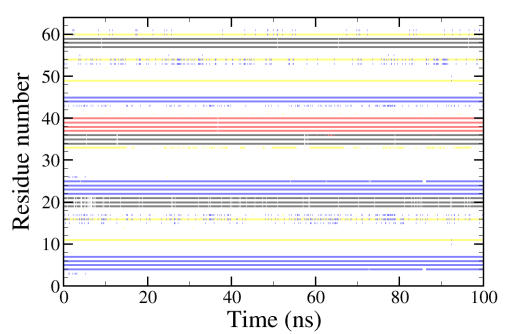

Full CG / Replica 2

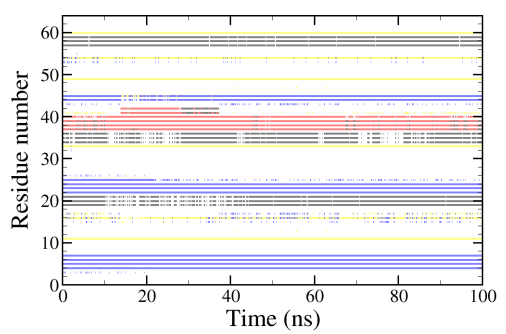

Layer Scheme 2 / Replica 2

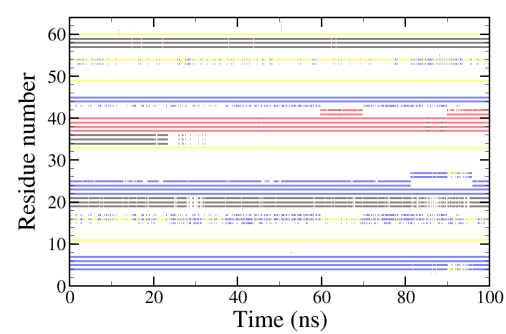

Full AT / Replica 3

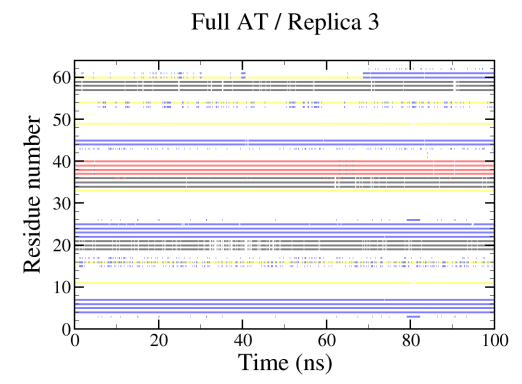

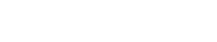

$$
\text { . }
$$




\subsection{Protein 1uxz}

Full AT
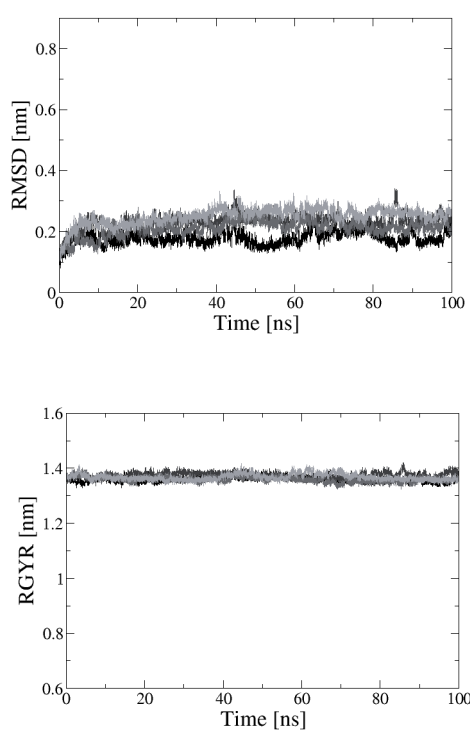

Full CG
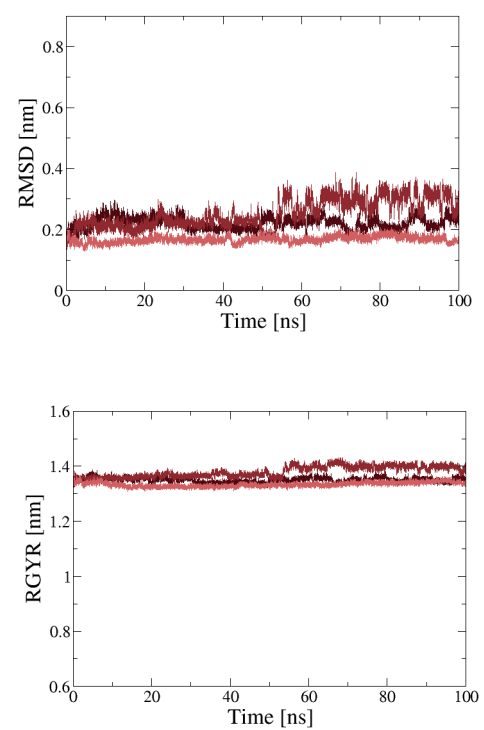

Layer Scheme 2
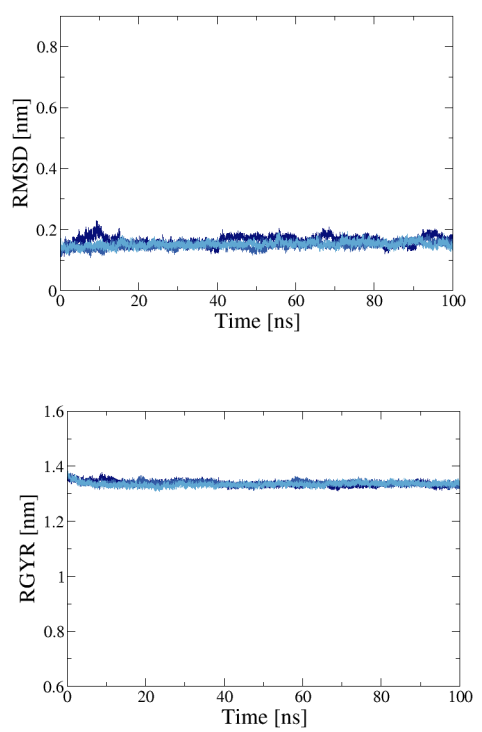

Figure S22: Time series of the backbone atom-positional root-mean-square deviation (RMSD) (top) and the radius of gyration (RGYR) (bottom) of the protein in AT water (black, left), CG water (red, middle), and AT/CG water with layer scheme 2 (blue, right). The replicas are shown in color gradients.
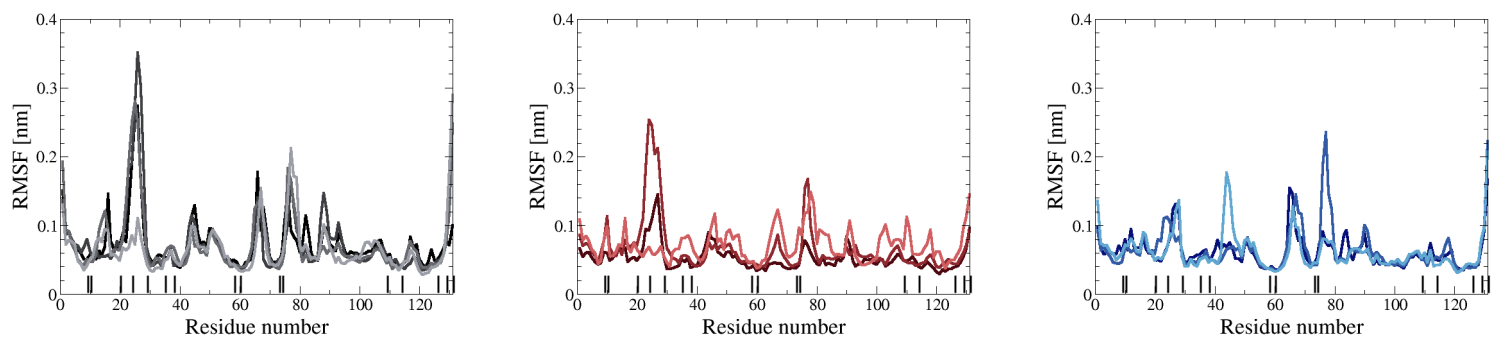

Figure S23: Root-mean-square fluctuation (RMSF) of the $C_{\alpha}$ atoms of the protein in AT water (black, left), CG water (red, middle), and AT/CG water with layer scheme 2 (blue, right). The replicas are shown in color gradients. The positions of the charged residues are indicated with a black dash on the $x$-axis. 
Full AT / Replica

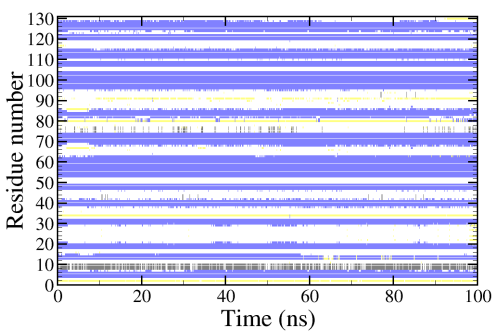

Full AT / Replica 2

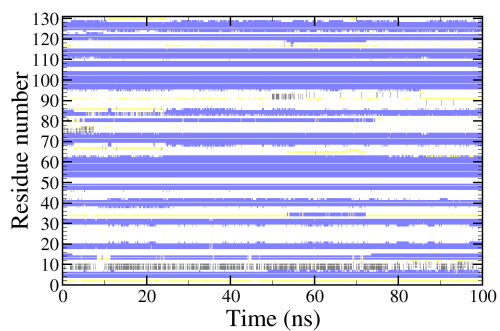

Full AT / Replica 4

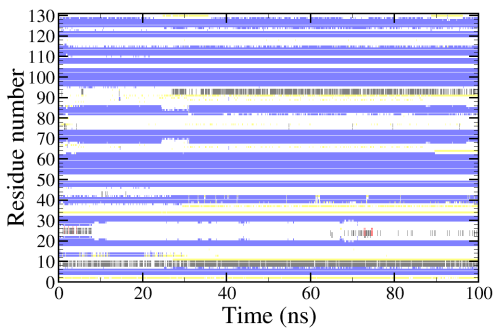

Full CG / Replica 1

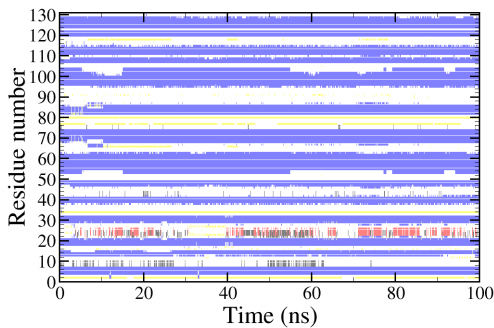

Layer Scheme 2 / Replica 1

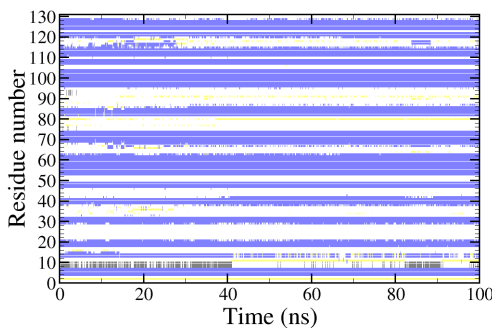

Full CG / Replica 2

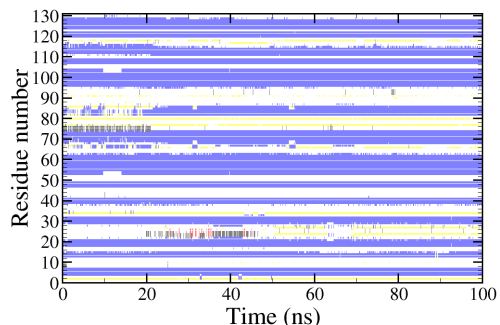

Layer Scheme 2 / Replica 2

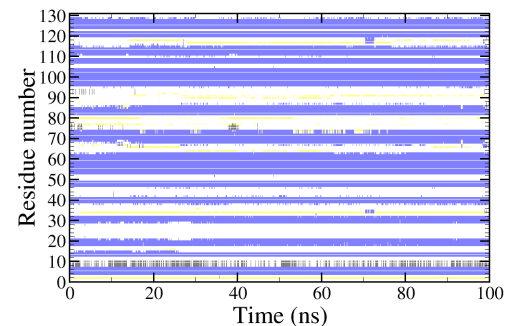

Full AT / Replica 3

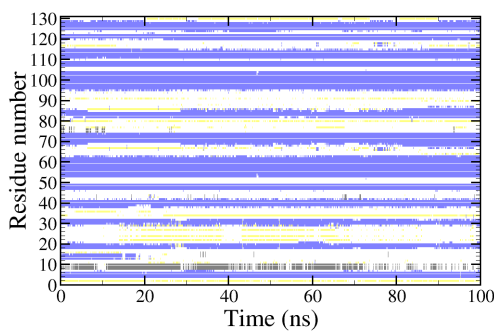

Time (ns)

Figure S24: Time series of the secondary structure motifs of the protein in AT water (top), CG water (middle), and AT/CG water with layer scheme 2 (bottom). $\beta$-strands are shown in blue, $\beta$-bridges in yellow, $3_{10}$-helices in black, $\alpha$-helices in red, and $\pi$-helices in green. 


\subsection{Protein 1zvg}

Full AT
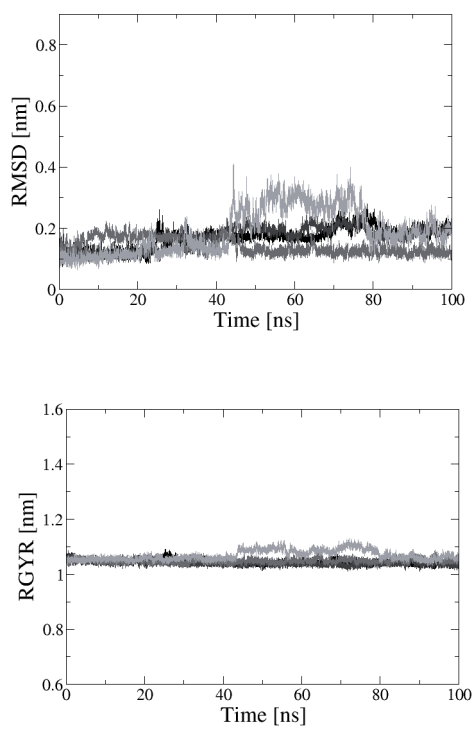

Full CG
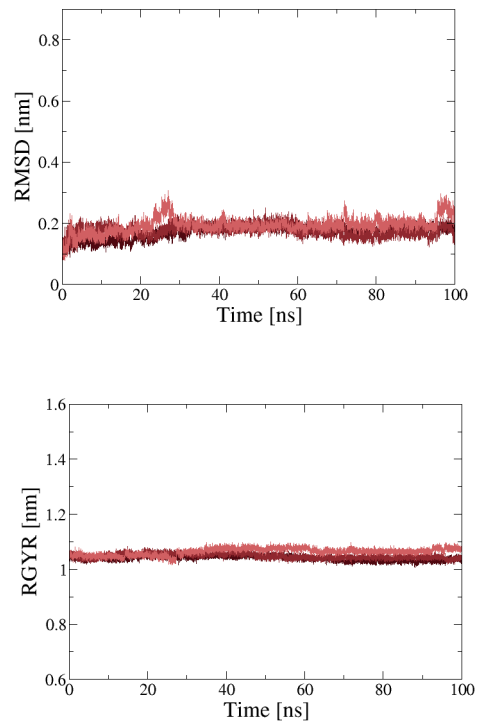

Layer Scheme 2
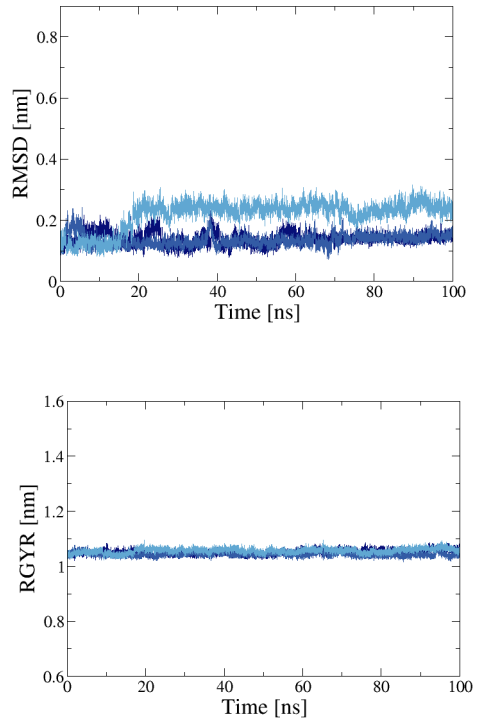

Figure S25: Time series of the backbone atom-positional root-mean-square deviation (RMSD) (top) and the radius of gyration (RGYR) (bottom) of the protein in AT water (black, left), CG water (red, middle), and AT/CG water with layer scheme 2 (blue, right). The replicas are shown in color gradients.
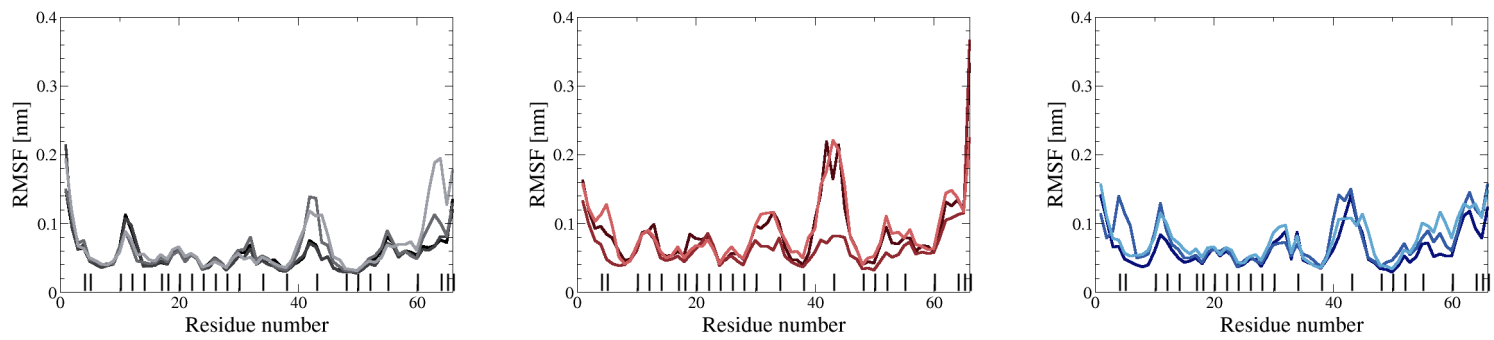

Figure S26: Root-mean-square fluctuation (RMSF) of the $C_{\alpha}$ atoms of the protein in AT water (black, left), CG water (red, middle), and AT/CG water with layer scheme 2 (blue, right). The replicas are shown in color gradients. The positions of the charged residues are indicated with a black dash on the $x$-axis. 
Full AT / Replica

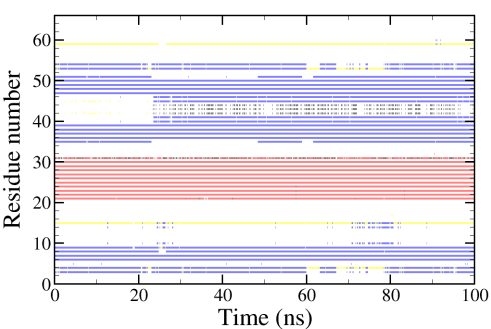

Full AT / Replica 2

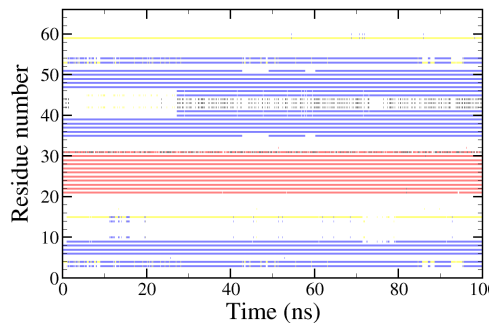

Full AT / Replica 4

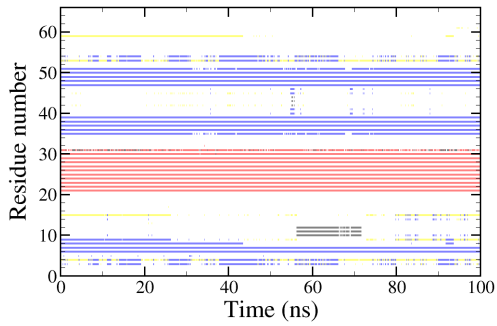

Full CG / Replica 1

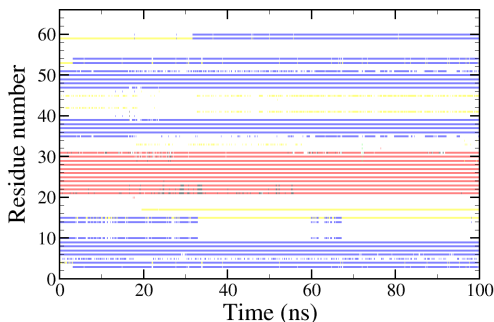

Layer Scheme 2 / Replica 1

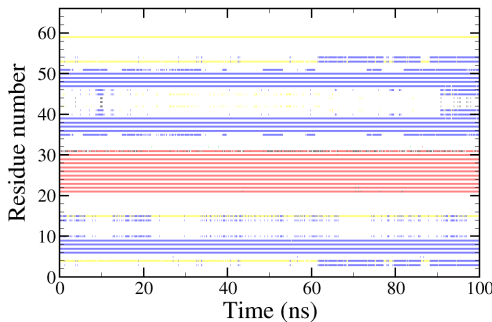

Full CG / Replica 2

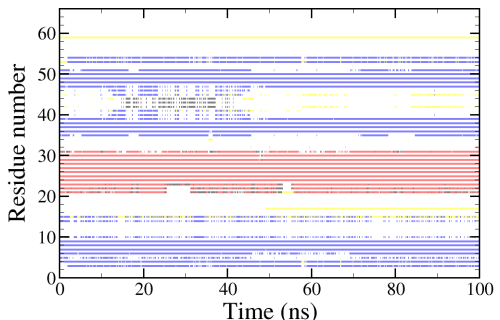

Layer Scheme 2 / Replica 2

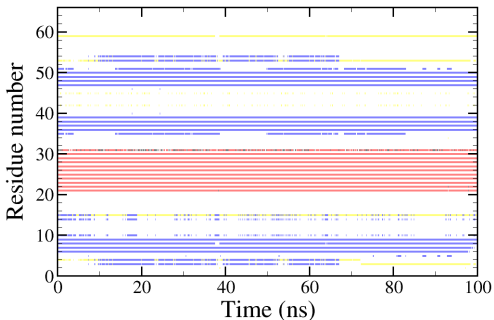

Full AT / Replica 3

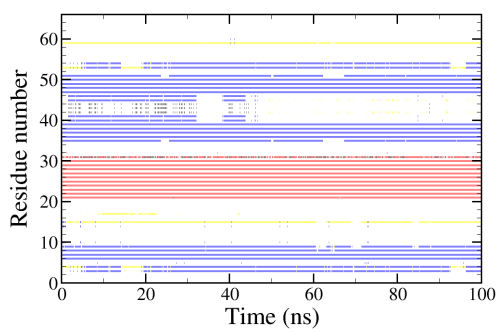

Time (ns)

Figure S27: Time series of the secondary structure motifs of the protein in AT water (top), CG water (middle), and AT/CG water with layer scheme 2 (bottom). $\beta$-strands are shown in blue, $\beta$-bridges in yellow, $3_{10}$-helices in black, $\alpha$-helices in red, and $\pi$-helices in green. 


\subsection{Protein 2cwr}

Full AT
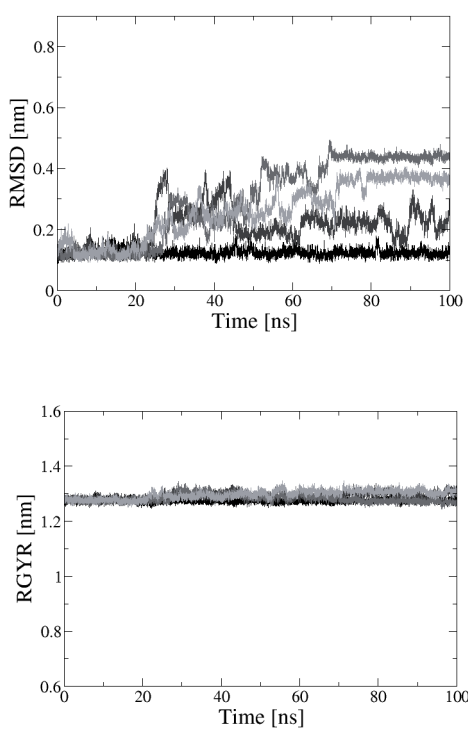

Full CG
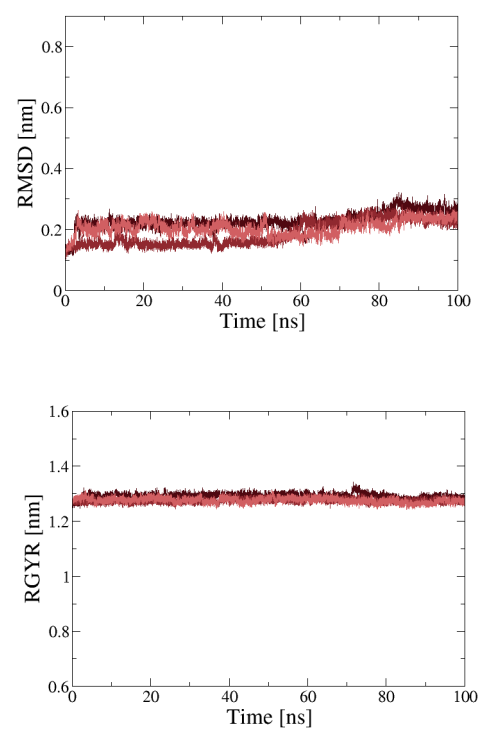

Layer Scheme 2
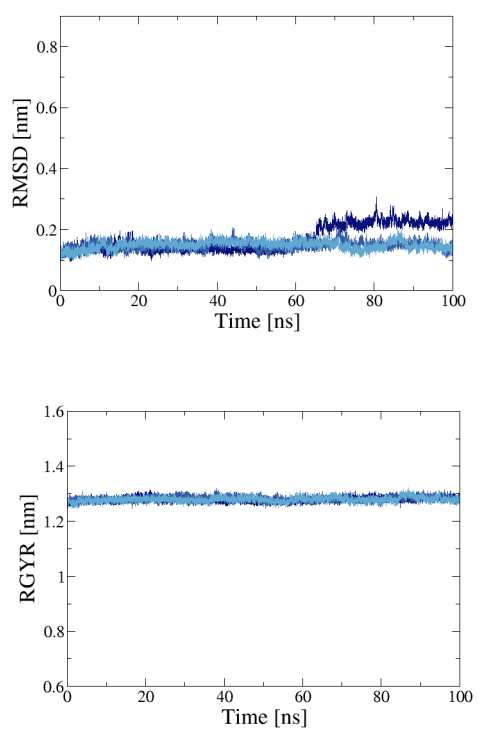

Figure S28: Time series of the backbone atom-positional root-mean-square deviation (RMSD) (top) and the radius of gyration (RGYR) (bottom) of the protein in AT water (black, left), CG water (red, middle), and AT/CG water with layer scheme 2 (blue, right). The replicas are shown in color gradients.
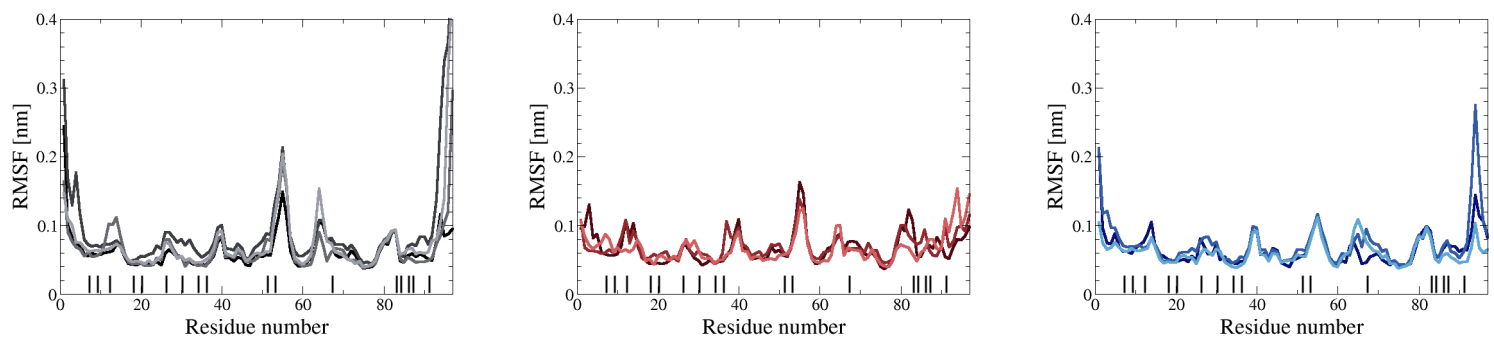

Figure S29: Root-mean-square fluctuation (RMSF) of the $C_{\alpha}$ atoms of the protein in AT water (black, left), CG water (red, middle), and AT/CG water with layer scheme 2 (blue, right). The replicas are shown in color gradients. The positions of the charged residues are indicated with a black dash on the $x$-axis. 
Full AT / Replica 1

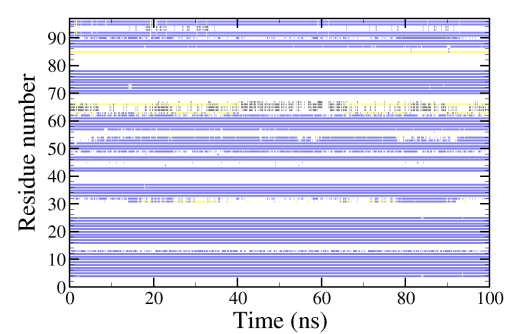

Full AT / Replica 2

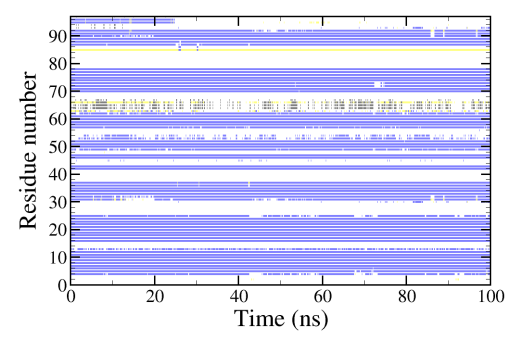

Full AT / Replica 4

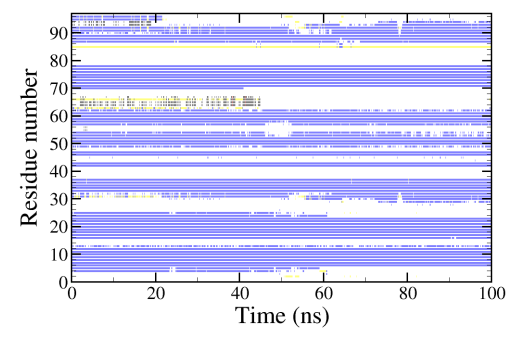

Full CG / Replica 1

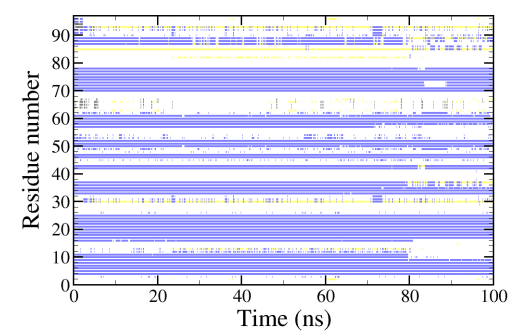

Layer Scheme 2 / Replica 1

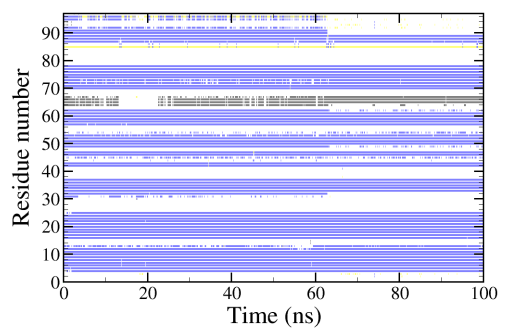

Full CG / Replica 2

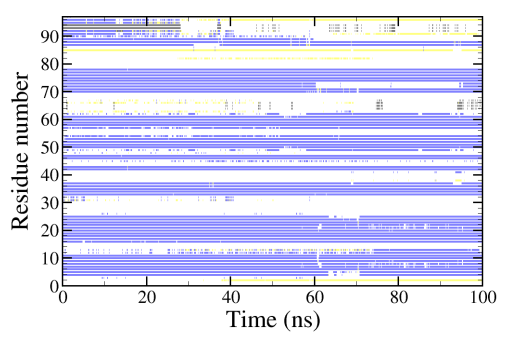

Layer Scheme 2 / Replica 2

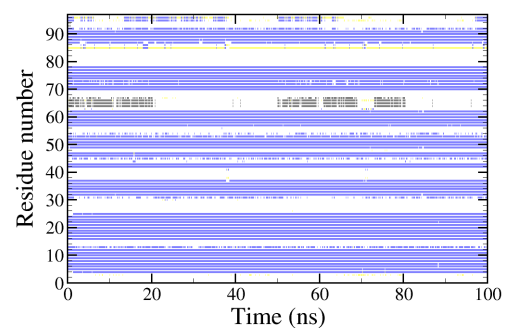

Full AT / Replica 3

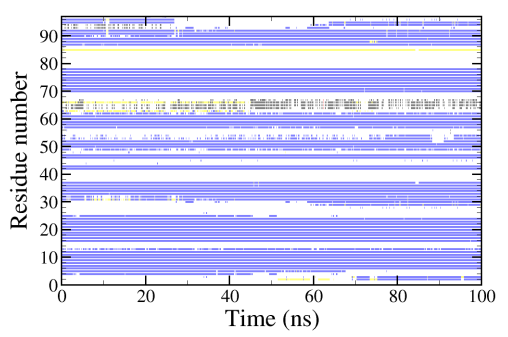




\subsection{Protein 2czn}

Full AT
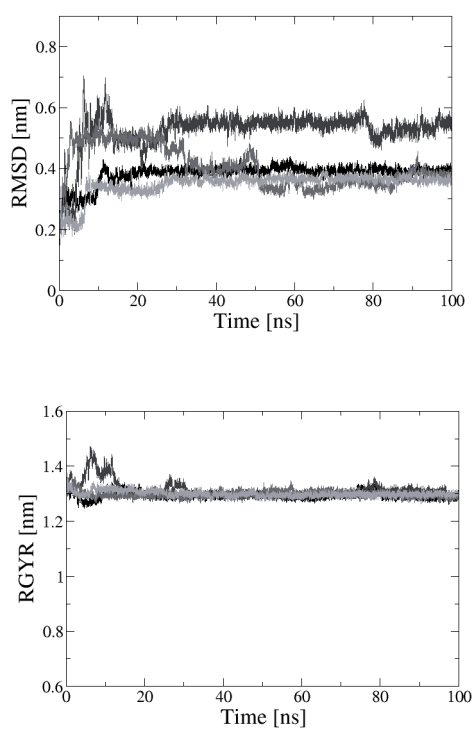

Full CG
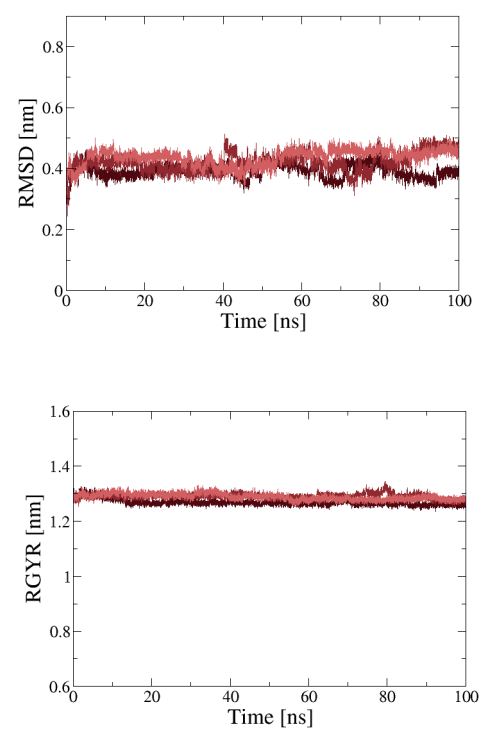

Layer Scheme 2
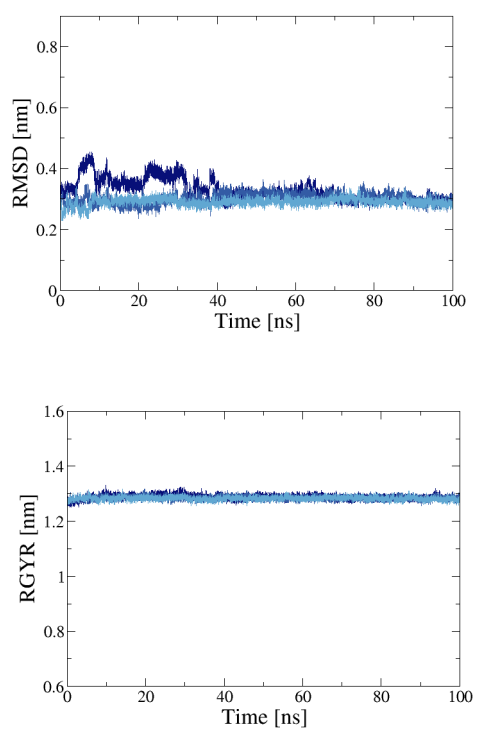

Figure S31: Time series of the backbone atom-positional root-mean-square deviation (RMSD) (top) and the radius of gyration (RGYR) (bottom) of the protein in AT water (black, left), CG water (red, middle), and AT/CG water with layer scheme 2 (blue, right). The replicas are shown in color gradients.
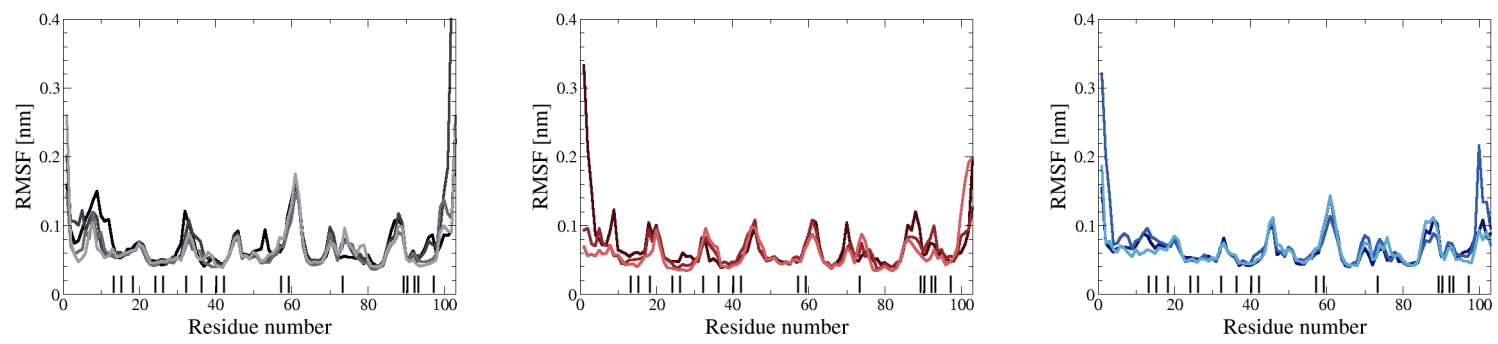

Figure S32: Root-mean-square fluctuation (RMSF) of the $C_{\alpha}$ atoms of the protein in AT water (black, left), CG water (red, middle), and AT/CG water with layer scheme 2 (blue, right). The replicas are shown in color gradients. The positions of the charged residues are indicated with a black dash on the $x$-axis. 
Full AT / Replica 1

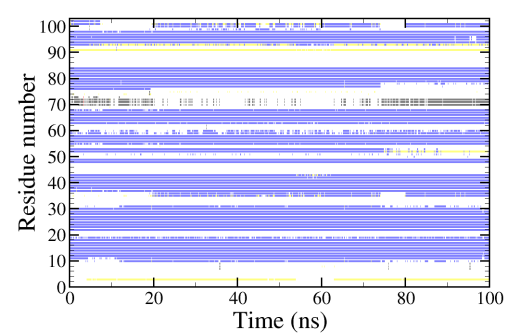

Full AT / Replica 2

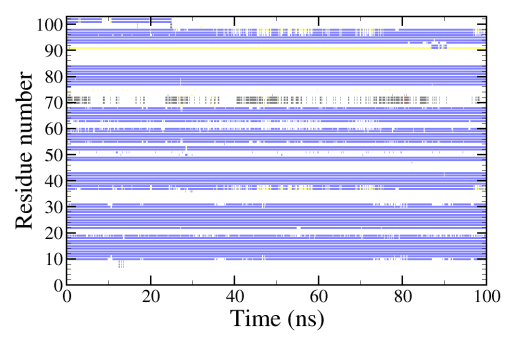

Full AT / Replica 4

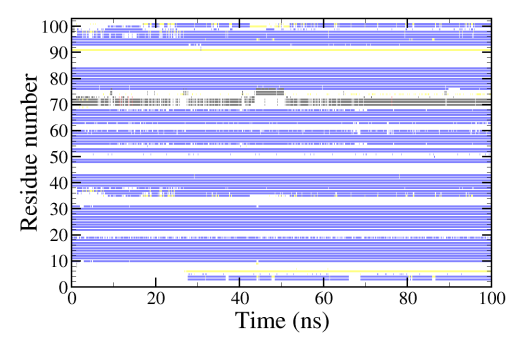

Full CG / Replica 1

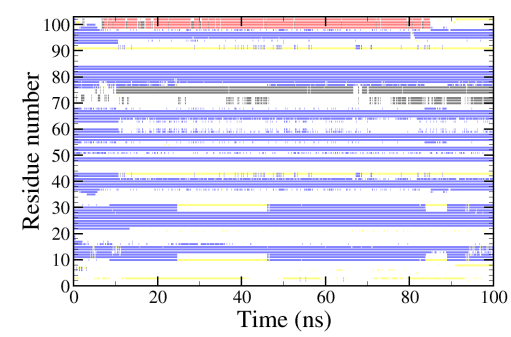

Layer Scheme 2 / Replica 1

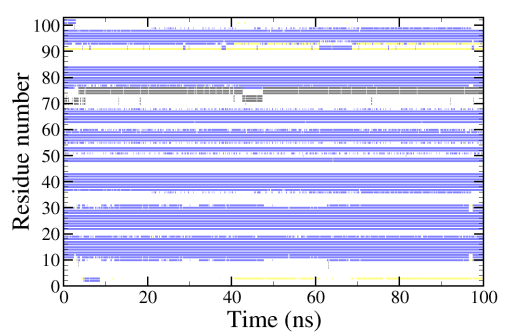

Full CG / Replica 2

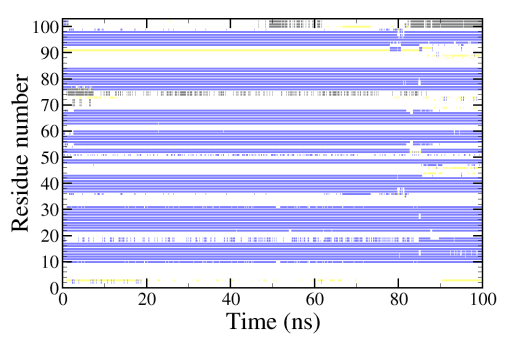

Layer Scheme 2 / Replica 2

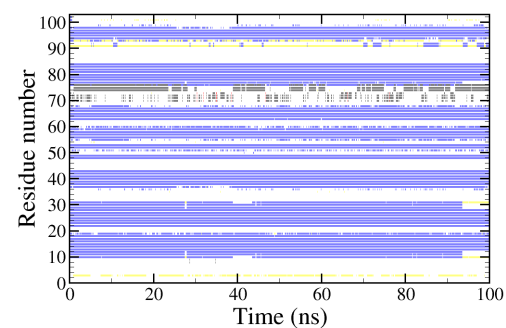

Full AT / Replica 3

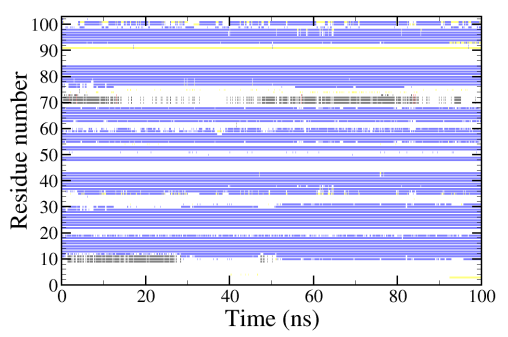




\subsection{Protein 2gkt}

Full AT
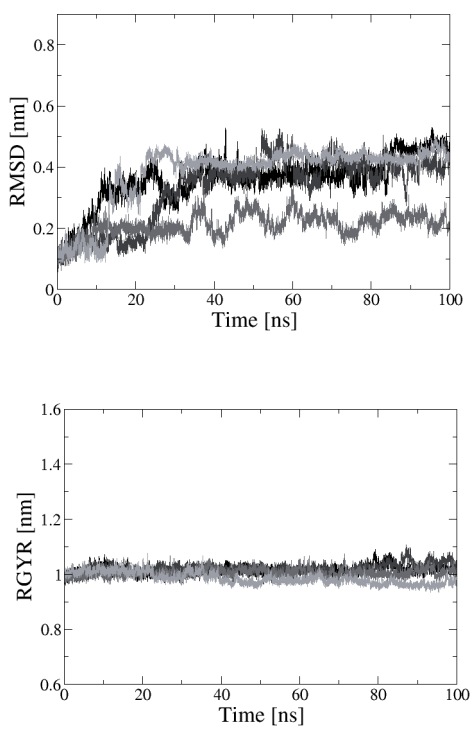

Full CG
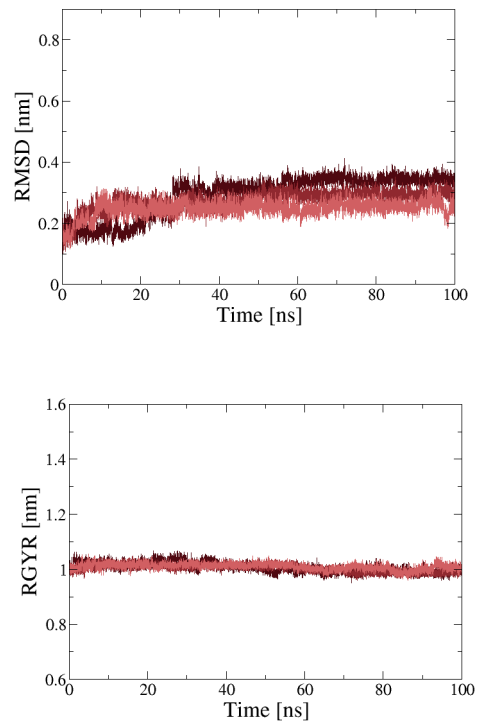

Layer Scheme 2
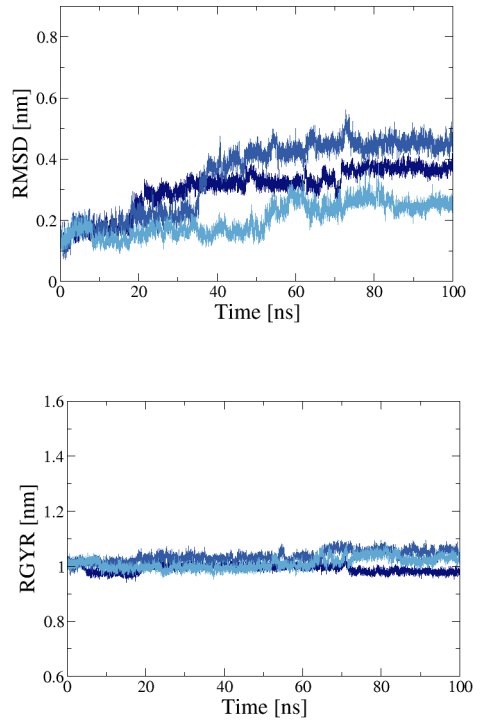

Figure S34: Time series of the backbone atom-positional root-mean-square deviation (RMSD) (top) and the radius of gyration (RGYR) (bottom) of the protein in AT water (black, left), CG water (red, middle), and AT/CG water with layer scheme 2 (blue, right). The replicas are shown in color gradients.
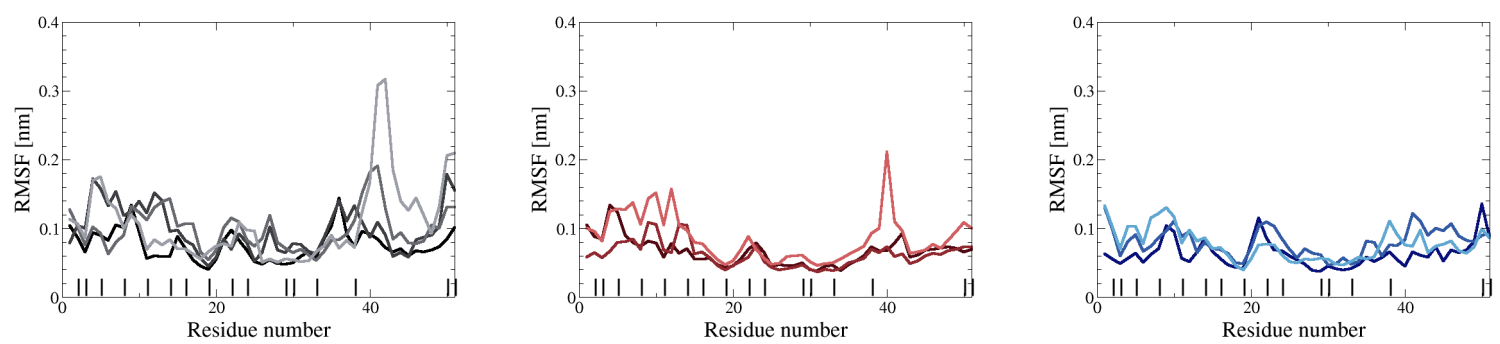

Figure S35: Root-mean-square fluctuation (RMSF) of the $C_{\alpha}$ atoms of the protein in AT water (black, left), CG water (red, middle), and AT/CG water with layer scheme 2 (blue, right). The replicas are shown in color gradients. The positions of the charged residues are indicated with a black dash on the $x$-axis. 
Full AT / Replica 1
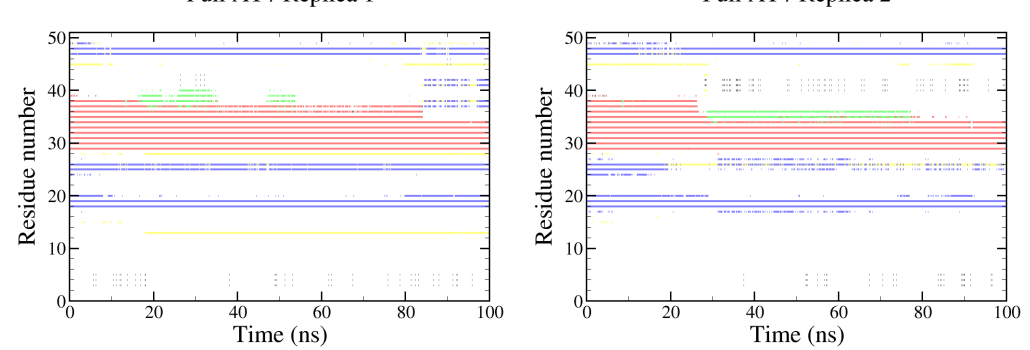

Full AT / Replica 4

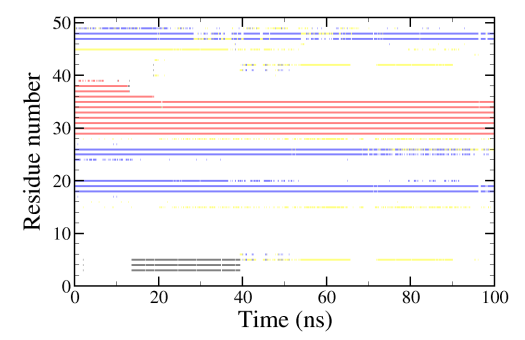

Full CG / Replica 1

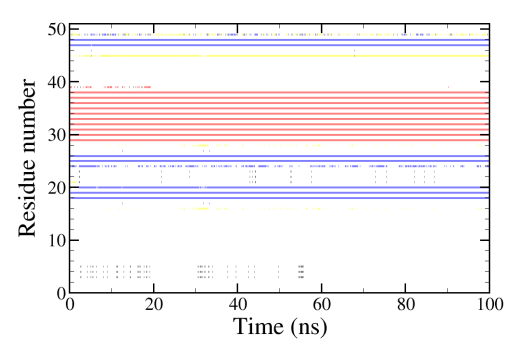

Layer Scheme 2 / Replica 1

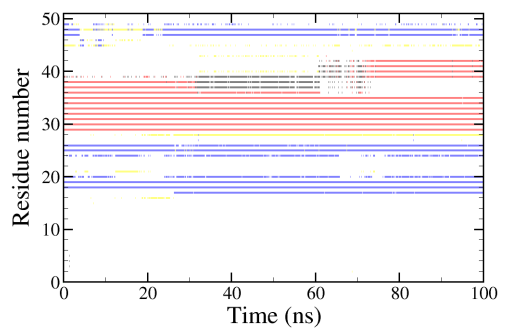

Full CG / Replica 2

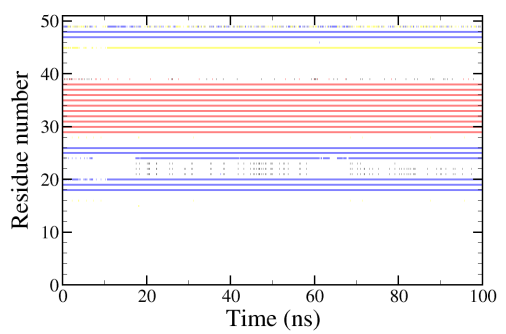

Layer Scheme 2 / Replica 2

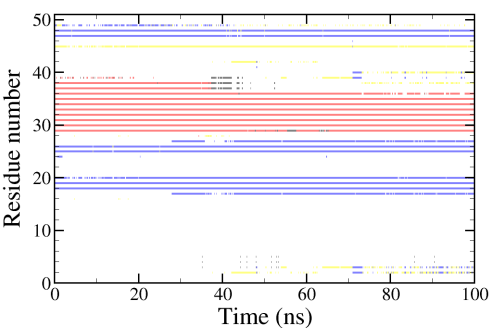

Full AT / Replica 3

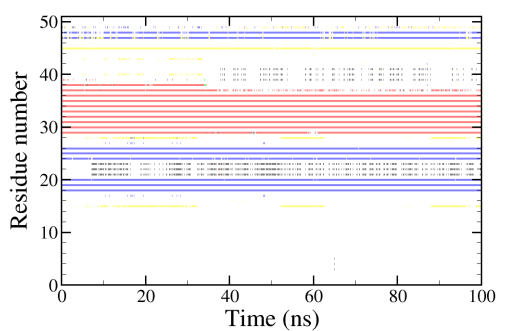

Full CG / Replica 3

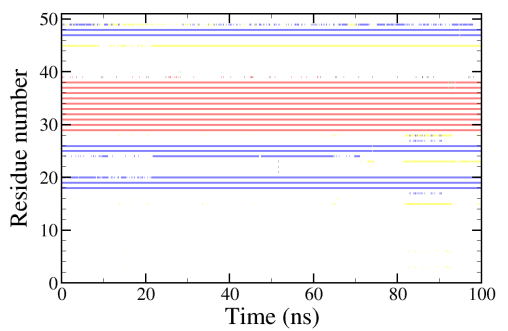

Layer Scheme 2 / Replica 3

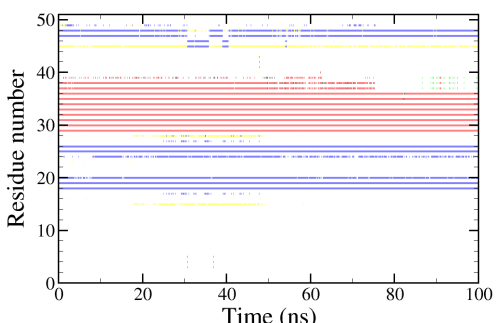

Figure S36: Time series of the secondary structure motifs of the protein in AT water (top), CG water (middle), and AT/CG water with layer scheme 2 (bottom). $\beta$-strands are shown in blue, $\beta$-bridges in yellow, $3_{10}$-helices in black, $\alpha$-helices in red, and $\pi$-helices in green. 


\subsection{Protein 2j8b}

Full AT
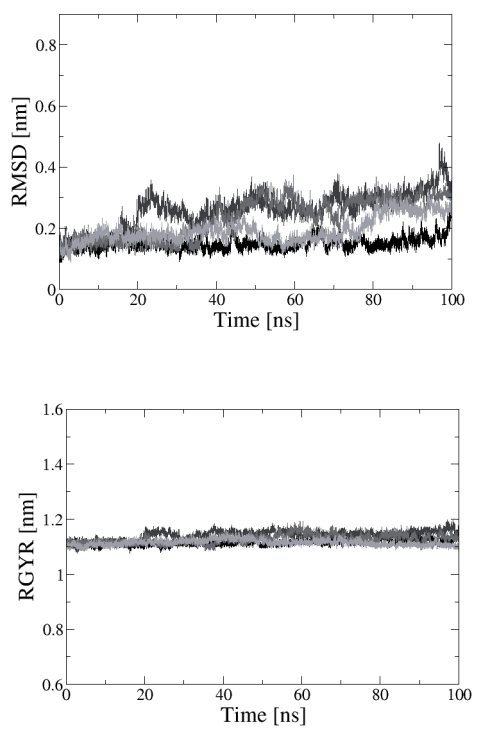

Full CG
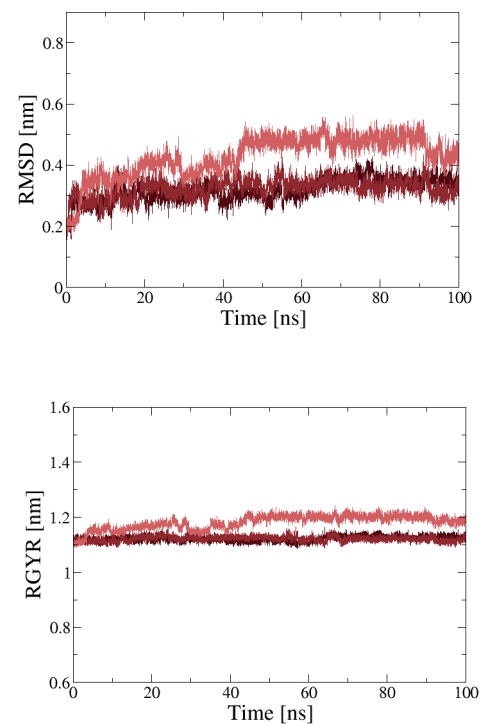

Layer Scheme 2
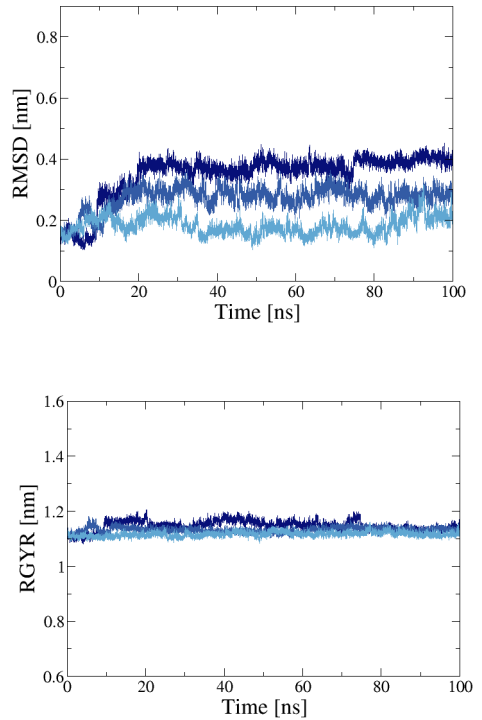

Figure S37: Time series of the backbone atom-positional root-mean-square deviation (RMSD) (top) and the radius of gyration (RGYR) (bottom) of the protein in AT water (black, left), CG water (red, middle), and AT/CG water with layer scheme 2 (blue, right). The replicas are shown in color gradients.
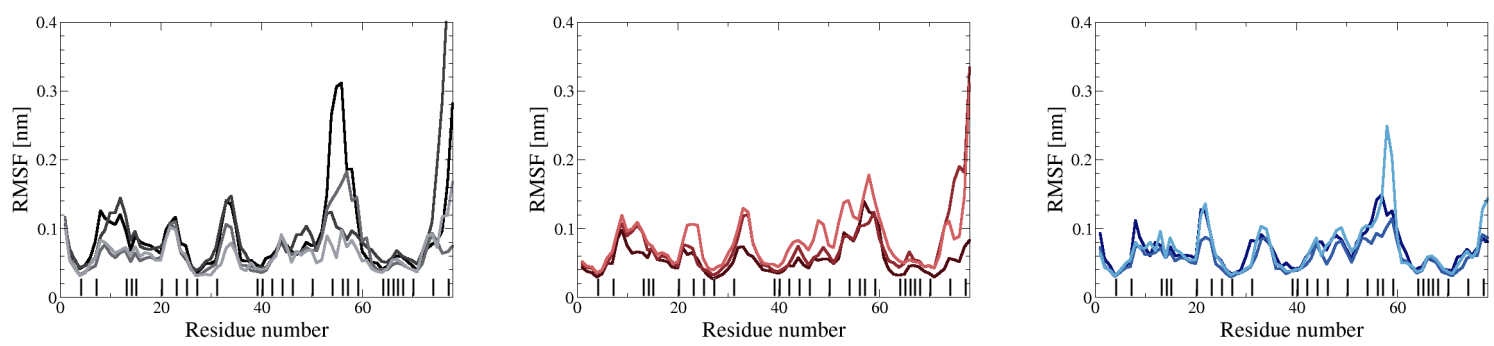

Figure S38: Root-mean-square fluctuation (RMSF) of the $C_{\alpha}$ atoms of the protein in AT water (black, left), CG water (red, middle), and AT/CG water with layer scheme 2 (blue, right). The replicas are shown in color gradients. The positions of the charged residues are indicated with a black dash on the $x$-axis. 
Full AT / Replica 1

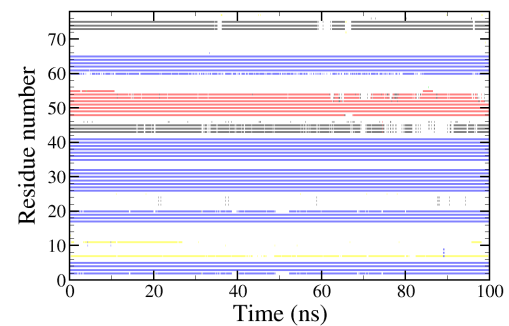

Full AT / Replica 2

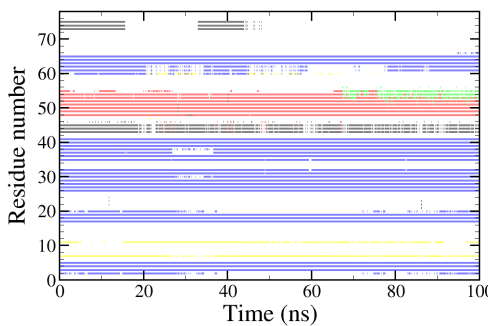

Full AT / Replica 4

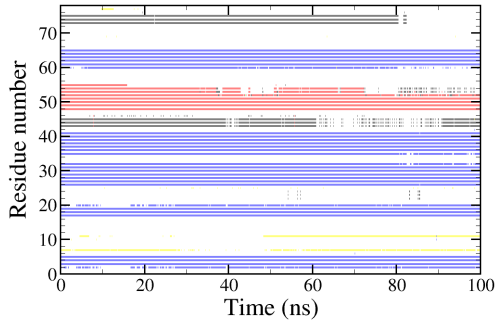

Full CG / Replica 1

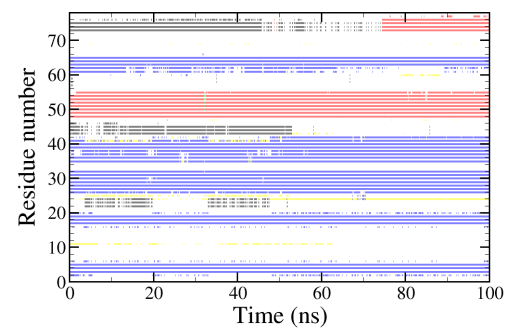

Layer Scheme 2 / Replica 1

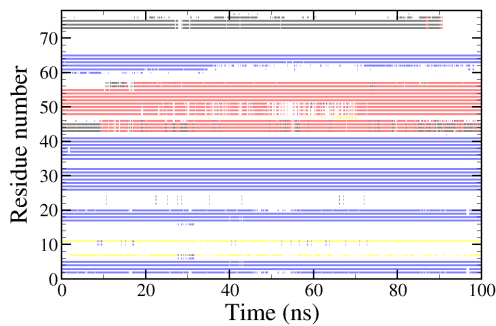

Full CG / Replica 2

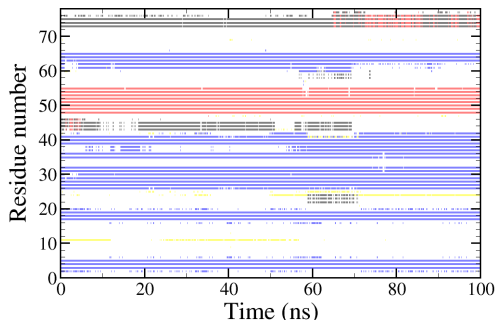

Layer Scheme 2 / Replica 2

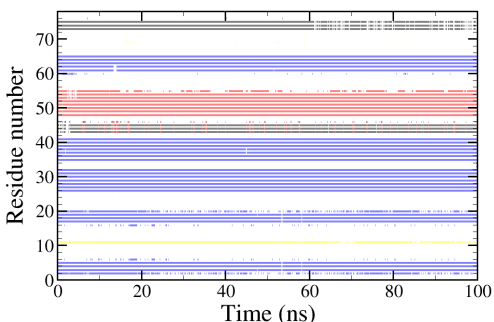

Full AT / Replica 3

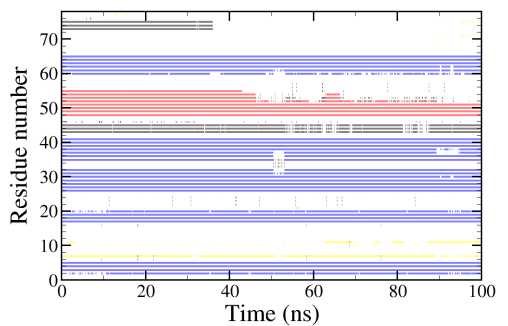

Time (ns)

Figure S39: Time series of the secondary structure motifs of the protein in AT water (top), CG water (middle), and AT/CG water with layer scheme 2 (bottom). $\beta$-strands are shown in blue, $\beta$-bridges in yellow, $3_{10}$-helices in black, $\alpha$-helices in red, and $\pi$-helices in green. 


\subsection{Protein 2nls}

Full AT
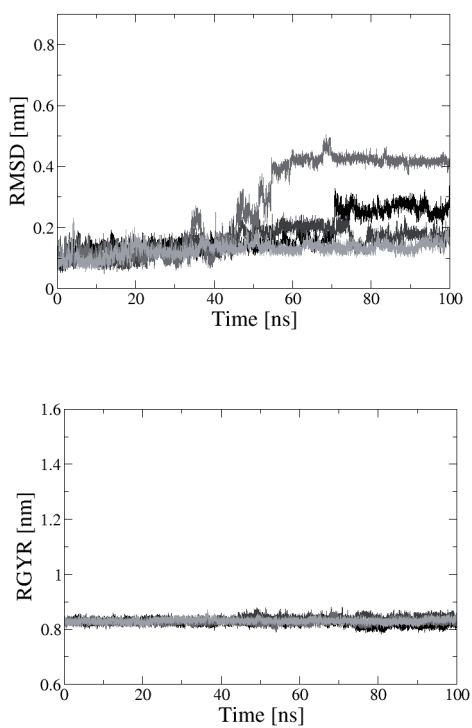

Full CG
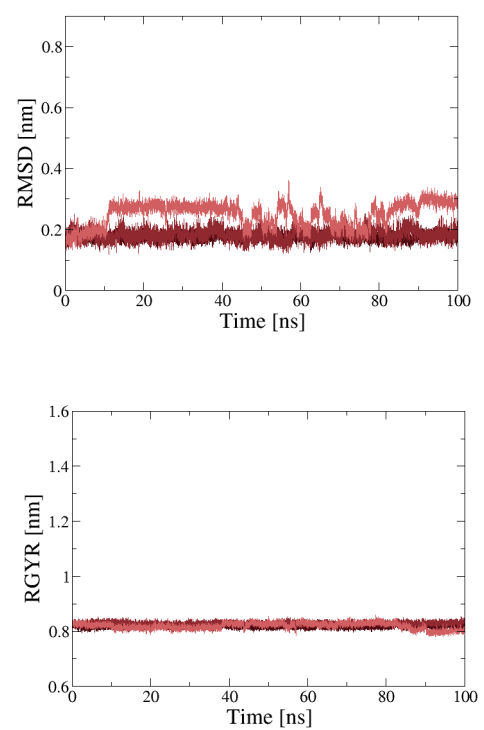

Layer Scheme 2
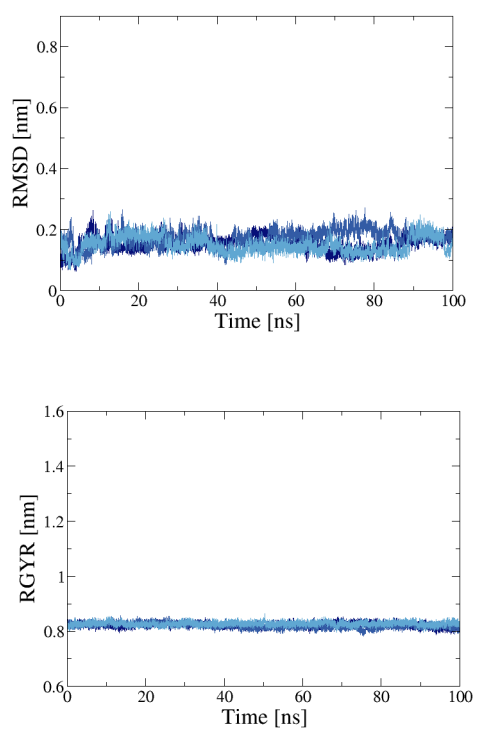

Figure S40: Time series of the backbone atom-positional root-mean-square deviation (RMSD) (top) and the radius of gyration (RGYR) (bottom) of the protein in AT water (black, left), CG water (red, middle), and AT/CG water with layer scheme 2 (blue, right). The replicas are shown in color gradients.
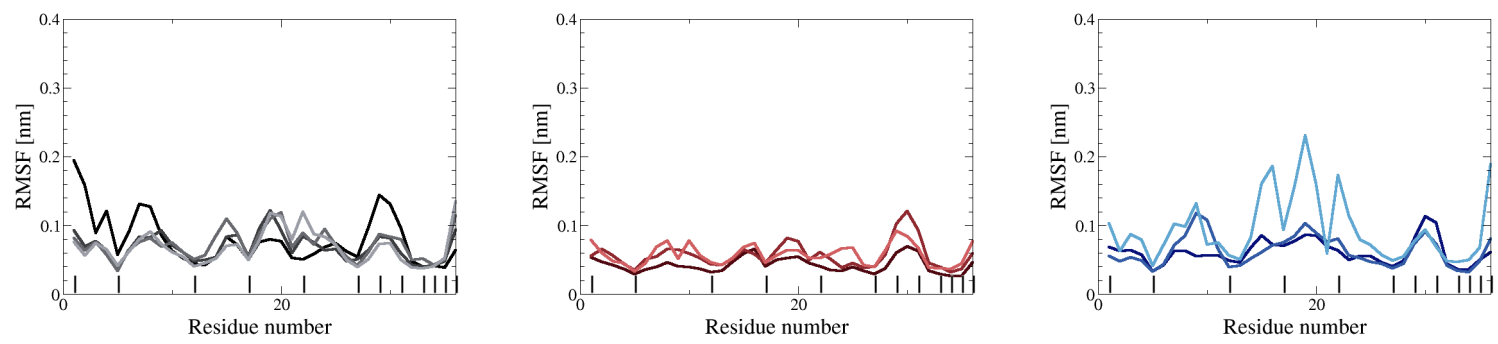

Figure S41: Root-mean-square fluctuation (RMSF) of the $C_{\alpha}$ atoms of the protein in AT water (black, left), CG water (red, middle), and AT/CG water with layer scheme 2 (blue, right). The replicas are shown in color gradients. The positions of the charged residues are indicated with a black dash on the $x$-axis. 
Full AT / Replica 1

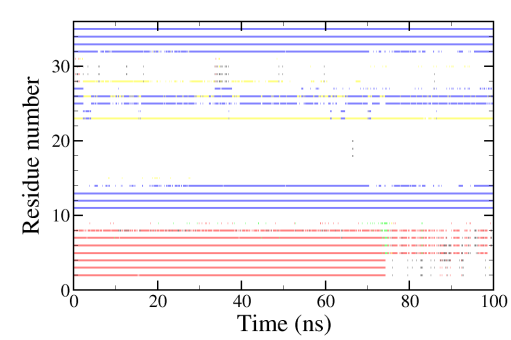

Full AT / Replica 2

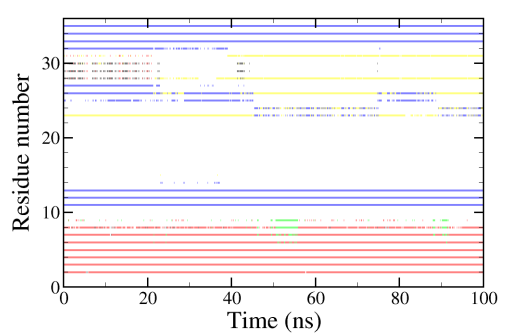

Full AT / Replica 4

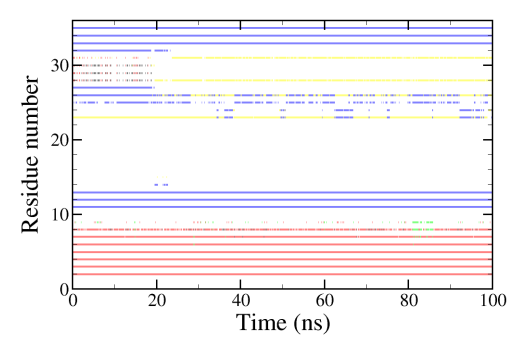

Full CG / Replica 1

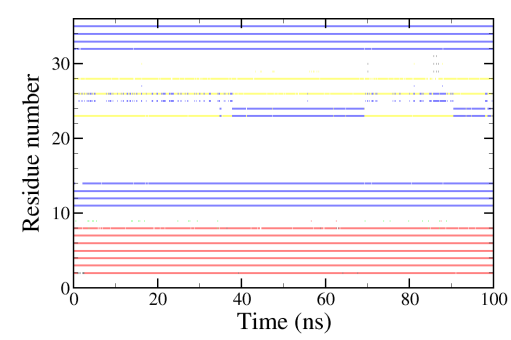

Layer Scheme 2 / Replica 1

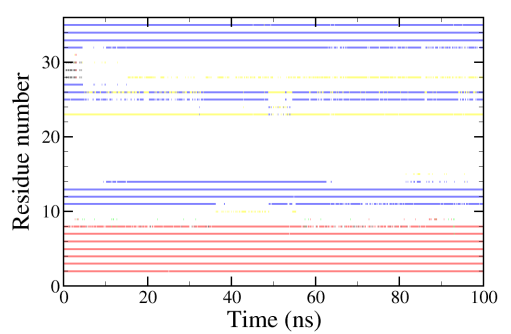

Full CG / Replica 2

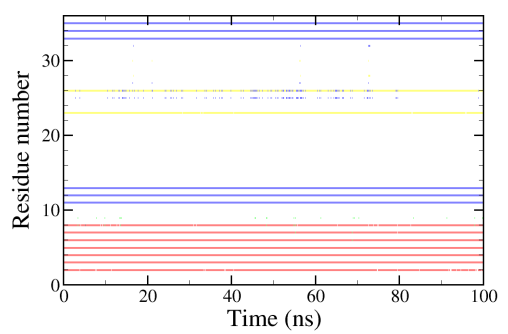

Layer Scheme 2 / Replica 2

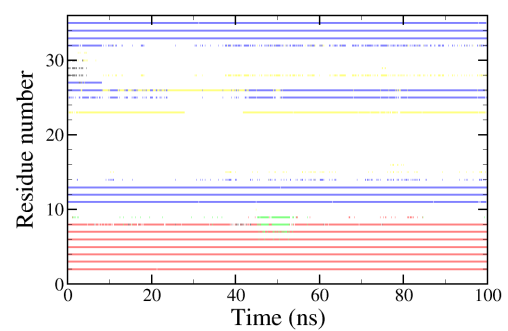

Full AT / Replica 3
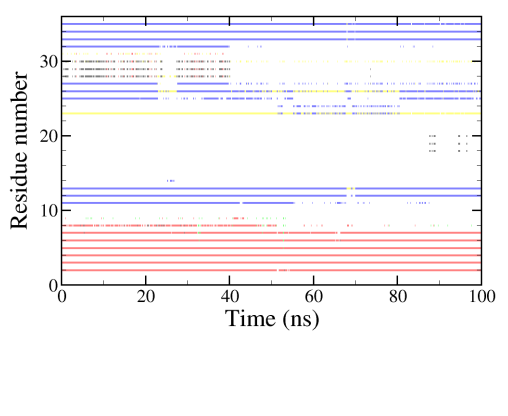


\subsection{Protein 2pnd}

Full AT
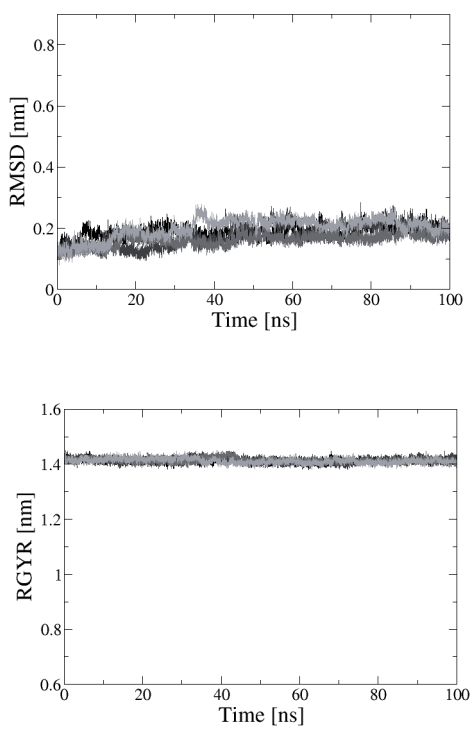

Full CG
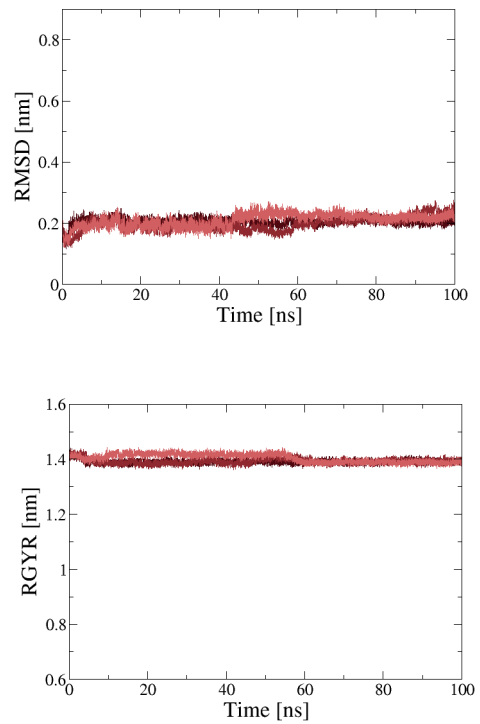

Layer Scheme 2
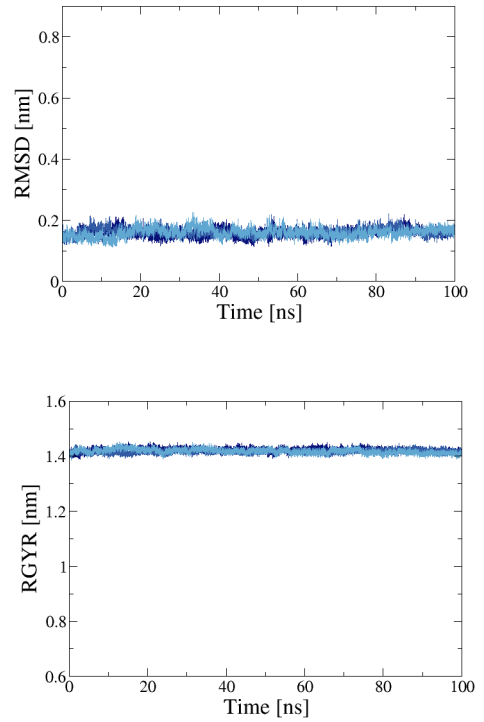

Figure S43: Time series of the backbone atom-positional root-mean-square deviation (RMSD) (top) and the radius of gyration (RGYR) (bottom) of the protein in AT water (black, left), CG water (red, middle), and AT/CG water with layer scheme 2 (blue, right). The replicas are shown in color gradients.
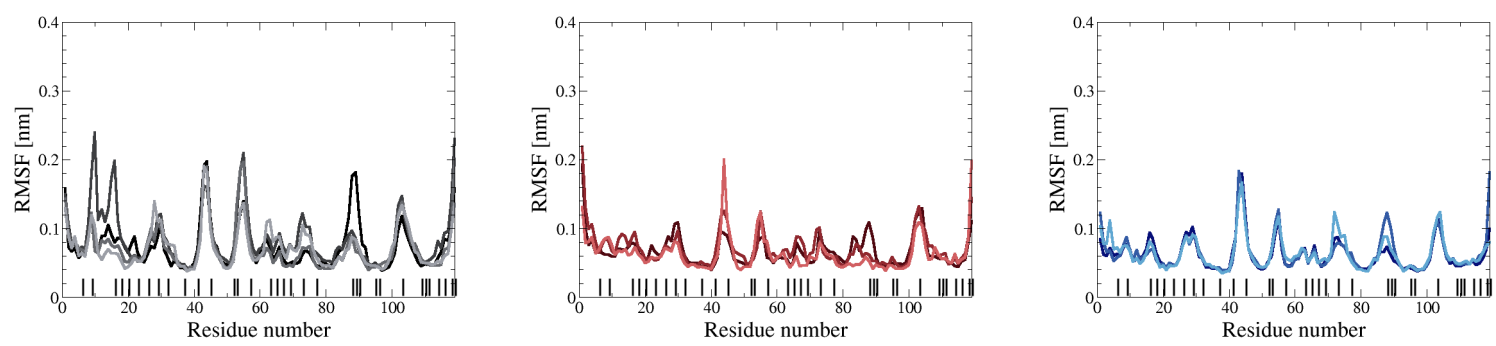

Figure S44: Root-mean-square fluctuation (RMSF) of the $C_{\alpha}$ atoms of the protein in AT water (black, left), CG water (red, middle), and AT/CG water with layer scheme 2 (blue, right). The replicas are shown in color gradients. The positions of the charged residues are indicated with a black dash on the $x$-axis. 
Full AT / Replica 1

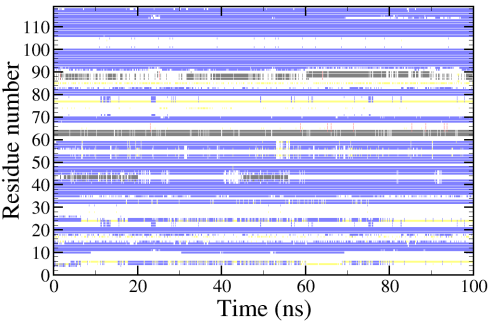

Full AT / Replica 2

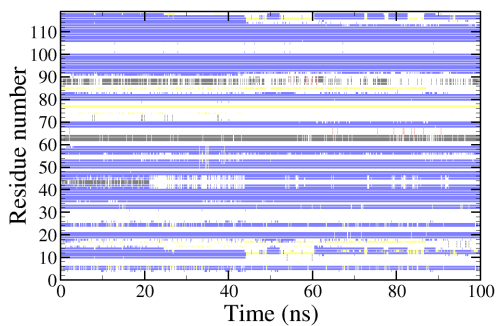

Full AT / Replica 4

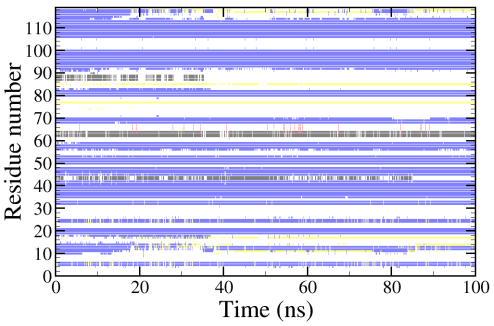

Full CG / Replica 1

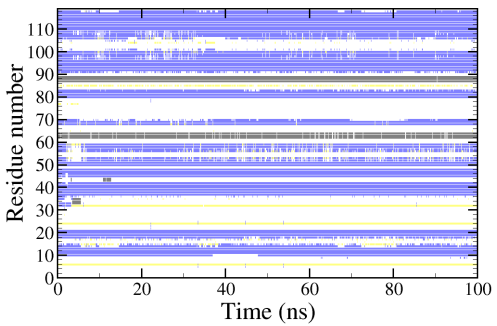

Layer Scheme 2 / Replica 1

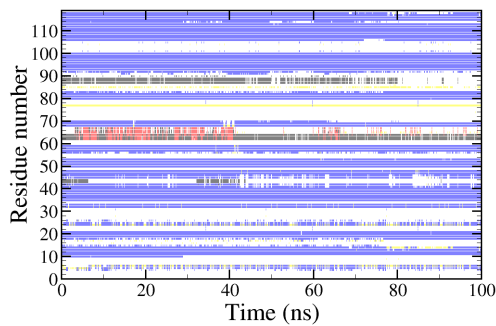

Full CG / Replica 2

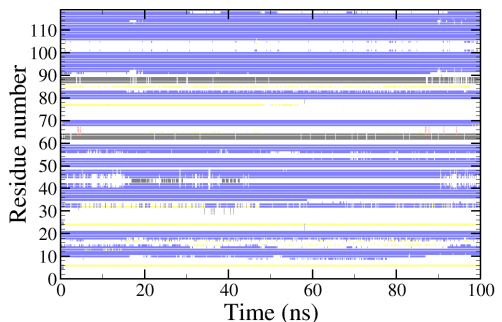

Layer Scheme 2 / Replica 2

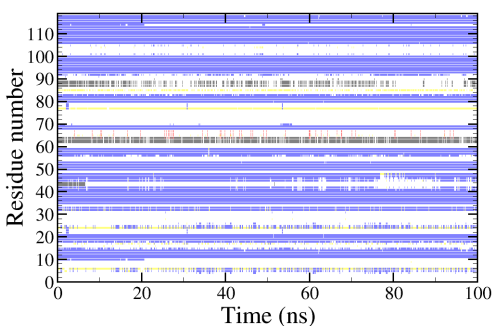

Full AT / Replica 3

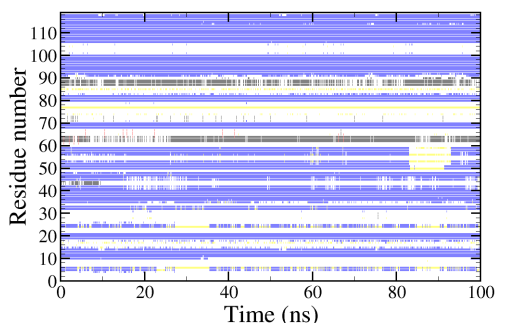

Time (ns)

Figure S45: Time series of the secondary structure motifs of the protein in AT water (top), CG water (middle), and AT/CG water with layer scheme 2 (bottom). $\beta$-strands are shown in blue, $\beta$-bridges in yellow, $3_{10}$-helices in black, $\alpha$-helices in red, and $\pi$-helices in green. 


\subsection{Protein 2pne}

Full AT
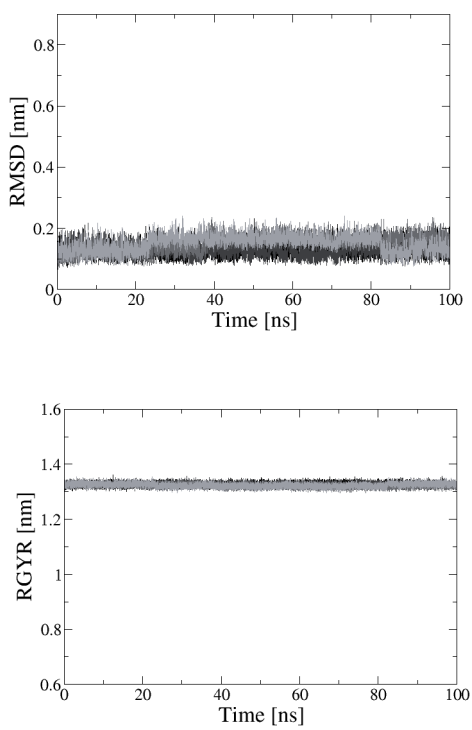

Full CG
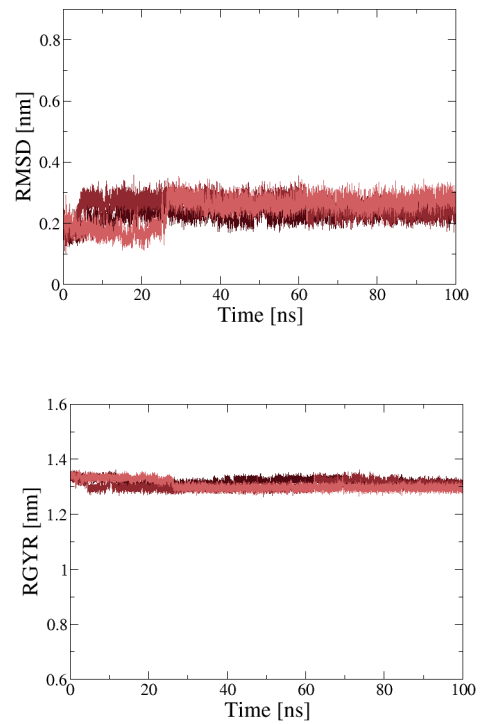

Layer Scheme 2
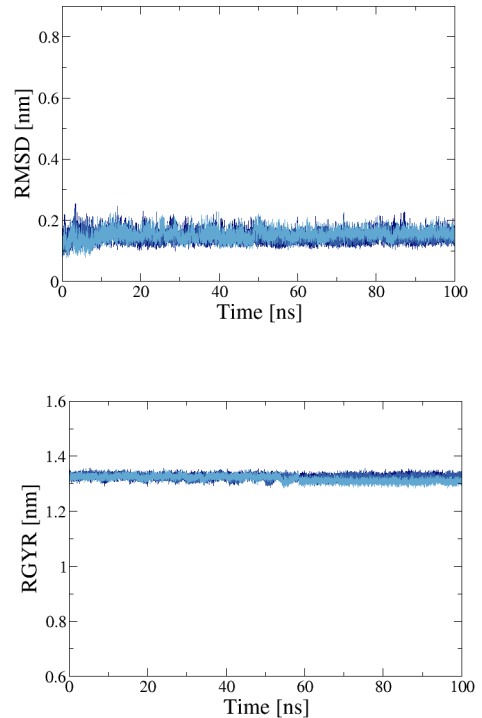

Figure S46: Time series of the backbone atom-positional root-mean-square deviation (RMSD) (top) and the radius of gyration (RGYR) (bottom) of the protein in AT water (black, left), CG water (red, middle), and AT/CG water with layer scheme 2 (blue, right). The replicas are shown in color gradients.
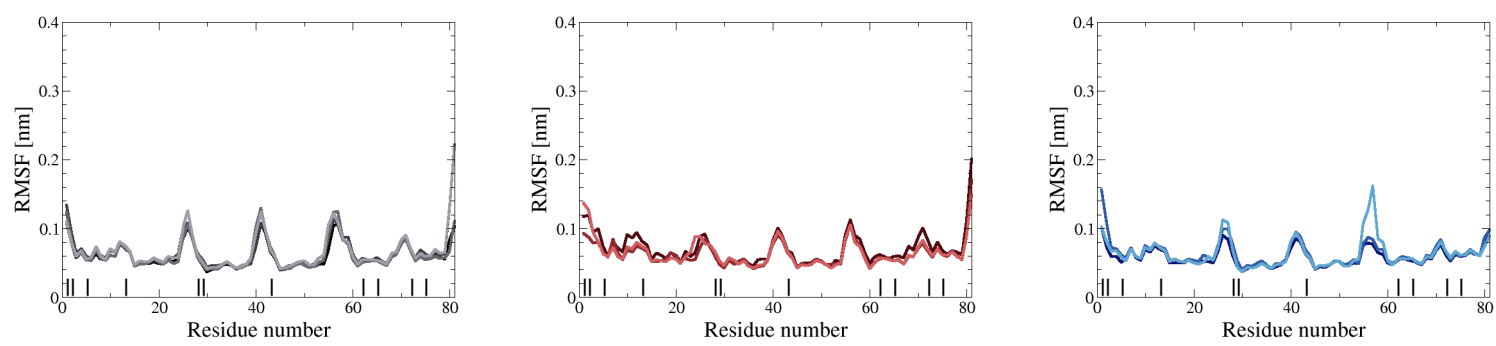

Figure S47: Root-mean-square fluctuation (RMSF) of the $C_{\alpha}$ atoms of the protein in AT water (black, left), CG water (red, middle), and AT/CG water with layer scheme 2 (blue, right). The replicas are shown in color gradients. The positions of the charged residues are indicated with a black dash on the $x$-axis. 

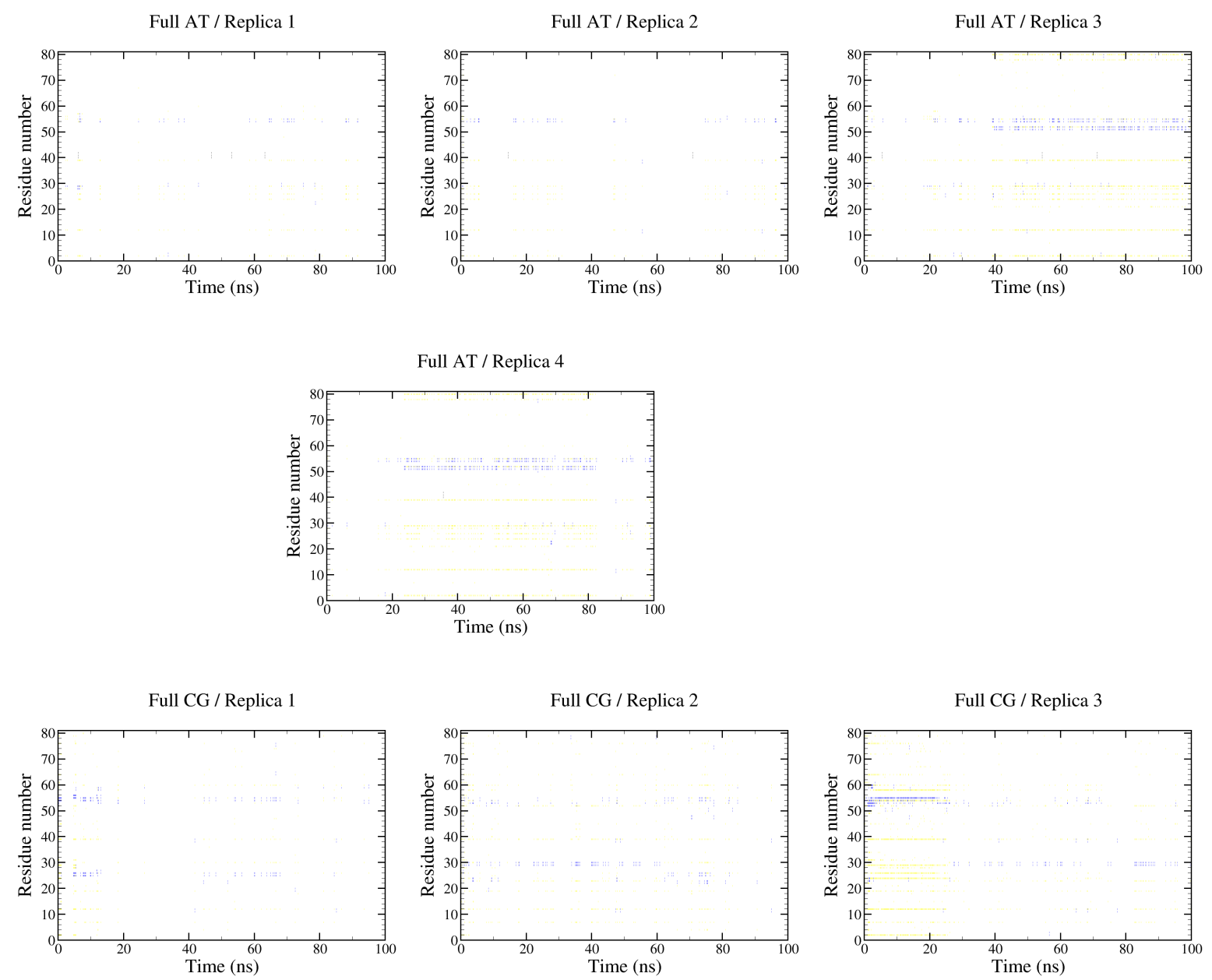

Layer Scheme 2 / Replica 1

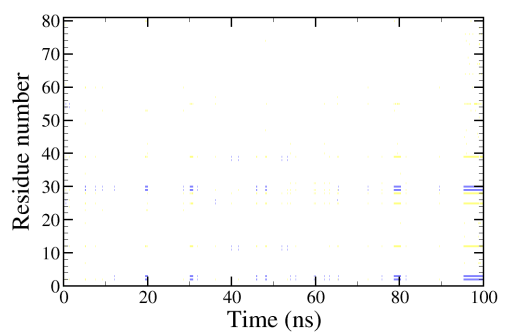

Layer Scheme 2 / Replica 2

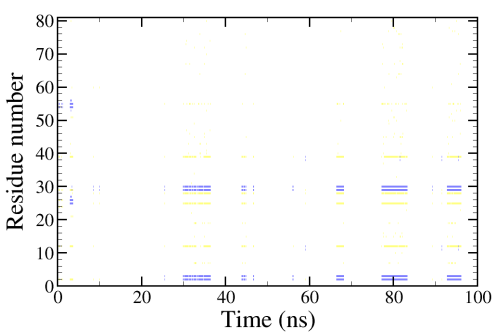

Layer Scheme 2 / Replica 3

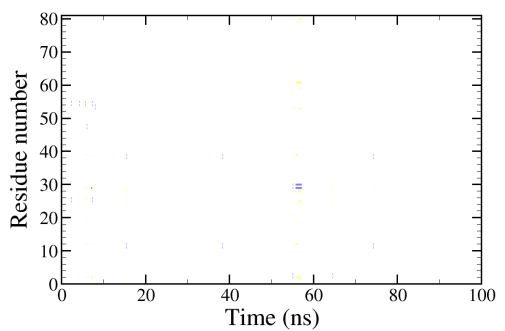

Figure S48: Time series of the secondary structure motifs of the protein in AT water (top), CG water (middle), and AT/CG water with layer scheme 2 (bottom). $\beta$-strands are shown in blue, $\beta$-bridges in yellow, $3_{10}$-helices in black, $\alpha$-helices in red, and $\pi$-helices in green. 


\subsection{Protein 2pth}

Full AT
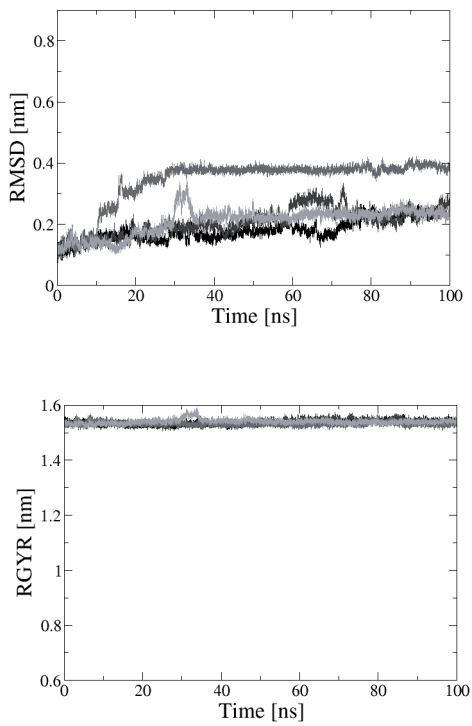

Full CG
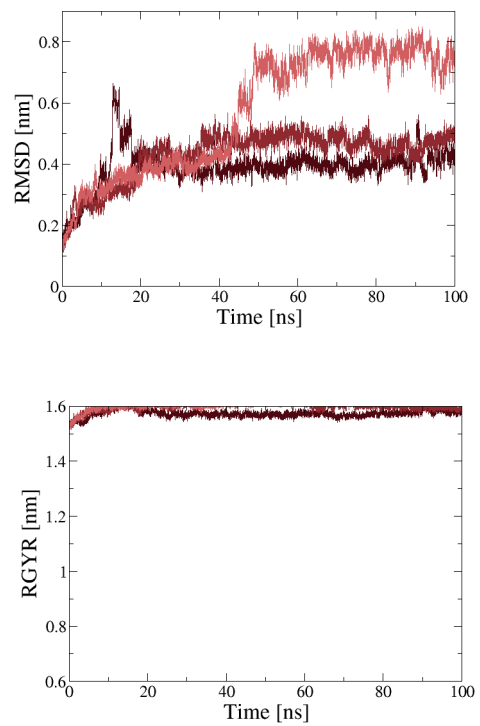

Layer Scheme 2
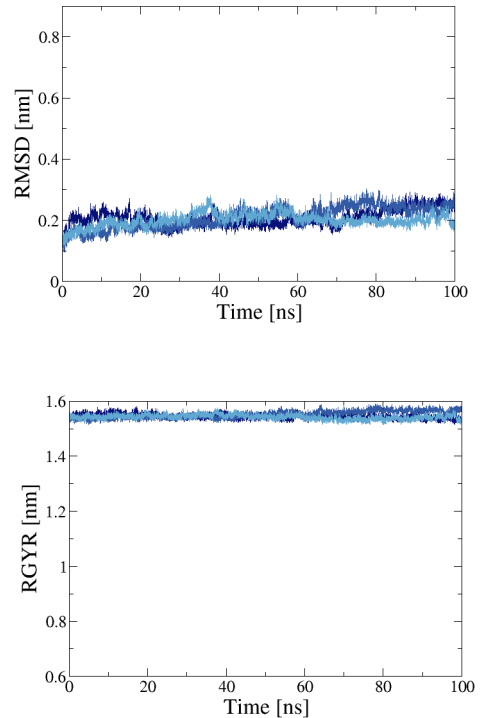

Figure S49: Time series of the backbone atom-positional root-mean-square deviation (RMSD) (top) and the radius of gyration (RGYR) (bottom) of the protein in AT water (black, left), CG water (red, middle), and AT/CG water with layer scheme 2 (blue, right). The replicas are shown in color gradients.
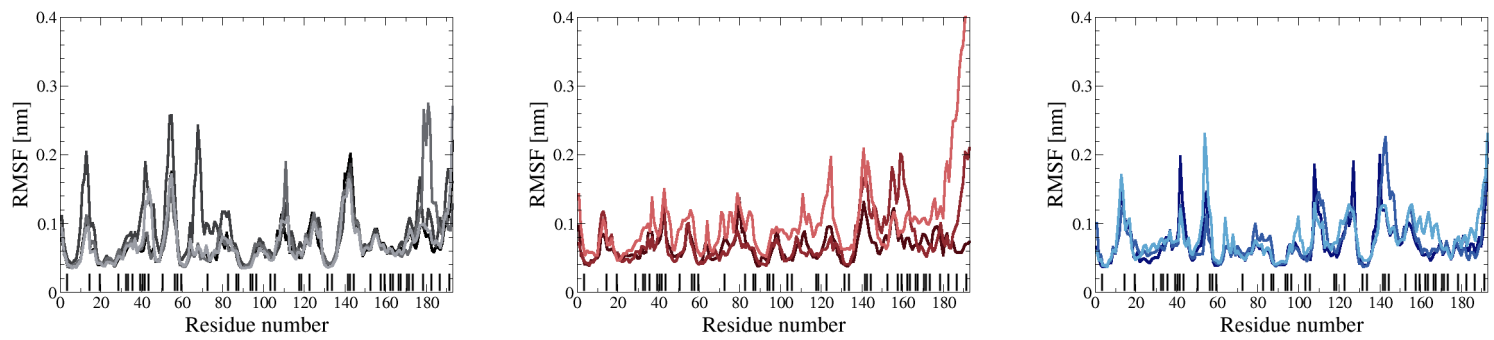

Figure S50: Root-mean-square fluctuation (RMSF) of the $C_{\alpha}$ atoms of the protein in AT water (black, left), CG water (red, middle), and AT/CG water with layer scheme 2 (blue, right). The replicas are shown in color gradients. The positions of the charged residues are indicated with a black dash on the $x$-axis. 
Full AT / Replica 1

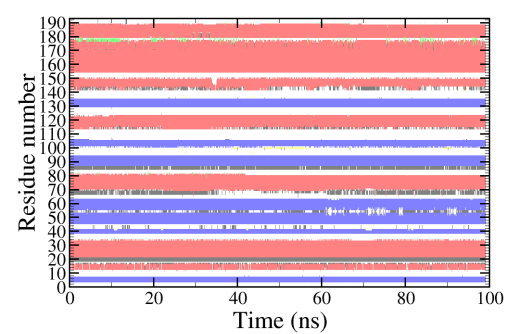

Full AT / Replica 2

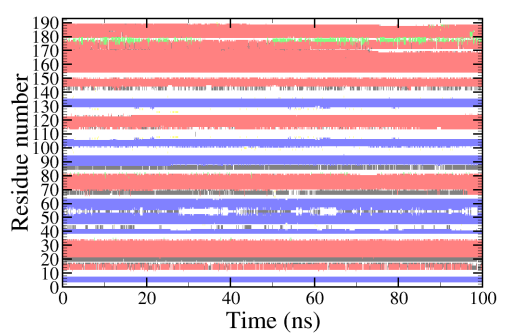

Full AT / Replica 4

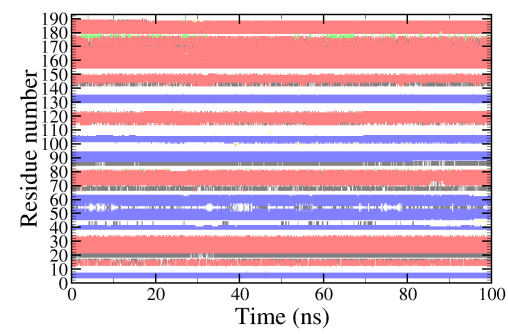

Full CG / Replica 1

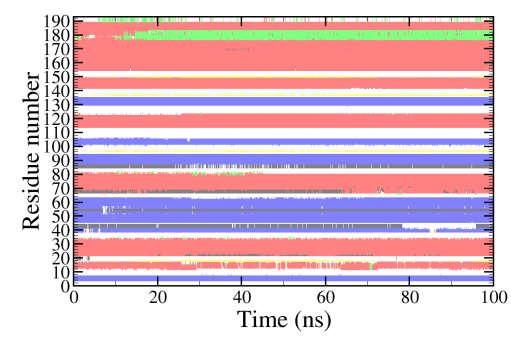

Layer Scheme 2 / Replica 1

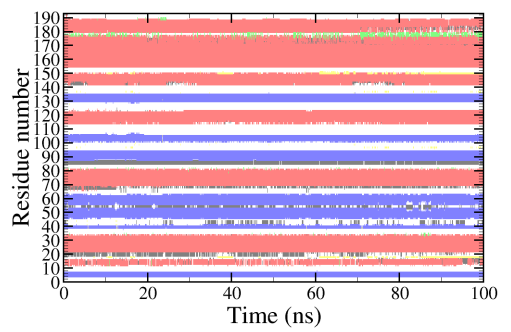

Full CG / Replica 2

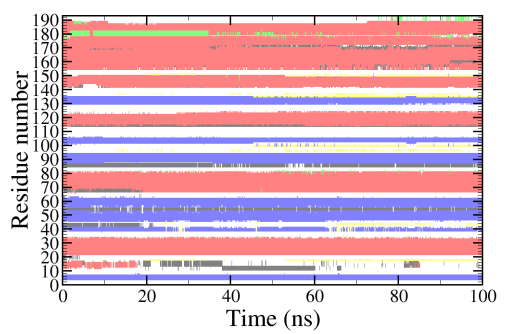

Layer Scheme 2 / Replica 2

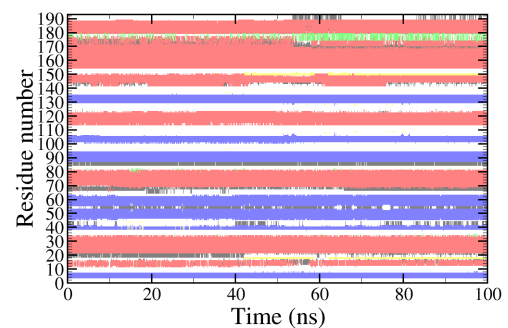

Full AT / Replica 3

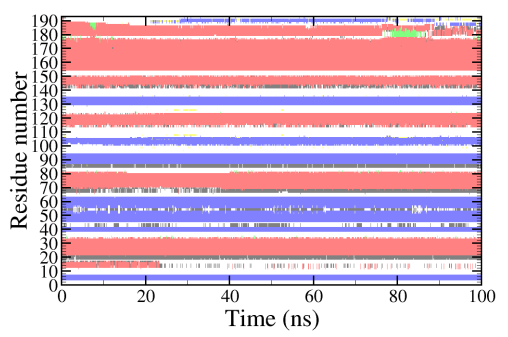




\subsection{Protein 2wlw}

Full AT
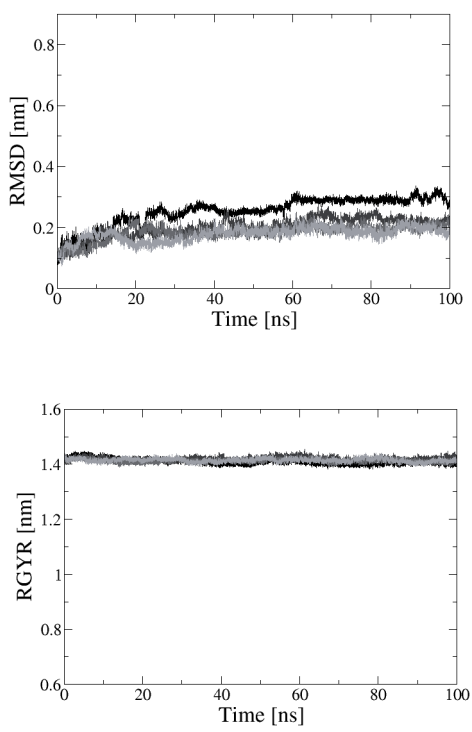

Full CG
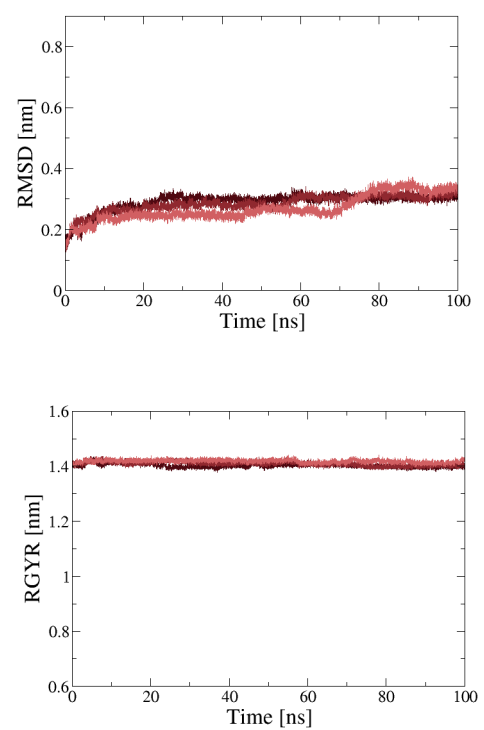

Layer Scheme 2
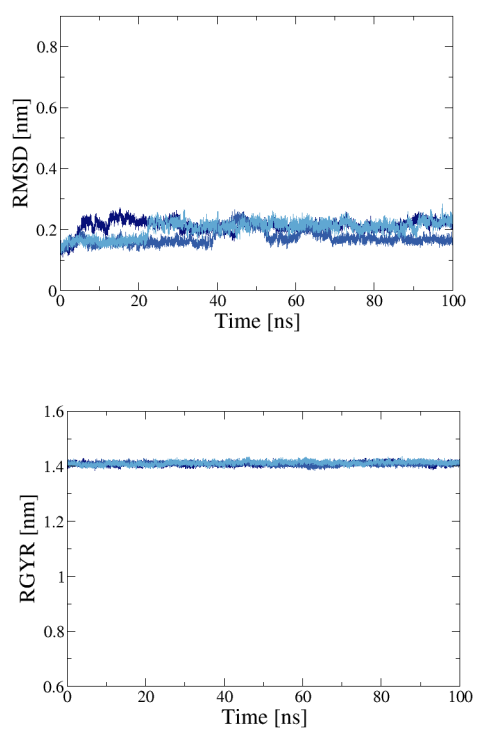

Figure S52: Time series of the backbone atom-positional root-mean-square deviation (RMSD) (top) and the radius of gyration (RGYR) (bottom) of the protein in AT water (black, left), CG water (red, middle), and AT/CG water with layer scheme 2 (blue, right). The replicas are shown in color gradients.
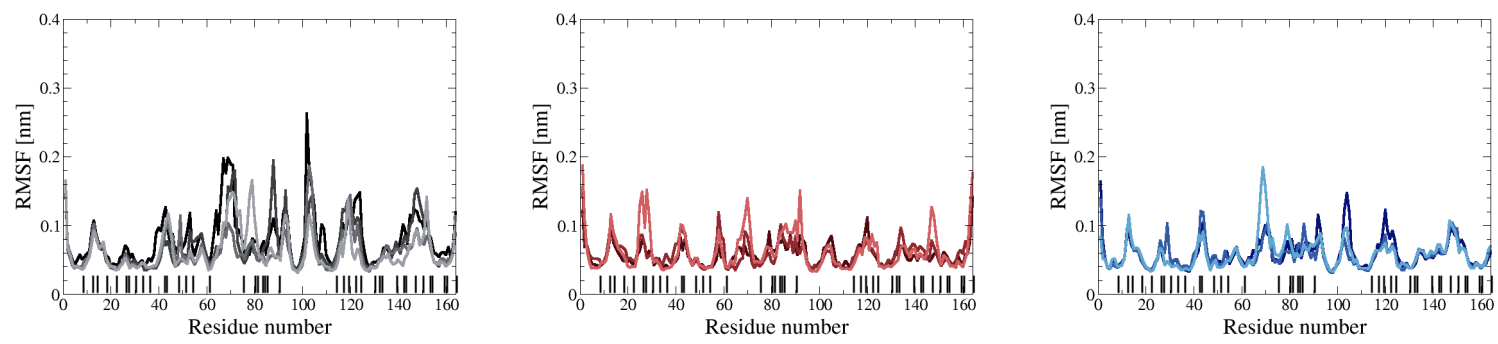

Figure S53: Root-mean-square fluctuation (RMSF) of the $C_{\alpha}$ atoms of the protein in AT water (black, left), CG water (red, middle), and AT/CG water with layer scheme 2 (blue, right). The replicas are shown in color gradients. The positions of the charged residues are indicated with a black dash on the $x$-axis. 
Full AT / Replica 1

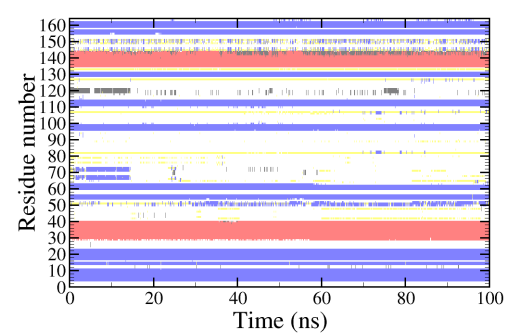

Full AT / Replica 2

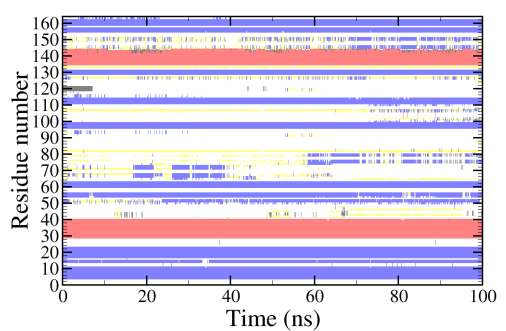

Full AT / Replica 4

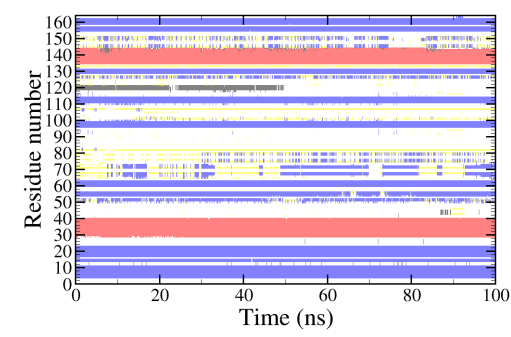

Full CG / Replica 1

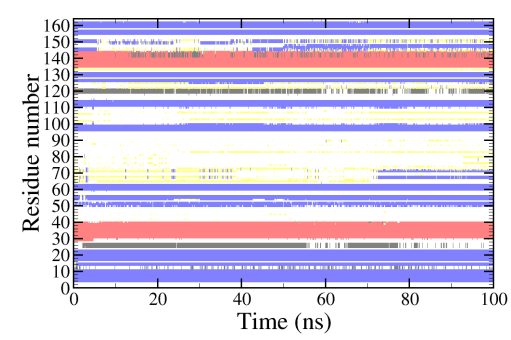

Layer Scheme 2 / Replica 1

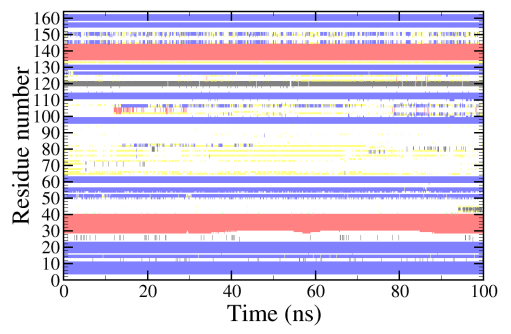

Full CG / Replica 2

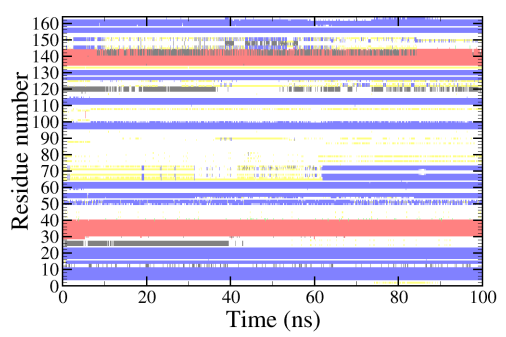

Layer Scheme 2 / Replica 2

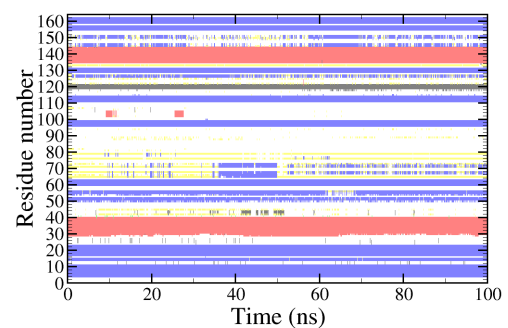

Full AT / Replica 3

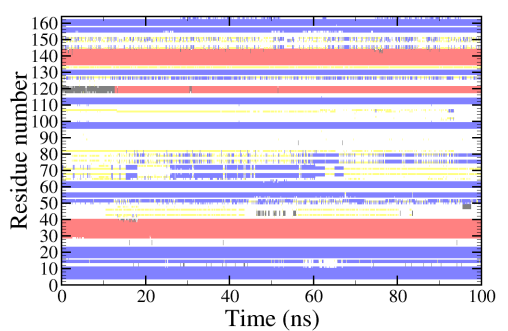

Time (ns)

Figure S54: Time series of the secondary structure motifs of the protein in AT water (top), CG water (middle), and AT/CG water with layer scheme 2 (bottom). $\beta$-strands are shown in blue, $\beta$-bridges in yellow, $3_{10}$-helices in black, $\alpha$-helices in red, and $\pi$-helices in green. 


\subsection{Protein 2yxf}

Full AT
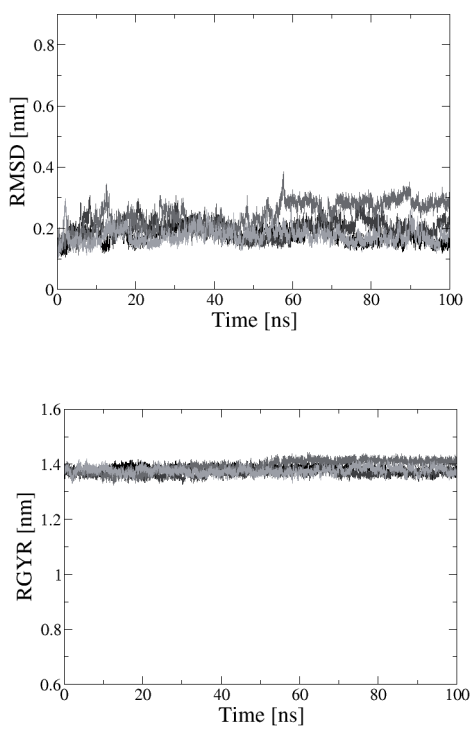

Full CG
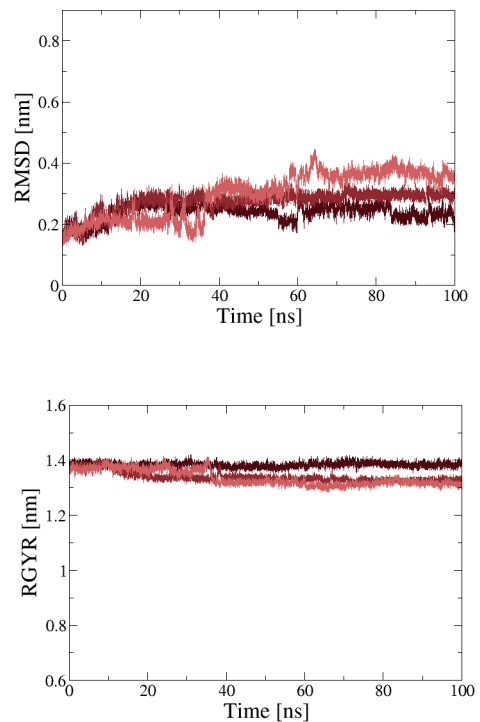

Layer Scheme 2
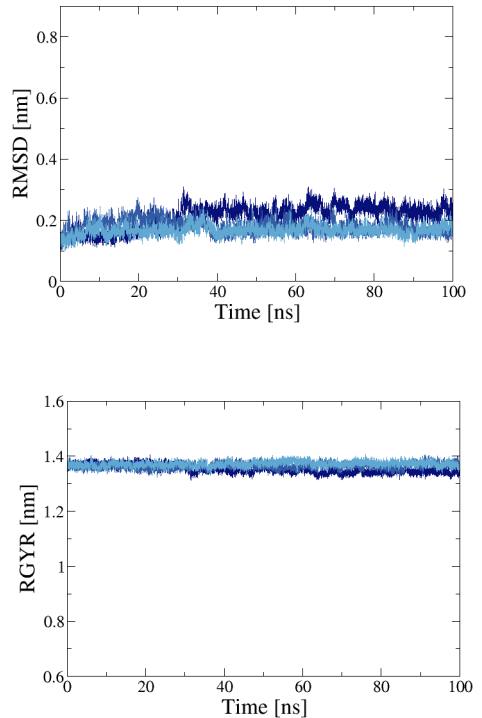

Figure S55: Time series of the backbone atom-positional root-mean-square deviation (RMSD) (top) and the radius of gyration (RGYR) (bottom) of the protein in AT water (black, left), CG water (red, middle), and AT/CG water with layer scheme 2 (blue, right). The replicas are shown in color gradients.
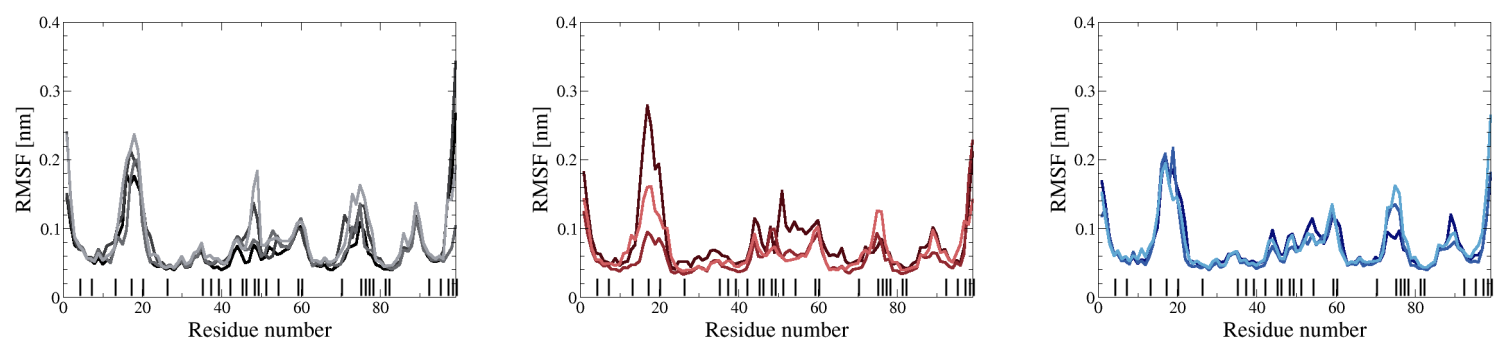

Figure S56: Root-mean-square fluctuation (RMSF) of the $C_{\alpha}$ atoms of the protein in AT water (black, left), CG water (red, middle), and AT/CG water with layer scheme 2 (blue, right). The replicas are shown in color gradients. The positions of the charged residues are indicated with a black dash on the $x$-axis. 
Full AT / Replica 1

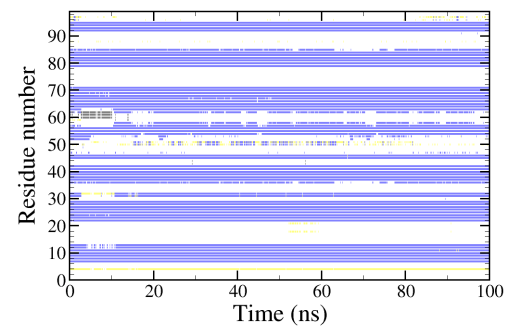

Full AT / Replica 2

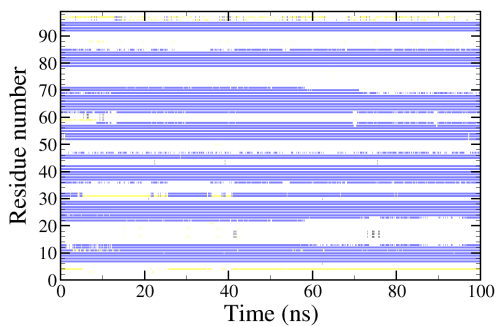

Full AT / Replica 4

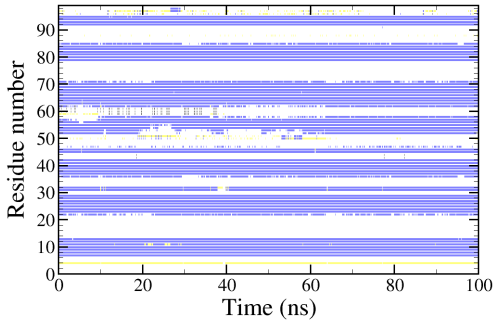

Full CG / Replica 1

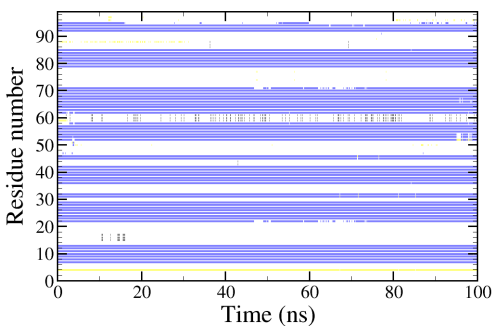

Layer Scheme 2 / Replica 1

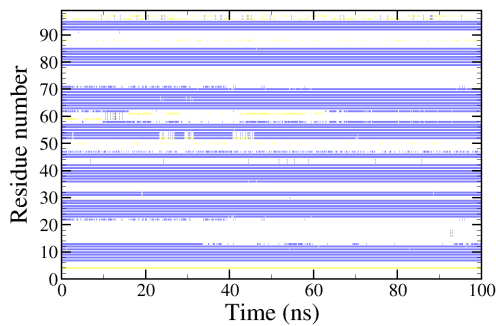

Full CG / Replica 2

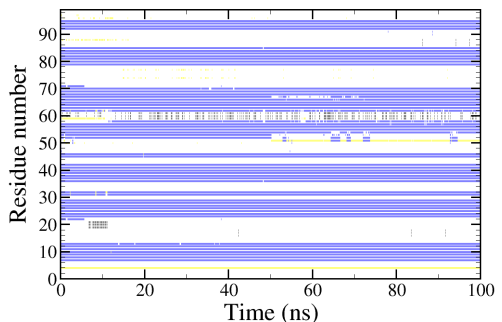

Layer Scheme 2 / Replica 2

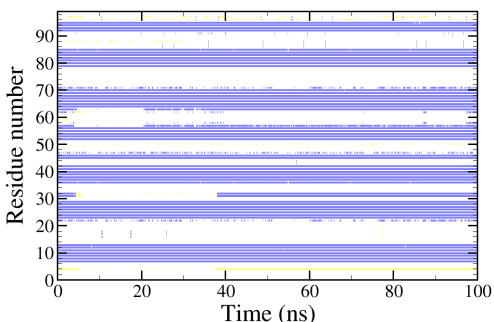

Full AT / Replica 3

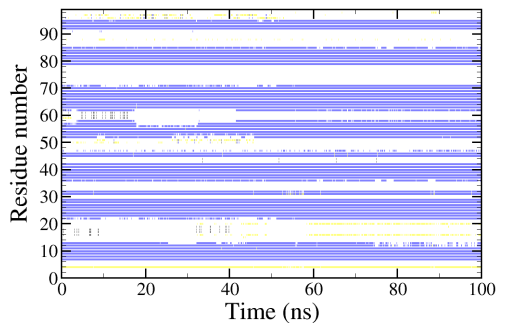

Full CG / Replica 3

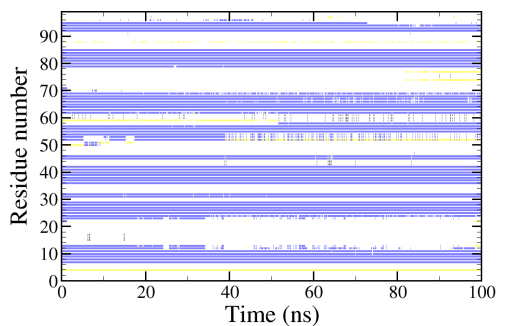

Layer Scheme 2 / Replica 3

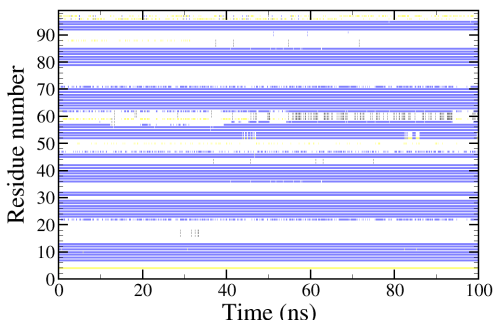

Figure S57: Time series of the secondary structure motifs of the protein in AT water (top), CG water (middle), and AT/CG water with layer scheme 2 (bottom). $\beta$-strands are shown in blue, $\beta$-bridges in yellow, $3_{10}$-helices in black, $\alpha$-helices in red, and $\pi$-helices in green. 


\subsection{Protein $3 \mathrm{ca} 7$}

Full AT
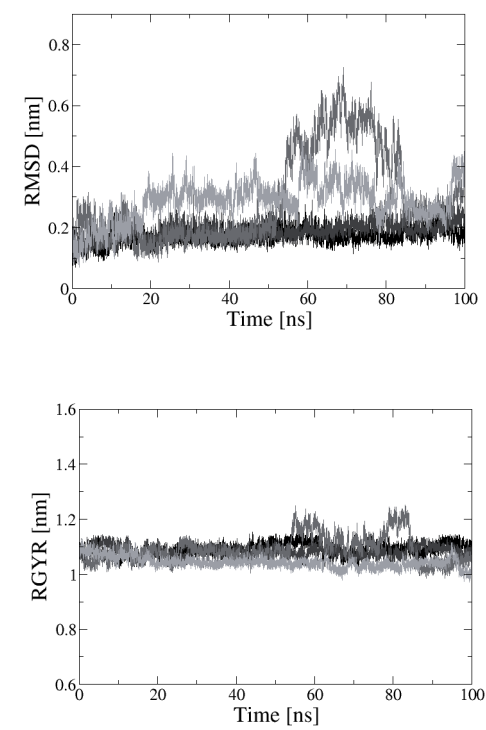

Full CG
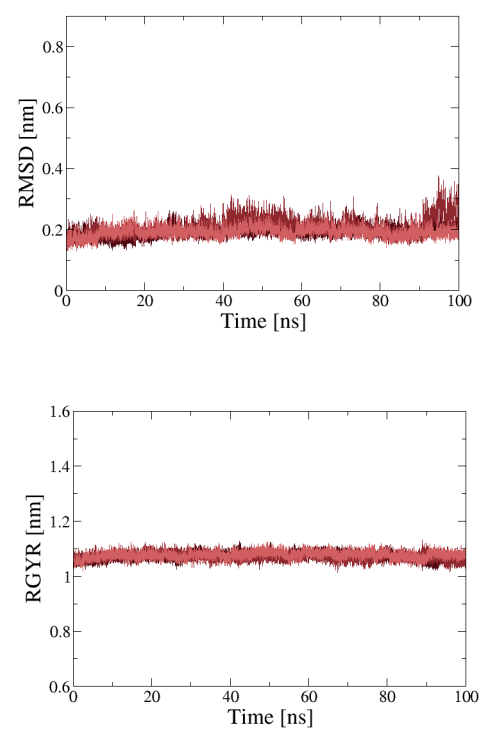

Layer Scheme 2
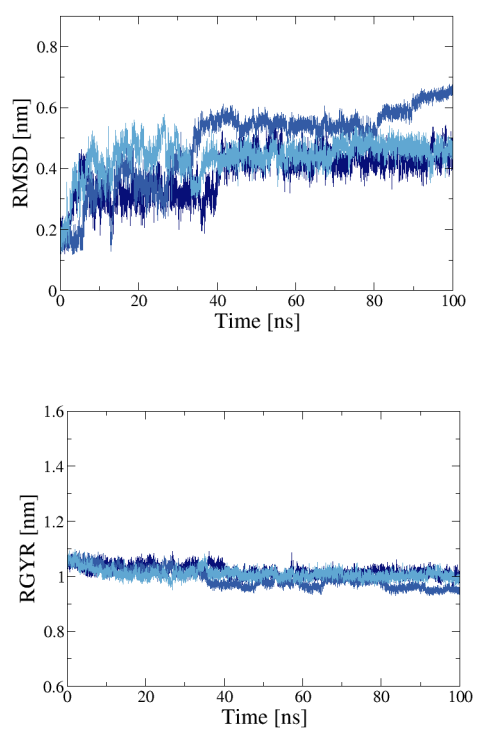

Figure S58: Time series of the backbone atom-positional root-mean-square deviation (RMSD) (top) and the radius of gyration (RGYR) (bottom) of the protein in AT water (black, left), CG water (red, middle), and AT/CG water with layer scheme 2 (blue, right). The replicas are shown in color gradients.
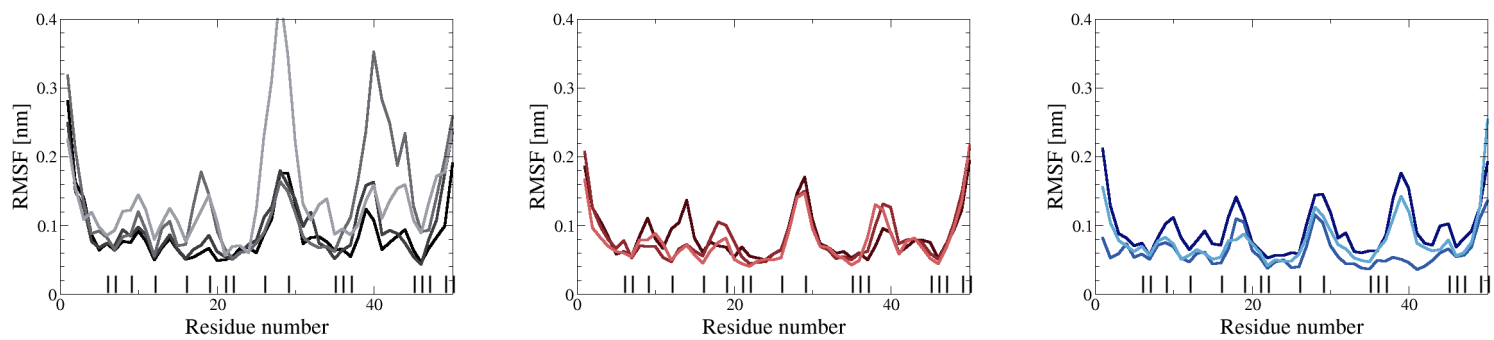

Figure S59: Root-mean-square fluctuation (RMSF) of the $C_{\alpha}$ atoms of the protein in AT water (black, left), CG water (red, middle), and AT/CG water with layer scheme 2 (blue, right). The replicas are shown in color gradients. The positions of the charged residues are indicated with a black dash on the $x$-axis. 
Full AT / Replica 1

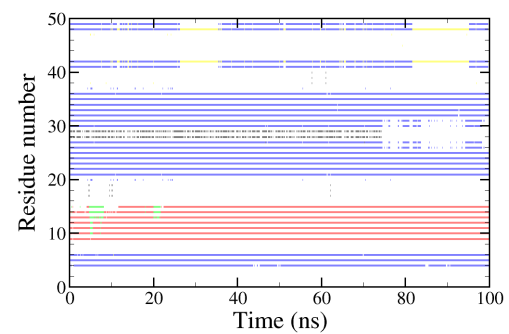

Full AT / Replica 2

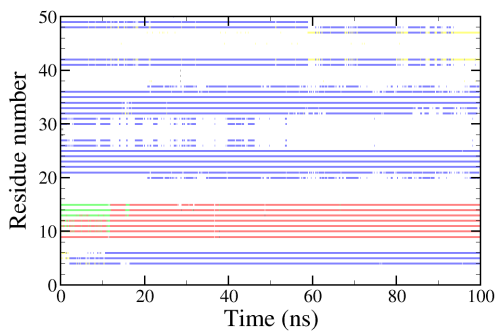

Full AT / Replica 4

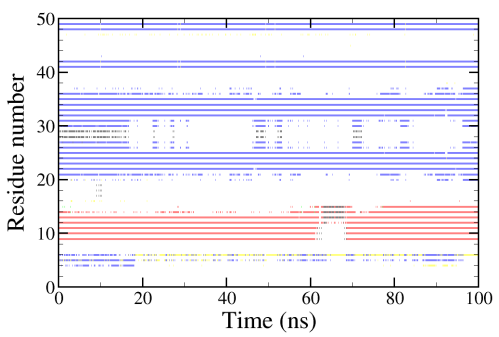

Full CG / Replica 1

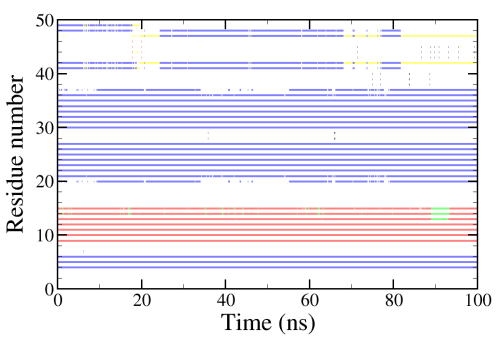

Layer Scheme 2 / Replica 1

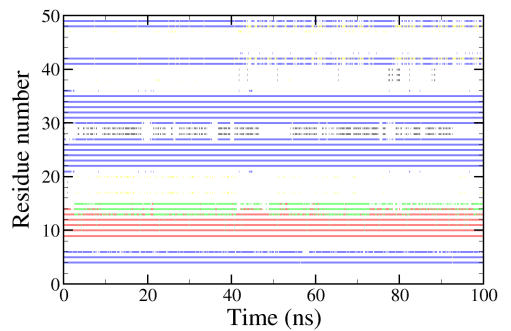

Full CG / Replica 2

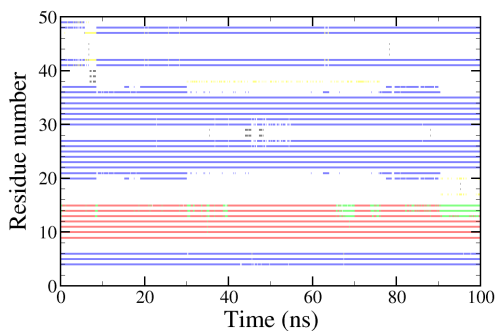

Layer Scheme 2 / Replica 2

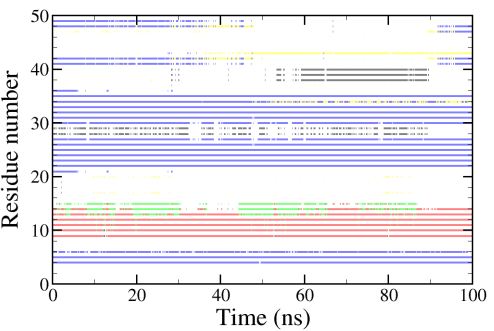

Full AT / Replica 3

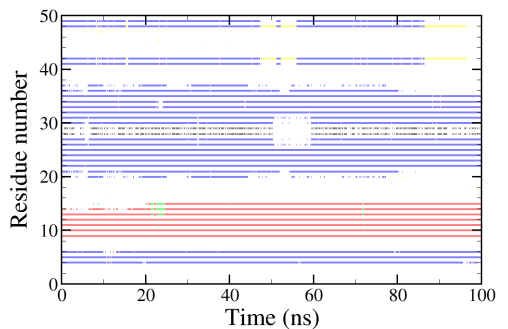




\subsection{Protein 3 ci2}

Full AT
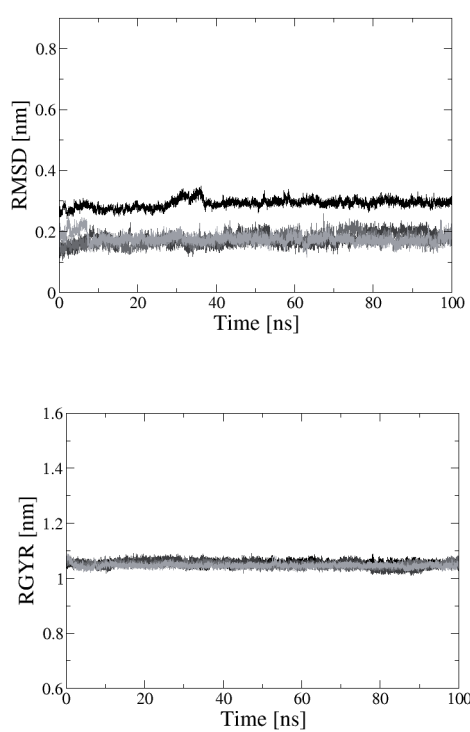

Full CG
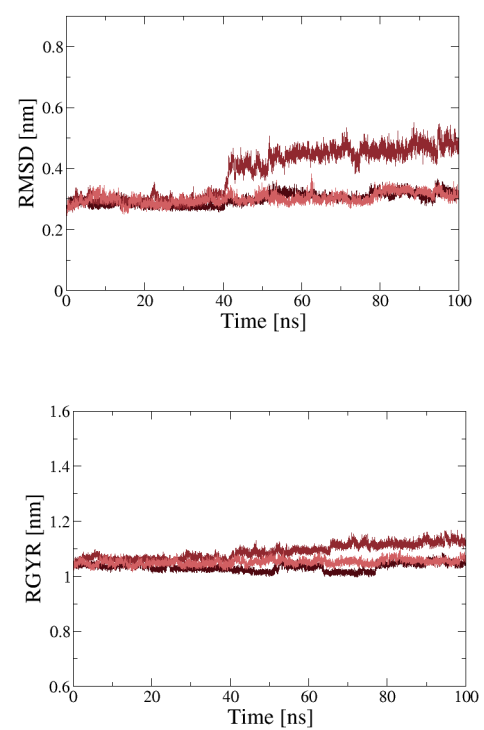

Layer Scheme 2
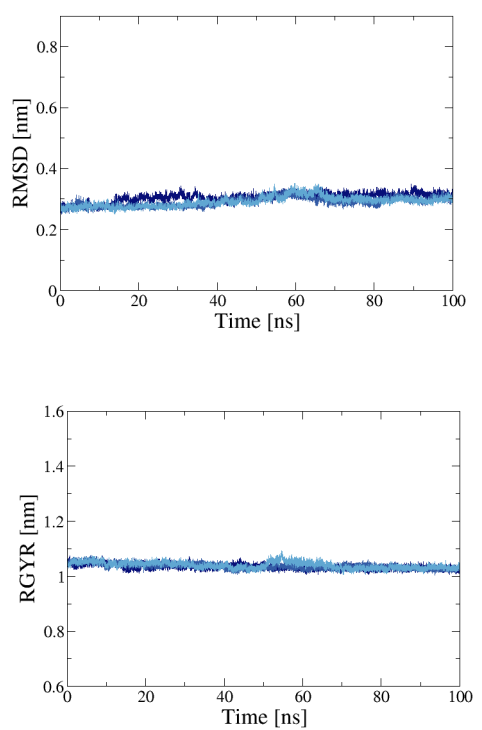

Figure S61: Time series of the backbone atom-positional root-mean-square deviation (RMSD) (top) and the radius of gyration (RGYR) (bottom) of the protein in AT water (black, left), CG water (red, middle), and AT/CG water with layer scheme 2 (blue, right). The replicas are shown in color gradients.
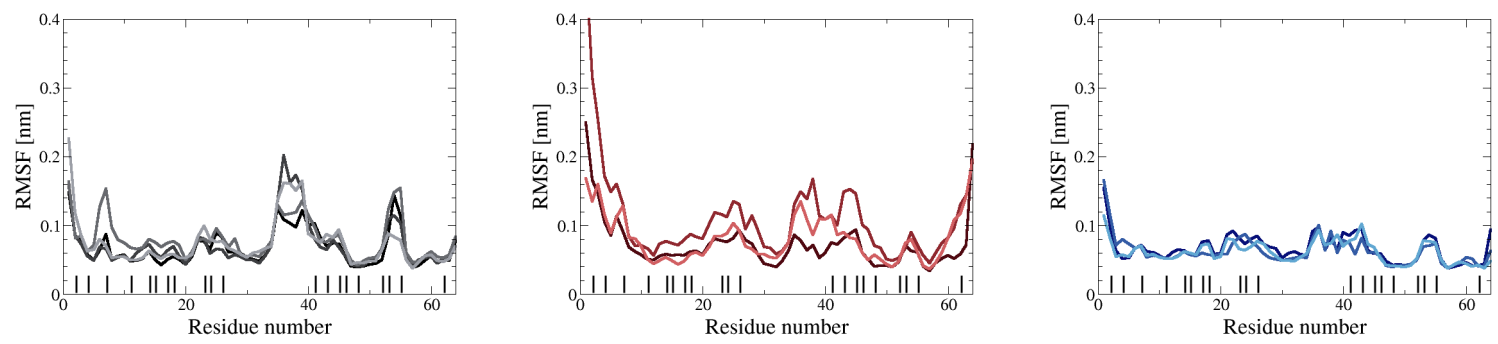

Figure S62: Root-mean-square fluctuation (RMSF) of the $C_{\alpha}$ atoms of the protein in AT water (black, left), CG water (red, middle), and AT/CG water with layer scheme 2 (blue, right). The replicas are shown in color gradients. The positions of the charged residues are indicated with a black dash on the $x$-axis. 
Full AT / Replica 1

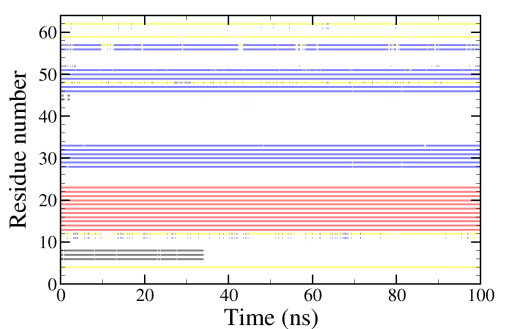

Full AT / Replica 2

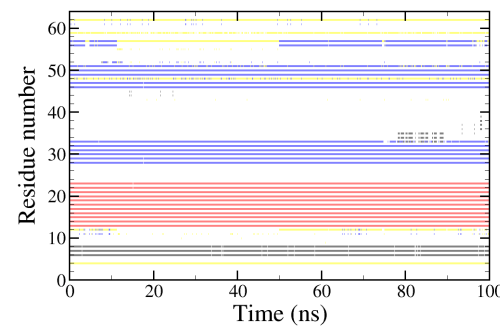

Full AT / Replica 4

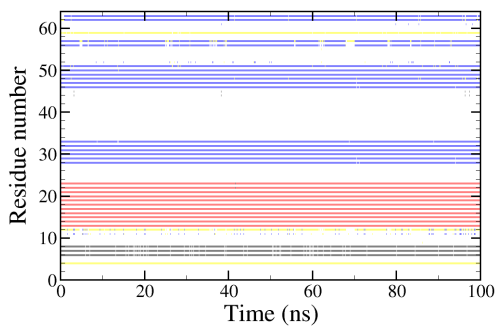

Full CG / Replica 1

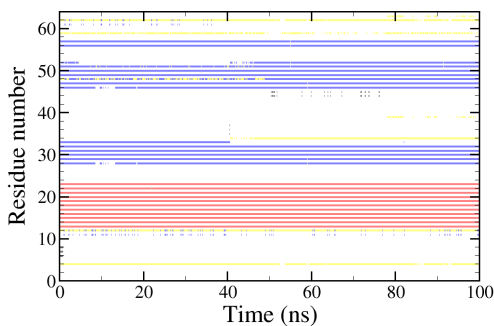

Layer Scheme 2 / Replica 1

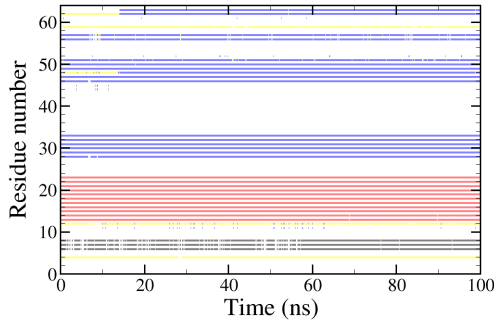

Full CG / Replica 2

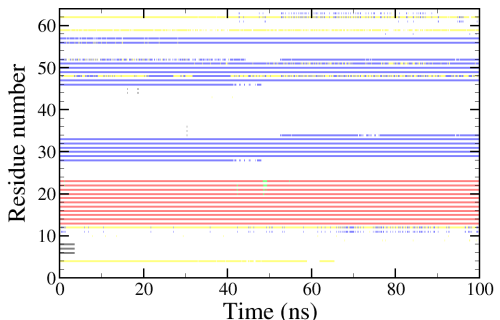

Layer Scheme 2 / Replica 2

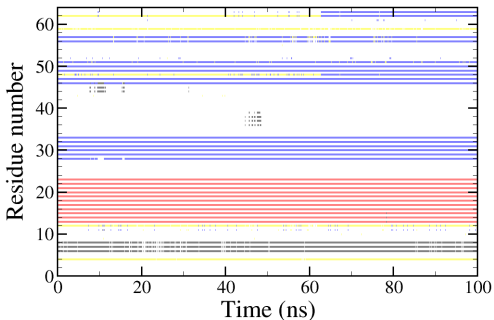

Full AT / Replica 3

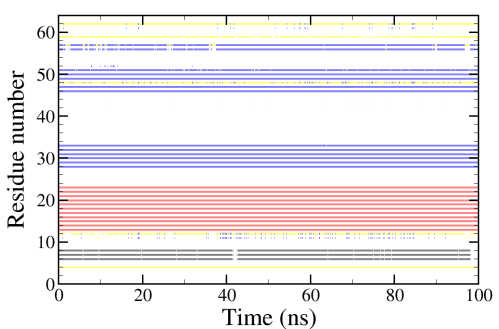

Time (ns)
Full CG / Replica 3

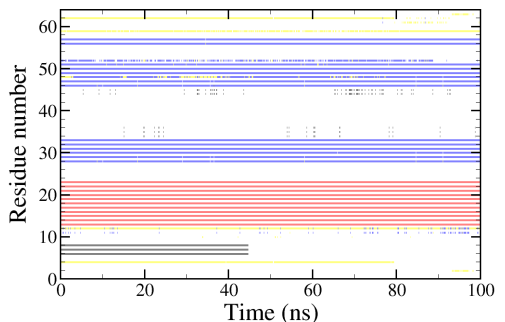

Layer Scheme 2 / Replica 3

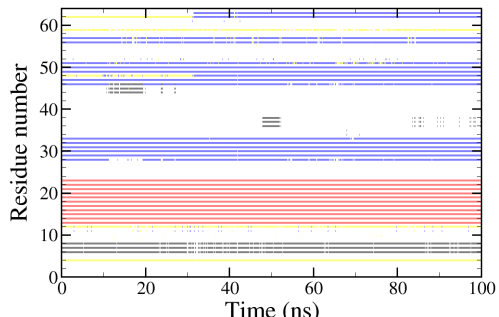

Figure S63: Time series of the secondary structure motifs of the protein in AT water (top), CG water (middle), and AT/CG water with layer scheme 2 (bottom). $\beta$-strands are shown in blue, $\beta$-bridges in yellow, $3_{10}$-helices in black, $\alpha$-helices in red, and $\pi$-helices in green. 


\subsection{Protein 3eye}

Full AT
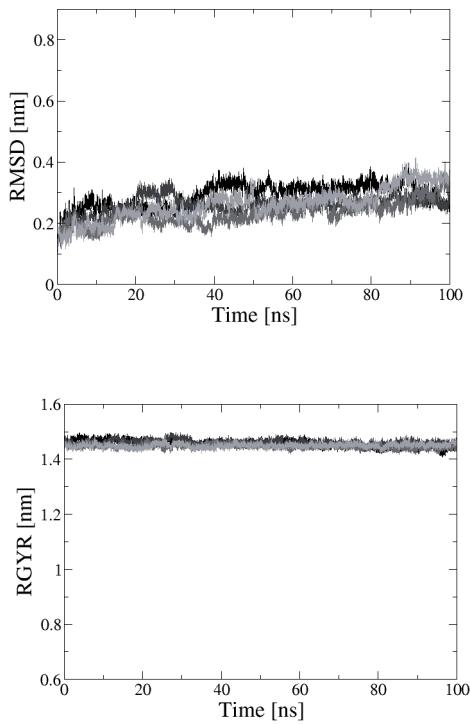

Full CG
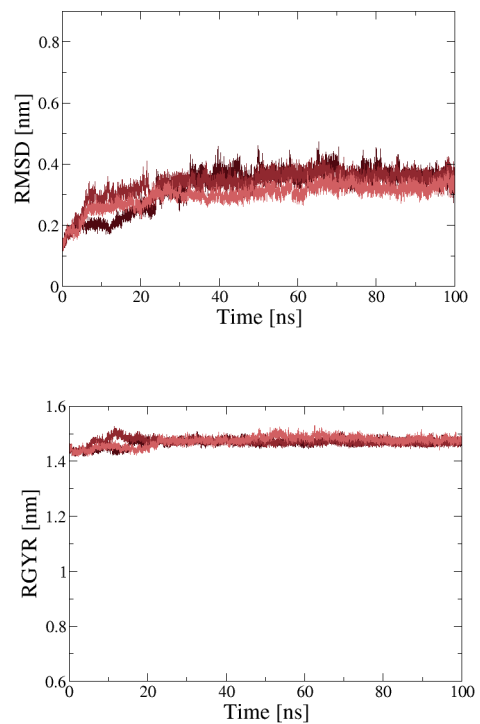

Layer Scheme 2
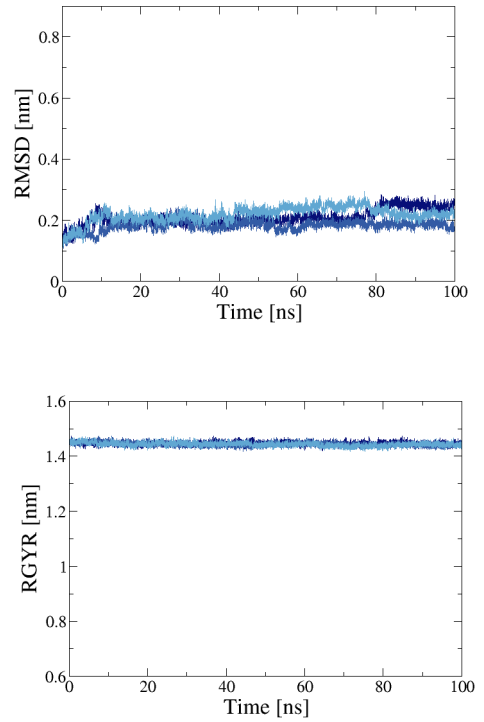

Figure S64: Time series of the backbone atom-positional root-mean-square deviation (RMSD) (top) and the radius of gyration (RGYR) (bottom) of the protein in AT water (black, left), CG water (red, middle), and AT/CG water with layer scheme 2 (blue, right). The replicas are shown in color gradients.
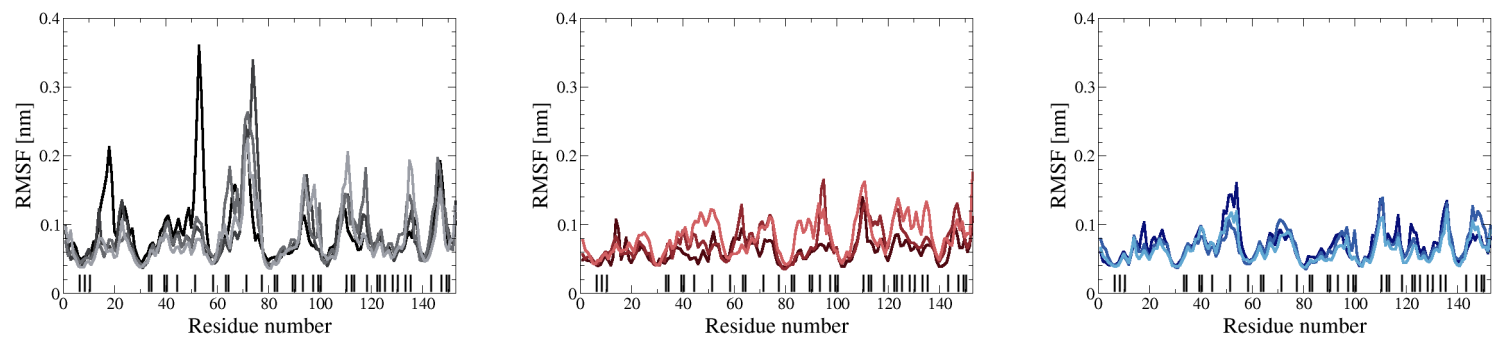

Figure S65: Root-mean-square fluctuation (RMSF) of the $C_{\alpha}$ atoms of the protein in AT water (black, left), CG water (red, middle), and AT/CG water with layer scheme 2 (blue, right). The replicas are shown in color gradients. The positions of the charged residues are indicated with a black dash on the $x$-axis. 
Full AT / Replica 1

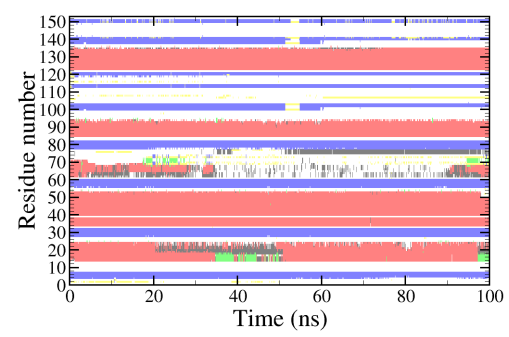

Full AT / Replica 2

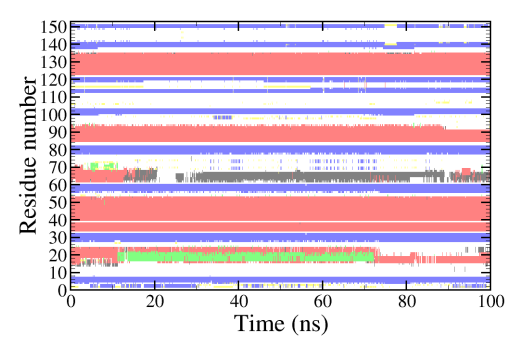

Full AT / Replica 4

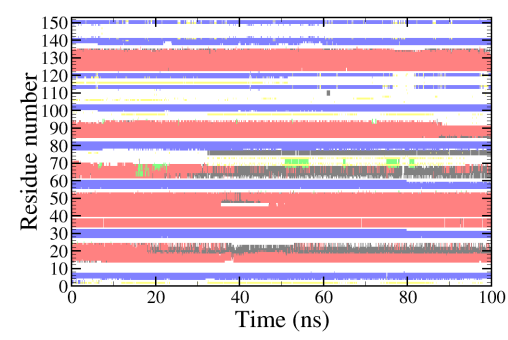

Full CG / Replica 1

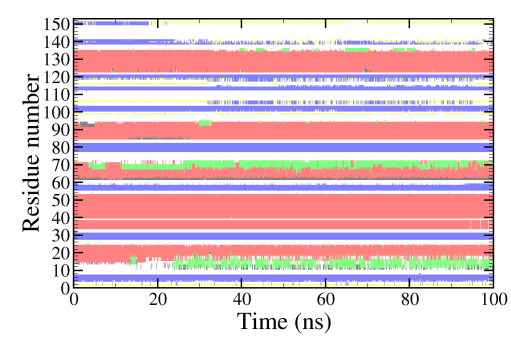

Layer Scheme 2 / Replica 1

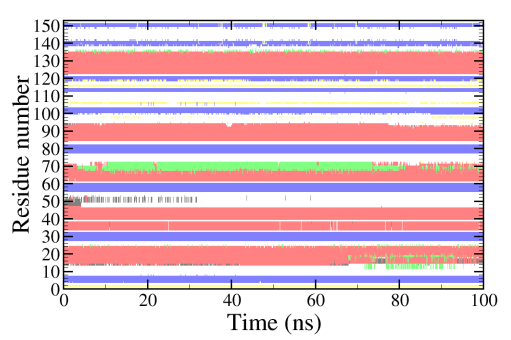

Full CG / Replica 2

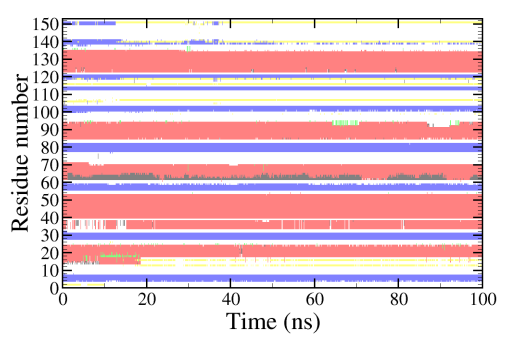

Layer Scheme 2 / Replica 2

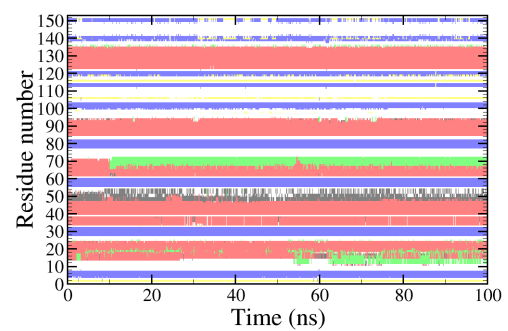

Full AT / Replica 3
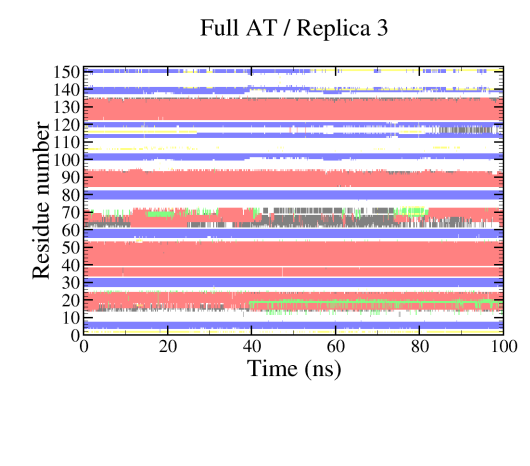


\section{Additional Figures}

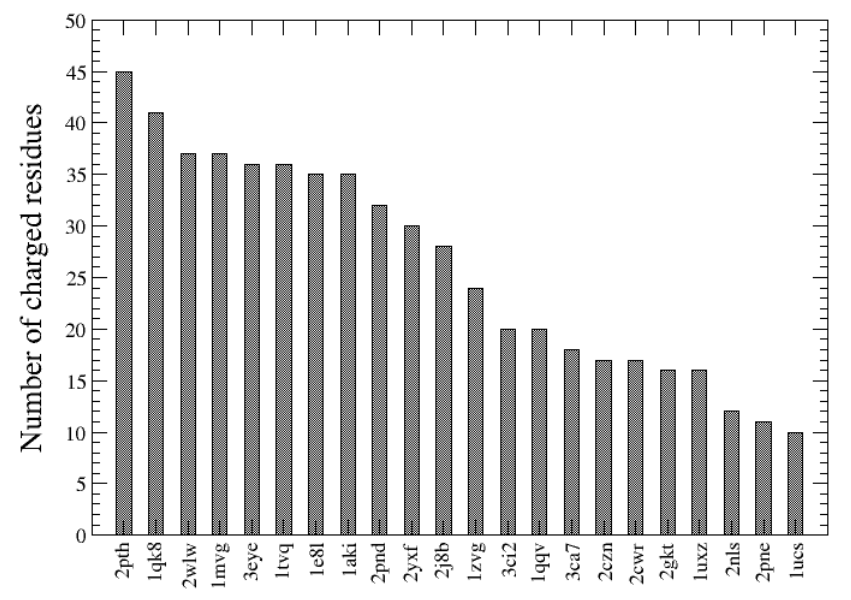

Figure S67: Number of charged residues for the 22 benchmarking proteins in decreasing order. 